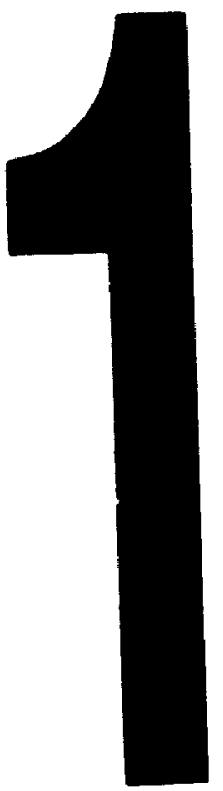

PM-1 312/2 " A" PHOTOGRAPHIC MICROCOPY TARGET NES 1010a ANS1/1SO \#2 EOUIVALENT

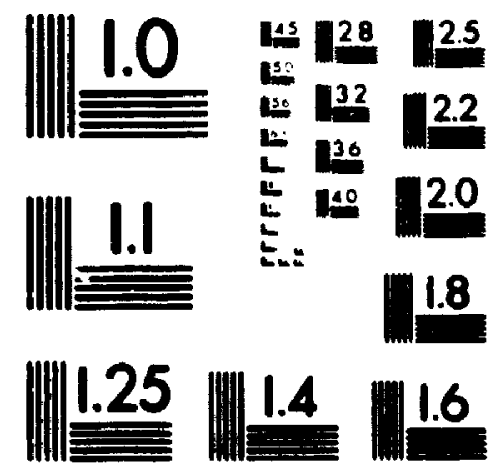

PRECISIONEM RESOLUTION TARCETS 
National Librany

of Canada

Acquisitions and

Bibliographic Services Branch

$3 e 5$ Welungion Street

Oilawa, Oniario

KIA ONA
Bibliothècue nationale

du Canada

Drection des acquisitions et

des services bibliographques

395. ne Wellungton

Otiawa (Ontario)

KIAONA roum vince reterme

Out wo nowre eterenge

NOTICE

AVIS

The quality of this microform is heavily dependent upon the quality of the original thesis submitted for microfilming. Every effort has been made to ensure the highest quality of reproduction possible.

If pages are missing, contact the university which granted the degree.

Some pages may have indistinct print especially if the original pages were typed with a poor typewriter ribbon or if the university sent us an inferior photocopy.
La qualité de cette microforme dépend grandement de la qualité de la thèse soumise au microfilmage. Nous avons tout fait pour assurer une qualité supérieure de reproduction.

S'il manque des pages, veuillez communiquer avec l'université qui a conféré le grade.

La qualité d'impression de certaines pages peut laisser à désirer, surtout si les pages originales ont été dactylographiées à l'aide d'un ruban usé ou si l'université nous a fait parvenir une photocopie de qualité inférieure.

Reproduction in full or in part of this microform is governed by the Canadian Copyright Act, R.S.C. 1970, c. C-30, and subsequent amendments.

La reproduction, même partielle, de cette microforme est soumise a la Loi canadienne sur le droit d'auteur, SRC 1970, c. C-30, et ses amendements subséquents. 


\title{
Neural Network-based CAD for High-Speed Interconnects and Monolithic Inductors
}

\author{
by
}

\author{
Anand Veluswami
}

A thesis submitted to the Faculty of Graduate Studies and Research in partial fulfilment of the requizements for the degree of Master of Engineering

Ottawa-Carleton Institute for Electrical Engineering

Department of Electronics

Carleton University

Ottawa, Ontario, Canada

November 28, 1995

(C) Copyright, 1995

Anand Veluswami 
National Library

of Canada

Acquisitions and

Bibliographic Services Branch

395 Wellington Street

Otiawa. Ontano

K1A ONis
Bibliotheque nationale

du Canada

Direction des acquisitions et

des senvices bibliographiques

395. tue Wettington

Onawa (Ontario)

Var the vare numence

Oute moveremenes

The author has granted an irrevocable non-exclusive licence allowing the National Library of Canada to reproduce, loan, distribute or sell copies of his/her thesis by any means and in any form or format, making this thesis available to interested persons.
L'auteur a accordé une licence irrévocable et non exclusive permettant à la Bibliotheque nationale du Canada de reproduire, prêter, distribuer ou vendre des copies de sa thèse de quelque manière et sous quelque forme que ce soit pour mettre des exemplaires de cette thèse à la disposition des personnes intéressées.

L'auteur conserve la propriété du droit d'auteur qui protège sa thèse. Ni la thèse ni des extraits substantiels de celle-ci ne doivent être imprimés ou autrement reproduits sans son autorisation.
The author retains ownership of the copyright in his/her thesis. Neither the thesis nor substantial extracts from it may be printed or otherwise reproduced without his/her permission.

ISBN $\quad 0-612-08986-X$ 
Nem ANFNTS VELUIWAM!

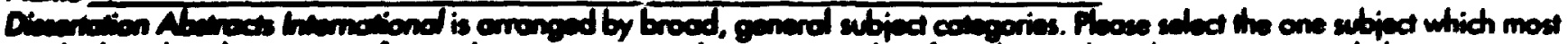

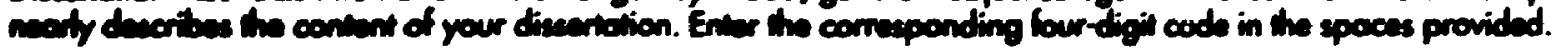

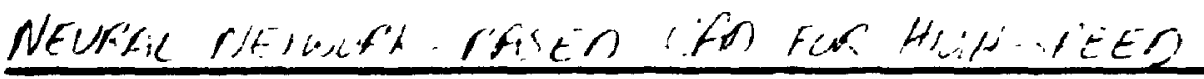

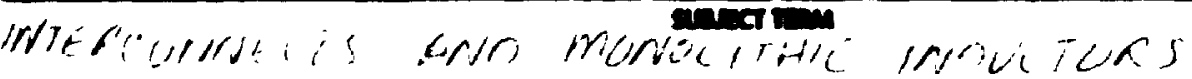

61544 U.M.I

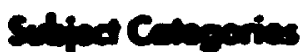

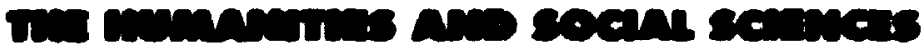
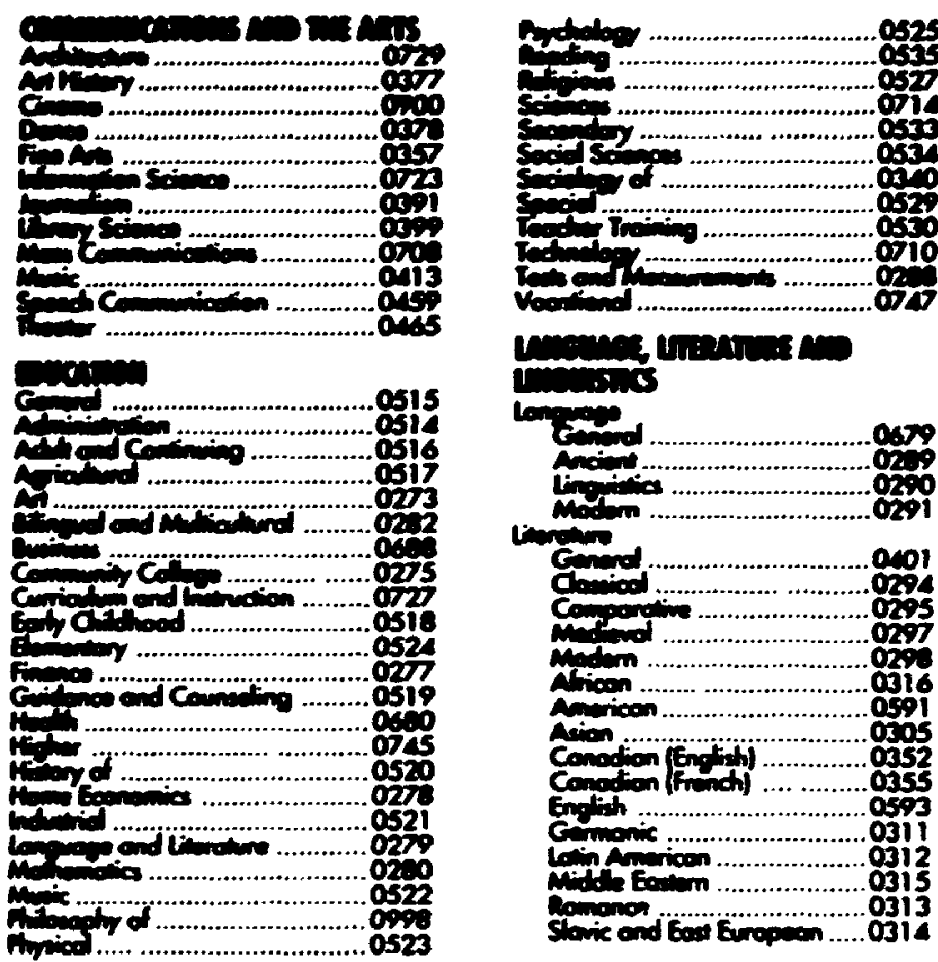

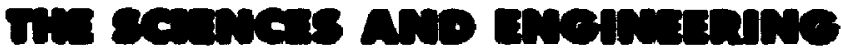

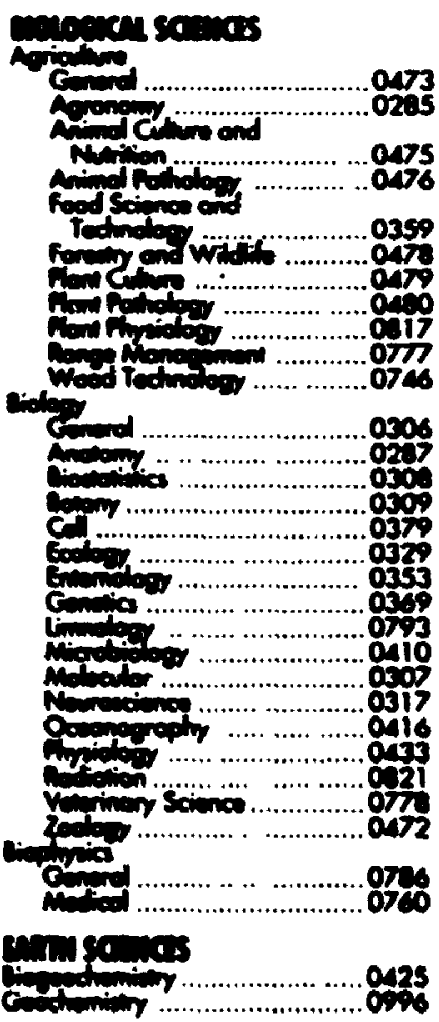

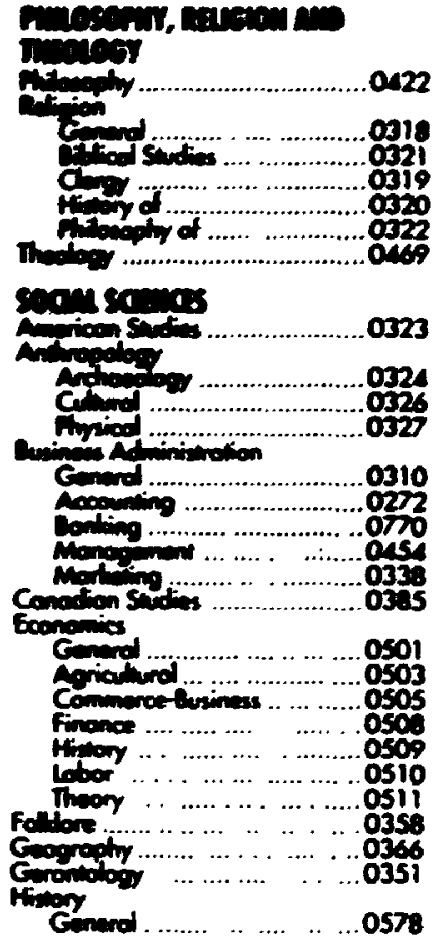

022

0318

03219

0320

(1)

ceren er

nimsente

arisici

Cong

0323

0324

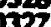

no...................

Gand .........................0310

acting ............................077

Mation ....... .......p25

Compton Shding

Conchice

C.

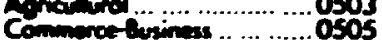

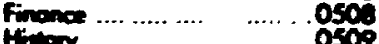

Thony +..............0sil

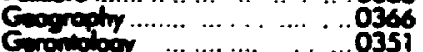

Cinger

0578
Mony ... ............

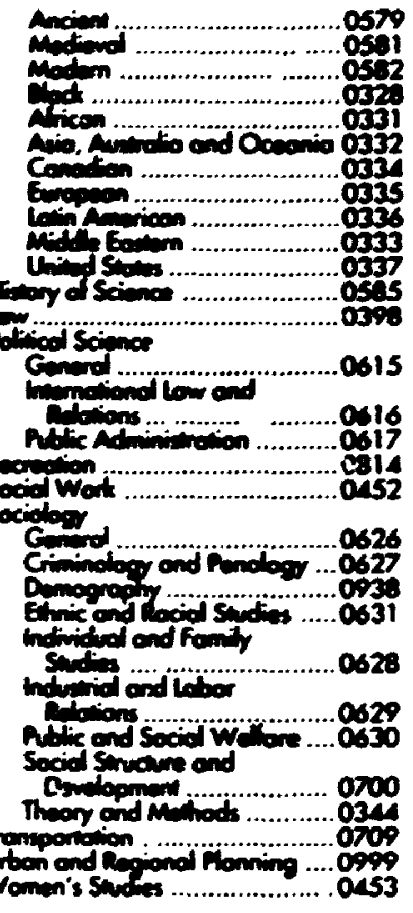

Spench Pothology ..........0460 Toncology. .... …....039.3

rutrensales

pre Scienos:

Ongingy

Genived.

Aretived.

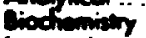

morponis

Nuctor

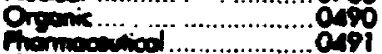

Friced ..............................049

Pocher

noxionion

Menencicas

Prinia

Genwrd ....................opgs

Acoumia

Alromony and

Antroling 060

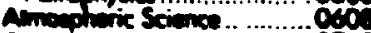

Abmic ... .....................074

Enchonis ond aworicing .....0607

Enuming forides and

Hig E E

078

Hr. ond han .................0759

........

10.......................... O510

Opta............................0752

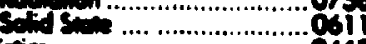

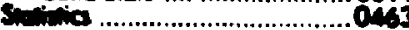

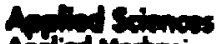

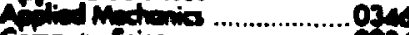

oges

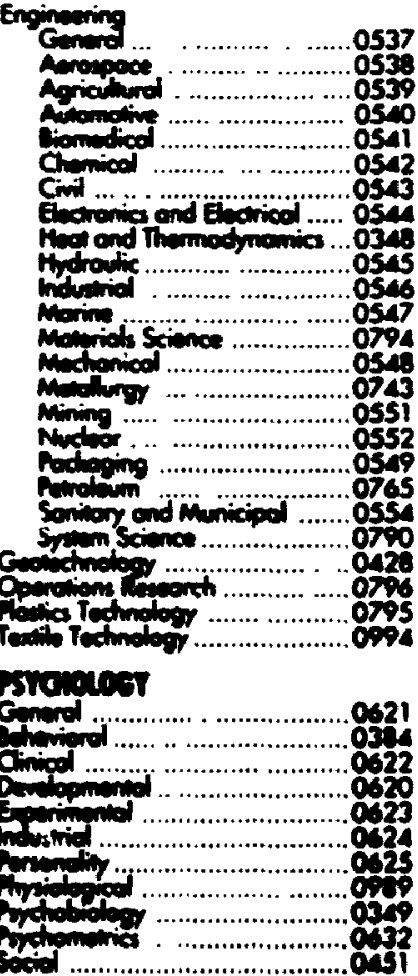


The undersigned recommend to the Faculty of Graduate Studies and Research acceptance of the thesis

\section{Neural Network-based CAD for High-Speed Interconnects and Monolithic Inductors}

submitted by Anand Veluswami

in partial fulfilment of the requirements for the degree of Master of Engineering
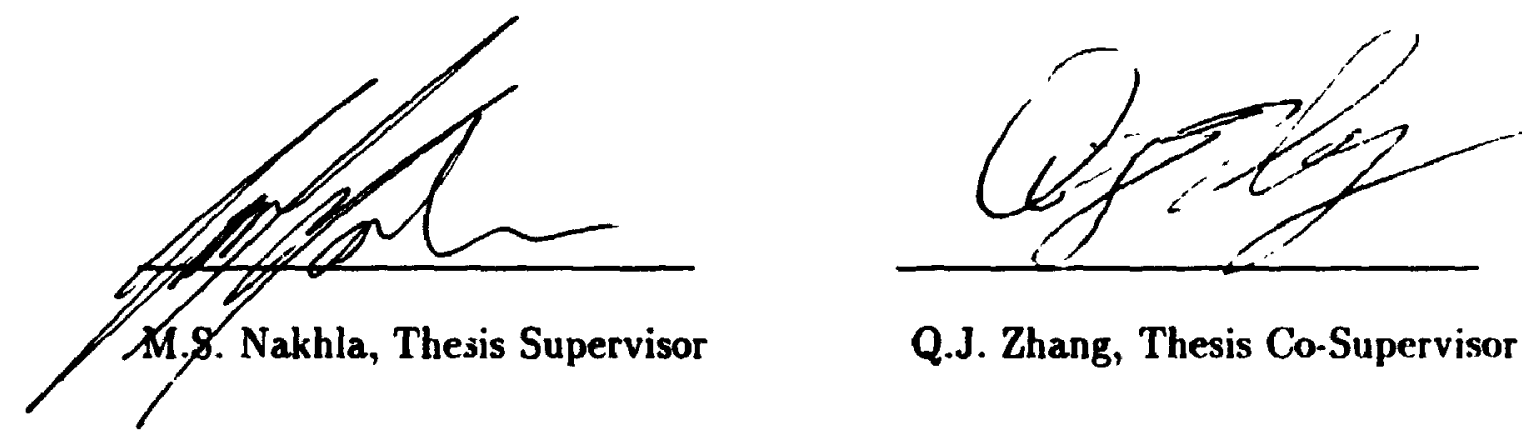

Q.J. Zhang, Thesis Co-Supervisor

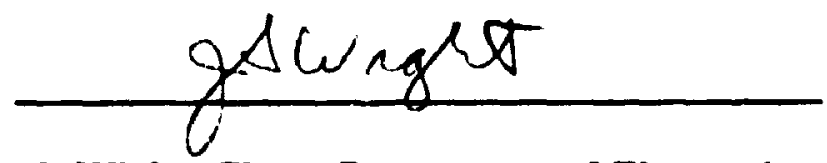

J. Wight, Chair, Department of Electronics 


\section{Abstract}

This thesis investigates the application of artificial neural networks to the computeraided design of high-speed interconnects and monolithic inductors. Traditional techniques used to model passive components in VLSI/RF circuits are based on electromagnetic field simulation and are thus highly demanding on CPU resources. This limits their suitability for use in state-of-the-art design and optimization techniques which are, in general, iterative in nature. Neural networks can be used to map the complex relationships between the physical and electrical parameters of such structures in an efficient yet simple manner. The models, once developed, operate with minimal on-line CPU resources and are thus ideally suited for use in iterative CAD and optimization routines. 


\section{Acknowledgements}

I wish to place on record my sincere gratitude towards my academic supervisor, Mike Nakhla and co-supervisor, Q.J. Zhang, for their continued support, guidance and encouragement throughout my graduate program.

The interconnect-related problems in this thesis have largely been derived from research being done at Physical Design Methods, Bell-Northern Research Ltd., Ottawa. Koko Mihan's several insights in interconnect and signal integrity analysis, and his help in formulating the problems studied have been instrumental, and are much appieciated. Brian Stacey and Greg Wilson, also from Physical Design Methods, BNR, must be also thanked for giving me access to the resources in their division, SALI in particular, and guidance on the usage of SALI.

A significant part of this work has been inspired by the doctoral $r$ iearch of fellow graduate student John Lorg. I am indebted to him for all his insights on the design and characterization of the monolithic inductor, as well as for providing the software: programs I needed to develop neural network models. I also thank him profusely for showing me what dedication and high standards in research at the graduate level are all about.

Carl Mills, B.J. ('94), my thesaurus-friend must also be thanked for his numerous suggestions and input. 


\section{Contents}

1 Introduction 1

1.1 Motivation . . . . . . . . . . . . . . . . 1

1.2 Objectives ..................... 2

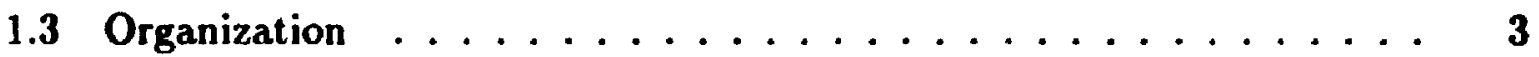

2 High Frequency Analysis: Concepts and Trends 6

2.1 Introduction .................... 6

2.2 The High-Speed Interconnect $\ldots \ldots \ldots \ldots \ldots$

2.2.1 Definition ................... 6

2.2.2 High-Speed Interconnect Analysis $\ldots \ldots \ldots \ldots \ldots$

2.3 Low Run-Time: A Priority in CAD Techniques for Interconnects . . 8 8

2.4 An Overview of Interconnect Techniques $\ldots \ldots \ldots \ldots$

2.4.1 Lumped Parameter Models . . . . . . . . . . . . . . 9

2.4.2 Transmission Line Techniques . . . . . . . . . . 10

2.4.3 Numerical Inversion of Laplace Transforms (NILT) . . . . 11

2.4.4 Asymptotic Waveform Evaluation (AWE) $\ldots \ldots \ldots \ldots$

2.4.5 Modeling the Interconnect at High Frequencies . . . . . . . 12

2.4.6 The Move towards Empirical Relationships . . . . . . . . 15

2.5 CAD for Passive Microwave Components . . . . . . . . . . 17 
2.6 The Monolithic Inductor $\ldots \ldots \ldots \ldots \ldots$

2.7 Modeling the Monolithic Inductor . . . . . . . . . . . . . 19

2.7.1 Electromagnetic Simulation . . . . . . . . . . 20

2.7.2 Physically-based Lumped Element Techniques . . . . . . 20

2.7.3 Using Measured Data . . . . . . . . . . . . . 21

2.8 Fast On-line Modeling in a CAD Environment . . . . . . . . 22

2.8.1 Curve-fit and Closed-Form Models $\ldots \ldots \ldots \ldots \ldots$

2.8.2 Table Look-up Techniques . . . . . . . . . . . . 24

2.8.3 Simple Lumped-Element Representation . . . . . . . 24

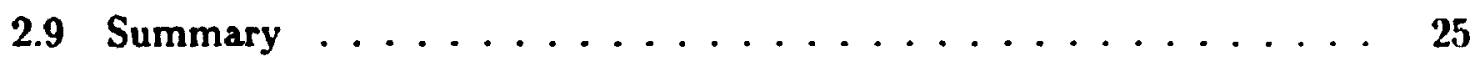

3 Neural Networks: The Concept 26

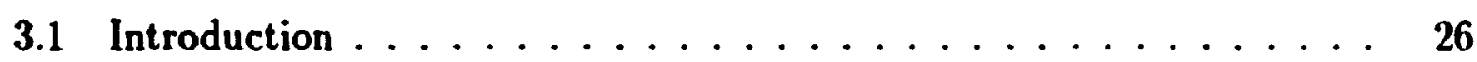

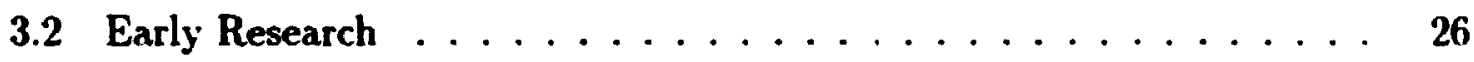

3.2.1 The McCulloch-Pitts Model . . . . . . . . . . 28

3.3 Feed-Forward Neural Networks $\ldots \ldots \ldots \ldots \ldots$

3.3.1 Adaline and The Delta Rule . . . . . . . . . . . 31

3.3.2 Madaline . . . . . . . . . . . . . . . . 33

3.3.3 The Backpropagation Network . . . . . . . . . . . 33

3.4 Feedback Networks $\ldots \ldots \ldots \ldots \ldots \ldots \ldots \ldots$

3.4.1 Hebb's Law . . . . . . . . . . . . . . . 34

3.4.2 Kohonen Self-Organizing Maps $\ldots \ldots \ldots \ldots \ldots \ldots$

3.4.3 Bidirectional Associative Mernory (BAM) $\ldots \ldots \ldots \ldots$

3.4.4 The Spatio-Temporal Network (STN) . . . . . . . . . 39

3.5 Applications of Neural Networks . . . . . . . . . . . . 39 
3.6 In Context: Neural Networks in High Frequency Applications . . . 42

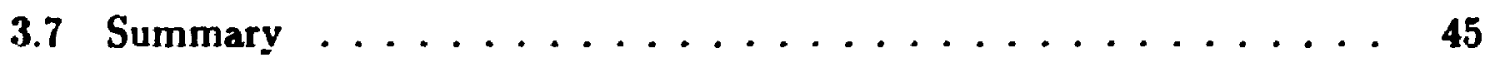

4 The Neural Network Model 46

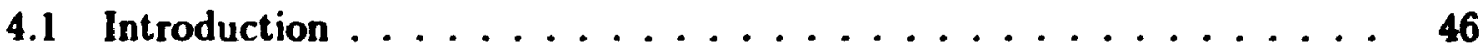

4.2 The Backpropagation Neural Network . . . . . . . . . . . 46

4.3 Construction of The Neural Network Model . . . . . . . . . . . 49

4.3.1 Input/Output Parameters . . . . . . . . . . 49

4.3.2 Training . . . . . . . . . . . . . 52

4.3.3 Data Generation ................. 56

4.3.4 Model Validation . . . . . . . . . . . . . 58

4.4 Summary $\ldots \ldots \ldots \ldots \ldots \ldots \ldots$

5 Neural Networks in Modeling Interconnects 61

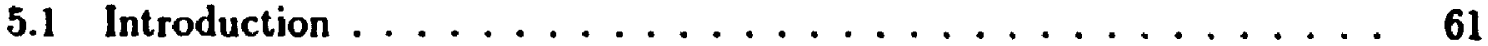

5.2 Example 1: Three Symmetric Microstrips . . . . . . . . . . . . 62

5.2.1 Definition of Input/Output Parameters . . . . . . . . 62

5.2 .2 Construction of the Model . . . . . . . . . . . 63

5.3 Example 2: Two Asymmetric Microstrips . . . . . . . . . . . 68

5.3.1 Identification of Input/Output Parameters . . . . . . 68

5.3.2 The Two Conductor Neural Network Model . . . . . . . 68

5.4 Example 3: An 8-bit Data Bus (Eight Interconnects in Parallel) ... 71

5.4.1 Input/Output Parameters ............. 71

5.4.2 Construction of the Neural Network Model . . . . . . . . 73

5.5 Run-Time Comparison with an EM Field Solver . . . . . . . . . 76

5.6 Discussion of Results . . . . . . . . . . . . . . 77 
5.7 Summary $\ldots \ldots \ldots \ldots \ldots \ldots \ldots \ldots \ldots \ldots$

6 The Interconnect Network Simulator 79

6.1 Introduction $\ldots \ldots \ldots \ldots \ldots \ldots \ldots \ldots \ldots \ldots \ldots \ldots$

6.2 The Interconnect Network Simulator: A Descripiion . . . . . . . . 80

6.2.1 An Interconnect Network: Design Parameters . . . . . . 83

6.2.2 Network Topology: A Design Variable . . . . . . . . . 83

6.3 A Network of Three Interconnects . . . . . . . . . . . . 86

6.3.1 Defining the Input/Output Parameters . . . . . . . 86

6.3.2 Construction of the Model . . . . . . . . . . . 88

6.4 Delay Simulation in a Four Interconnect Network . . . . . . . . 89

6.4.1 The Inputs and Outputs $\ldots \ldots \ldots \ldots \ldots$

6.4.2 Constructing the Four Interconnect Neural Network Model . . 91

6.5 Five Recipient Nodes Connected to a Source . . . . . . . . . . . 92

6.6 Interconnect Networks of Other Sizes . . . . . . . . . . 94

6.6.1 The Cases of A Single Interconnect and Two Interconnects . . 94

6.6.2 Extra Large Networks $\ldots \ldots \ldots \ldots \ldots$

6.7 Performance Comparison with Other Electrical Simulators . . . . . 96

6.8 Discussion of Results . . . . . . . . . . . . . . 97

6.8.1 Evaluation of the Simulator $\ldots \ldots \ldots \ldots 7$

6.8.2 The On-line/Off-line Time Trade-off $\ldots \ldots \ldots \ldots . \ldots 98$

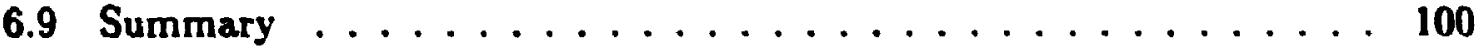

7 Modeling the On-Chip Spiral Inductor 101

7.1 Introduction . . . . . . . . . . . . . 101

7.2 The On-Chip Spiral Inductor Mortel . . . . . . . . . . . 101 
7.2.1 Definition of Input/Output Parameters . . . . . . . . 102

7.2.2 Construction of the Model . . . . . . . . . . . . . 102

7.2.3 Performance Comparison with Existing Techniques . . . . 105

7.3 Applications of the Model $\ldots \ldots \ldots \ldots 7$

7.3.1 On-line Simulation of S-parameters . . . . . . . . 107

7.3.2 Inductor Self-Resonant Frequency Analysis . . . . . . . . 107

7.3.3 Q-Factor Analysis . . . . . . . . . . . . 111

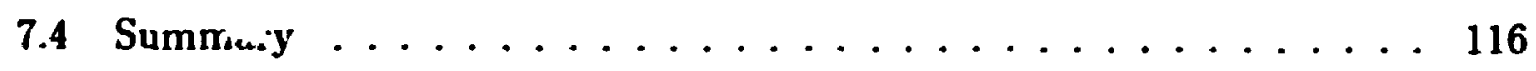

8 Summary and Conclusions $\quad 117$

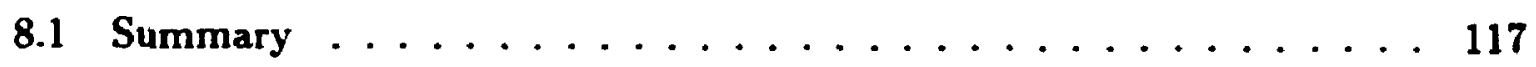

8.2 Neural Network Models in Perspective . . . . . . . . . . 119

8.2 .1 Advantages . . . . . . . . . . . . 119

8.2 .2 Disadvantages . . . . . . . . . . . 120

8.3 Performance Evaluation Against Existing Techniques . . . . . . 121

8.3.1 Electromagnetic-based Simulation . . . . . . . . . . . 121

8.3.2 Table Look-up Models . . . . . . . . . . . . . . . . 122

8.3.3 Closed-Form Equations and Curve-Fit Techniques . . . . . . 123

8.3.4 Lumped-Element Equivalents . . . . . . . . . . . . 124

8.4 Applying the Neural Network Technique . . . . . . . . . . . 125

8.5 Suggestions for Further Research . . . . . . . . . . . . 126 


\section{List of Tables}

5.1 Input parameters and their range (Example 1$) \ldots \ldots \ldots \ldots 2$

5.2 Features of the neural network for the three symmetric multi-conductor $\operatorname{model} \ldots \ldots \ldots \ldots \ldots \ldots \ldots \ldots \ldots \ldots$

5.3 Features of the neural network model for Example 2 . . . . . . 70

5.4 Input parameters and their range for the 8 -bit data bus $\ldots \ldots \ldots 71$

5.5 Features of neural network model for Example $3 \ldots \ldots \ldots$

5.6 Comparison of CPU time requirements for neural network model and

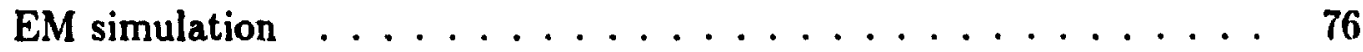

6.1 Number of possible configurations for a given number of interconnects 86

6.2 Range of input parameters to the three interconnect network model . 88

6.3 Features of three interconnect network model $\ldots \ldots \ldots \ldots$

6.4 Range of input parameters for the four interconnect network model . 91

6.5 Features of neural network model (four interconnects) . . . . . . 92

6.6 Features of neural network model for five interconnects . . . . . . 94

6.7 Run-time for simulation using the neural network model, NILT and

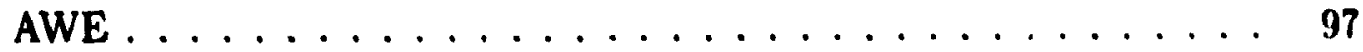

7.1 Input parameters for the inductor model and their range $\ldots \ldots .104$

7.2 Features of the neural network model $\ldots \ldots \ldots \ldots \ldots$ 
7.3 Comparison of on-line simulation run-time . . . . . . . . . 105

7.4 Geometries and self-resonant frequencies of inductors having a lowfrequency inductance of $15 n \mathrm{nH} \ldots \ldots 109$ 


\section{List of Figures}

2.1 A typical CAD/optimization environment $\ldots \ldots \ldots \ldots \ldots$

3.1 The McCulloch-Pitts model $\ldots \ldots \ldots \ldots \ldots \ldots$

3.2 Different kinds of transfer functions $\ldots \ldots \ldots \ldots \ldots \ldots$

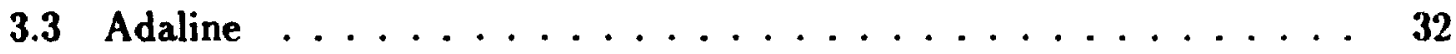

3.4 The Kohonen Self-Organizing Network. Feed-forward and feedback connections are shown, the feedback only indicated for the centre neuron of the output layer $\ldots \ldots \ldots \ldots \ldots \ldots \ldots \ldots$

3.5 The Bidirectional Associative Memory (BAM) structure $\ldots \ldots . .38$

3.6 A neural network-based CAD environment $\ldots \ldots \ldots \ldots \ldots$

4.1 Structure of the Neural Network Model . . . . . . . . . . . 48

4.2 General structure of the model to be developed $\ldots \ldots \ldots \ldots$

4.3 Algorithm used for generation of training and test data . . . . . 57

5.1 Cross-section of three microstrip lines $\ldots \ldots \ldots \ldots \ldots \ldots$

5.2 Average training error as training proceeded for Example $1 \ldots \ldots 6$

5.3 Test error for 100 random configurations for the three conductor model 66

5.4 As a function of separation, parameter $C_{13} \ldots \ldots \ldots \ldots 7$

5.5 Two asymmetric microstrip lines $\ldots \ldots \ldots \ldots \ldots \ldots$ 
5.6 Test error for 100 random inputs from the test set for Example 2 . . $\quad 69$

5.7 8-bit bus on a PCB, showing region of high crosstalk and signal noise 72

5.8 Cross-section of the 8 -bit digital bus ............. 72

5.9 Test error over 100 random inputs for the 8-bit bus model . . . . . 74

5.10 The values of mutual capacitances between the conductors of a given configuration of the 8 -bit bus $\ldots \ldots \ldots \ldots$

6.1 A typical interconnect network with IC pins connected by several interconnects $\ldots \ldots \ldots \ldots \ldots \ldots$

6.2 Electrical equivalent of interconnect configuration, showing the outputs

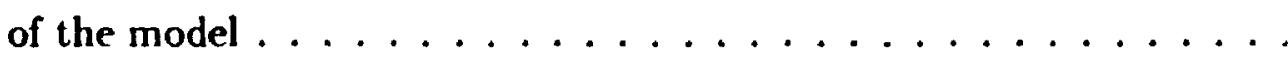

6.3 Possible network configurations for four nodes. The values of the variables defining the topology are also given ............

6.4 Electrical representation of a three interconnect network . . . . . . .

6.5 Error in 100 random test samples of the three interconnect network

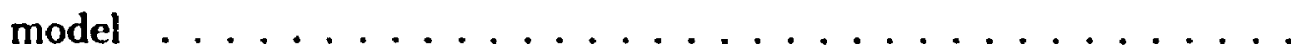

6.6 Error for 100 random circuit configurations for the four interconnect

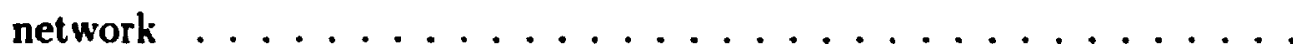

6.7 Error for 100 random circuit configurations for the five interconnect network

7.1 The square spiral inductor ................. 103

7.2 Test error in S-parameter simulation for 100 random spiral inductor

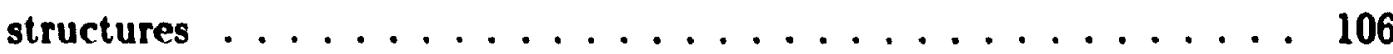

7.3 S-Parameters for a spiral inductor having 8 turns . . . . . . . 108

7.4 Inductance as a function of frequency for spirals of different sizes . . . 110 
7.5 Self-resonant frequency versus physical dimensions for a fixed number of turns ....................... 112

$7.6 \mathrm{Q}$-factor surface at $909 \mathrm{MHz} \ldots \ldots \ldots \ldots \ldots \ldots$

7.7 Q-factor surface at $1.4 \mathrm{GHz} \ldots \ldots \ldots \ldots \ldots \ldots \ldots$

7.8 Q-factor surface at $1.9 \mathrm{GHz} \ldots \ldots \ldots \ldots \ldots \ldots \ldots$ 


\title{
List of Symbols
}

\author{
b bias vector of the hidden layer \\ C per-unit-length Capacitance matrix \\ $C_{i} \quad$ termination capacitance of $i$ th interconnect \\ c bias vector of output layer \\ $E_{a v} \quad$ batch-mode backpropagation error \\ $e_{j} \quad$ edge variable (defining topology) \\ $\mathcal{F}$ objective mapping function \\ G per-unit-length conductance matrix \\ $h \quad$ height of dielectric layer \\ $I(t)$ external inputs in Grossberg learning \\ L per-unit-length inductance matrix \\ $L \quad$ outer dimension of spiral inductor \\ $l_{i} \quad$ length of the $i$ th interconnect \\ $m \quad$ size of input vector $\mathbf{X}$ \\ $N$ number of turns in spiral inductor \\ $n$ size of output vector $Y$ \\ $p$ number of neurons in hidden layer \\ $Q \quad$ size of training/test set
}


xvi

$\mathbf{R}$ per-unit-length resistance matrix

$\boldsymbol{R}_{i} \quad$ termination resistance of $i$ th interconnect

$R$, resistance of source (driver)

$r_{i} \quad$ input rise time

S S -parameters of spiral inductor

$\mathcal{S} \quad$ size of neural network model

$s \quad$ separation between adjacent conductors

$t$ thickness of conductor

$U() \quad$ function controlling the learning in Kohonen networks

$V_{p} \quad$ peak voltage of signal

$v$ weight vector of the hidden layer

W width of microstrip

weight vector of input layer

$\mathbf{X}$ input to model

$\mathbf{Y} \quad$ output to model

$z \quad$ output of an individual neuron

$\alpha \quad$ momentum in training process

$\delta() \quad$ local gradients of neurons

$\epsilon_{q} \quad$ rms error of the $q$ th sample

$\gamma \quad$ the linear weighted combination of the inputs

$\zeta \quad$ the linear weighted combinations of the hidden layer outputs

$\eta \quad$ learning rate parameter

$T_{i} \quad$ propagation delay at $i$ th interconnect

$\omega \quad$ frequency of operation 
xvii

\title{
List of Acronyms and Abbreviations
}

\author{
ADALINE Adaptive Linerer Element \\ ANN \\ Artificial Neural Networks \\ AWE \\ Asymptotic Waveform Evaluation \\ BAM \\ Bidirectional Associative Memory \\ CAD \\ Computer-Aided Design \\ CPU \\ Central Processing Unit, CPU resources connoting com- \\ puter resources
}

CFH

Complex Frequency Hopping

ECG

Electro-cardiogram

EEG

Electro-encephalogram

FDM

Finite Difference Method

FEM

Finite Element Method

GaAs

Gallium Arsenide

LVQ

Learning Vector Quantization

MADALINE

Many Adaptive Linear Elements

MCM

Multi-chip Module

MIC

Microwave Integrated Circuit

MLP

Multi-layer Perceptron 
MMIC

MOM

MOSFET

MRI

NILT

PCB

PCS

RF

sms

SALI

SRAM

STN

TEM

VLSI
Monolithic Microwave Integrated Circuit

Method of Moments

Metal-On-Semiconductor Field-Effect Transistor

Magnetic Resonance Imaging

Numerical Inversion of Laplace Transforms

Printed Circuit Board

Personal Communication System

Radio Frequency

root mean squared

Structural Analysis of Lossy Interconnect [93]

Static Random Access Memory

Spatio-temporal Network

Transverse Electromagnetic, as in TEM mode of propagation

Very Large Scale Integration 


\section{Chapter 1}

\section{Introduction}

\subsection{Motivation}

High frequency electronic circuit design is experiencing tremendous growth and development both on the research and industrial fronts, with levels of integration and operating frequencies increasing every day. This growth has largely been possible due to parallel advances in computing resources and the availability of advanced computeraided design (CAD) tools which enable extensive design, analysis and optimization, and can be sustained only if the domain of CAD and simulation tools makes similar advances.

Modeling and simulation at high frequencies is significantly more complex than at low frequencies due to the fact that as the physical dimensions of a circuit approach the wavelength of the operating signal (signals typically being in the microwave or millimeter wave region), distributed parameter models must be used to accurately characterize its performance. These models are as such highly involved, as they are based on numerically solving equations like Maxwell's Equations or the transmission line equations. 
A classical example of a complex simulation task of this kind is the analysis of interconnects in a high-speed VLSI circuit. When carrying signals of high speed, interconnects begin to load the circuit elements, exhibit mutual inductive and capacitive coupling, and cause crosstalk between signals they are carrying. They must he accurately characterized during design in order that critical operational errors be obviated.

Another such task is in the design of passive components used in analog RF and microwave integrated circuits (MICs), such as the monolithic inductor. Circuits for MICs must be designed and analyzed meticulously, especially monolithic circuits (MMICs), wh e high yield and mass manufacturability are key objectives.

Both these tasks require electrical and electromagnetic analysis, or both, to be performed for accurate characterization, which are computationally very expensive. The long run-times pose even more of a difficulty in view of the large number of components in any typical circuit, especially in the case of interconnects, as well the fact that most currently employed CAD and optimization are based on highly iterative routines. Thus it is imperative to have fast models which can provide on-line speed for incorporation into such routines. There are several existing methods of providing on-line simulation speed, such as by using look-up table models, polynomial curve-fit and closed-form models, or simple lumped circuit representations.

\subsection{Objectives}

This thesis investigates an alternative approach to the on-line analysis and characterization of high-speed interconnects and monolithic inductors. Artificial neural networks, a powerful artificial intelligence technique, have been applied recently to 
several problems in the physical sciences and engineering, as a simple yet robust tool capable of learning the complex relationships that exist in the information presented to it. Neural network models for high frequency structures, such as interconnects and monolithic inductors, can be used to characterize them accurately and efficiently.

A general neural network methodology applicable to a wide range of high frequency circuit problems is formulated, and applied to specific problems of interest. Models for the estimation of the frequency-dependent parameters of multi-conductor microstrip lines are developed. An interconnect network simulator consisting of neural network models that can simulate propagation delay is constructed. Thirdly, the $S$-parameters of the monolithic inductor are modeled based on its physical and geometric parameters. After each model is constructed, it is compared with existing techniques in terms of $\mathrm{CPU}$ resource requirements.

The models developed provide a basis to evaluate the neural network approach in computer-aided design. After comparing the neural network technique to existing modeling and simulation techniques, general guidelines for applying the neural network technique to CAD-related problems are given.

\subsection{Organization}

Chapter 2 gives a conceptual background to computer-aided design techniques for high-speed VLSI interconnects and passive microwave structures, in particular the monolithic inductor. The two broad classes of problems in interconnect analysis, namely interconnect modeling and interconnect network simulation, are elucidated. The desire for a high computational speed in models used in CAD and optimization routines is described. A survey of current modeling and 
simulation techniques is provided to give a background to the problem, and to indicate the extent of research efforts in this area.

Chapter 3 provides an overview of neural network theory, and the basic types of neural networks. Though the applications in this thesis require only one type of neural network, a general idea of different types of neural networks can give better insight into the learning principles shared by all neural network architectures. A discussion of the applications of neural networks in different areas, and of neural networks in the context of CAD and optimization applications is given.

Chapter 4 describes the neural network model, and a method of formulation of CAD-related problems into neural network models. The backpropagation algorithm used to train neural networks is discussed. Methods of validating the model, and of data generation from existing simulation approaches are presented.

Chapter 5 describes the application of neural networks to problems involving the modeling of the per-unit length parameters of interconnects. Models are developed to obtain these electrical parameters, where traditional approaches use electromagnetic analysis. The models constructed are compared to existing techniques.

Chapter 6 gives examples of neural network models for simulating networks of interconnects in high-speed digital circuits. An interconnect network simulator which consists of a group of neural network models that simulate interconnect networks of different sizes is discussed. A method of expressing network topol- 
ogy as a variable is described. Here again, comparisons with existing techniques are made.

Chapter 7 demonstrates the modeling of the monolithic inductor with neural networks. The spiral inductor under study derives its physical and geometric specifications from wireless Personal Communication System (PCS) applications in a silicon-based production technology. The strength of the neural network approach to circuit design and analysis is shown by applying the model to inductor analysis and characterization problems.

Chapter 8 summarizes the thesis. The advantages and disadvantages of the neural network approach are studied both in general, and then over existing simulation techniques individually. Based on the results obtained in the previous chapters, general guidelines as to which problems would be best suited for neural networks are provided. Suggestions for further research are made. 


\section{Chapter 2}

\section{High Frequency Analysis: Concepts and Trends}

\subsection{Introduction}

In this chapter, a literature survey of existing techniques for the computer-aided design and analysis of interconnects and passive microwave structures is presented. Interconnect techniques are classified as modeling and simulation techniques, and each is described separately. Methods available for modeling and characterizing passive microwave structures, specifically the monolithic inductor, are then discussed. The methods commonly employed to simplify on-line CPU run-time requirements, such as curve-fitting techniques and table look-up techniques are briefly described.

\subsection{The High-Speed Interconnect}

\subsubsection{Deflnition}

A high-speed interconnect can be defined as an interconnection between discrete components of a digital circuit which cannot be taken to be an ideal conductive 
medium but rather must be considered as a circuit component that influences circuit performance $[1, \mathrm{pg} .5]$.

As signal frequency increases, a metallic interconnect in a VLSI circuit does not ideally transmit a signal from its source to its destination, but introduces many characteristic effects of its own. Package mismatch, signal propagation delay, line losses, crosstalk, ground noise, and ringing are among these effects [2]-[6],[16]. These effects must be accounted for by modeling the interconnects as individual components themselves with models which would reflect these effects on circuit performance.

\subsubsection{High-Speed Interconnect Analysis}

Analysis of the high-speed interconnect involves modeling its electrical characteristics, as well as simulating the effects it may have on circuit performance. At high frequencies, the physical dimensions of the interconnect become comparable to the wavelength of the signal, and hence lumped parameter models cease to be accurate. The use of more complex simulation techniques, such as distributed parameter models, or full electromagnetic field analysis, is required.

High-speed interconnect analysis can be classified for simplicity into two broad divisions, interconnect modeling and interconnect simulation:

Modeling encompasses problems where the outputs are intrinsic to the interconnects, based on their own physical and geometrical properties, and independent of other circuit components. Typical examples are the RLCG parameters and the Sparameters. In such cases, electromagnetic field analysis of the interconnect structure is performed, based only on its physical dimensions and characteristics. It is independent of the rest of the components to which it is connected. 
Simulation involves the estimation of circuit level issues such as the signal propagation delay or crosstalk in an interconnect or network of interconnects on a Printed Circuit Board (PCB). The interconnects here are considered part of a larger circuit, with a source and terminations. Here, electrical network analysis must be performed to obtain the required parameters. Performance-driven layout problems require such simulation techniques in order to optimize circuit layouts.

Techniques for modeling and simulation of high-speed interconnects are described in more detail in Section 2.4 .

\subsection{Low Run-Time: A Priority in CAD Techniques for Interconnects}

Along with accuracy, a low run-time is one of the most desirable properties of any CAD technique, interconnect techniques in particular. This is because of the excessive use of the interconnect techniques in these routines. The repetitive use of interconnect models is attributed to the following two factors.

Firstly, any given circuit consists of a large number of interconnects, their number often far outnumbering that of the active devices and components. Every active or discrete component on a PCB must have at least one interconnect carrying a signal to its input, and an interconnection network carrying its output to other components. Several of these, especially the critical paths in the circuit (e.g. those carrying the clock signals), are all potential factors contributing to crosstalk, propagation delay and other effects, and are thus areas of focus during circuit design, layout and optimization.

Secondly, current circuit optimization techniques are by and large ilerative in 
nature. An optimization objective function is defined, and the circuit and its components are configured and reconfigured in as many different ways as possible, attempting to cover exhaustively yet systematically the entire range of possible variations. The configuration which best satisfies the predefined optimization objective function without violating any of the design specifications and constraints is chosen to be the optimal solution. Currently employed optimization techniques such as steepest descent, Newton-Raphson, least pth optimization, minimax, etc. are all highly iterative in nature $[7,8]$.

The problem of PCB layout optimization, wiring, and physical package design, which are direct applications of high-speed interconnect network analysis, are discrete optimization problems of large dimension. These problems are often solved by statistical approaches such as simulated annealing and Monte Carlo techniques such as those described in [9]-[11], which are also highly iterative in nature.

A typical example of an optimizer is Optimization Systems Associates' OSA90 [12], which can perform most of the above stated optimization and statistical analysis operations.

\subsection{An Overview of Interconnect Techniques}

\subsubsection{Lumped Parameter Models}

The first attempts at modeling interconnects were by using lumped parameter techniques, such as the RC tree. The simple RC tree consists of a series resistor with a grounded capacitor. A typical example is that proposed by Rubinstein and Penfield in 1984 [13]. This can be used to calculate the "Elmore delay" in an interconnect, which is the first moment of its impulse response [14]. 
The RC tree is capable of handling very simple cases with step responses at relatively low frequencies. However, it is a simple one-pole approximation, and is thus incapable of accounting for inductive effects of interconnects, such as ringing. An additional drawback of lumped-element techniques is the large computational time required. The run-time of Spice and similar circuit solvers depends on the size of the network, and increases with the addition of each passive element of the RC/RLC trees.

Recent attempts at simplifying the CPU time required for simulation of RLC tree networks, such as the two-pole approximation method by Zhou et al. [15], can reduce computational run-times, but these are based on simplification and approximation of the original structure, and thus compromise on accuracy in the process of providing speed.

\subsubsection{Transmission Line Techniques}

At relatively higher frequencies, lumped parameter models cannot adequately represent interconnect phenomena. Fully distributed parameter models, such as the transmission line model, must be employed for high-speed interconnect analysis. In these approaches, parameters such as the per-unit-length resistance, capacitance, inductance and conductance are required. If there is more than one interconnect, modeling the mutual effects between them requires the RLCG matrices describing their coupling. They assume quasi-TEM wave propagation, and hence are valid for the range of frequencies where the assumption that the physical dimensions of the interconnect structure are much smaller than the wavelengths of the signals propagating holds. Several publications exist in the literature describing these transmission line models, such as $[4,5,17]$. Chiprout, in his doctoral thesis, also provides an account 
of the development of research in transmission line techniques for interconnects [18, pp.11-16].

As such, transmission line models are inherently slow. The problem is compounded when they have non-linear terminations, in which case both time-domain analysis and frequency-domain analysis must be combined [1]. The problem of the CPU intensity of high-speed interconnect techniques bas led to the development of several techniques aimed at reducing run-times, such as NILT and AWE, described in the following sections.

\subsubsection{Numerical Inversion of Laplace Transforms (NILT)}

An important technique used in time-domain analysis is the Numerical Inversion of Laplace Transforms (NILT). NILT has been applied to interconnect analysis to incorporate transmission line models, as well as to perform time domain analysis of interconnects with non-linear terminations [19].

NILT is based on the Padé approximation of the exponential function to a rational function. The coefficients of the rational function are computed beforehand and stored in tables. These is used to calculate the time-domain response of a network from the Laplace domain. A detailed description of the technique can be had from $[7,19]$.

The NILT technique is popular because it not only reduces the CPU requirements for solving the circuit equations, but it allows time-domain simulation to commence at any time instant. In other words, it is not required to start at $t=0$ to obtain the time-domain response, as is required in most other techniques. It is a widely used tool in several industrial simulation environments [9], and is the method chosen to generate the data required for the PCB applications in this thesis. 


\subsubsection{Asymptotic Waveform Evaluation (AWE)}

Another technique which has generated much interest recently is Asymptotic Waveform Evaluation (AWE). AWE has been used to perform analysis of interconnects using moment-matching techniques based or Padé approximation $[2,20,21]$.

AWE is based on the approximation of the Taylor series response of a network into a simpler, low-order transfer function using rational Padé approximation. The original network, which could consist of distributed transmission line models, is thus represented by a rational expression, the solving of which is much less CPU intensive than the original equations defining the network. A mathematical description of AWE can be had from any of the above references.

The AWE technique has also been extended to interconnects with non-linear terminations [22]. Improvements on the basic technique, such as Complex Frequency Hopping (CFH) [23], have greatly enhanced its capability to account for transmission line effects such as ringing and crosstalk, which were handled inadequately by the original technique. Complex Frequency Hopping approximates the network response after mılti-point expansion of its Taylor series. This enables a more complete search for dominant network poles.

Very recently AWE and the technique of Complex Frequency Hopping have been extended to frequency-dependent parameter analysis of interconnects [24], and to full-wave analysis [25].

\subsubsection{Modeling the Interconnect at High Frequencies}

In the transmission line models described above, the per-unit resistance, inductance, capacitance, and conductance (RLCG) matrices are required. The transmission line 
equations for multi-conductor lines, as described in [26], can be expressed as

$$
\begin{gathered}
\frac{\partial}{\partial z} \mathbf{V}(z, t)=-\mathbf{R I}(z, t)-\mathbf{L} \frac{\partial}{\partial t} \mathrm{I}(z, t) \\
\frac{\partial}{\partial z} \mathbf{I}(z, t)=-\mathbf{G V}(z, t)-\mathbf{C} \frac{\partial}{\partial t} \mathbf{V}(z, t)
\end{gathered}
$$

At relatively lower frequencies, the RLCG parameters are assumed to be constant, but as the frequency of operation increases, the signal wavelength becomes comparable to the physical dimensions of the structure, this assumption ceases to be valid, and they must be modeled independently before the transmission line equations can be used [26].

In order to obtain approximation-free results, full-wave analysis must be performed on the interconnect structure. This takes into account all possible modes of wave propagation and all boundary conditions in the structure. But as this operation is complex and demanding on CPU resources, the TEM approximation is made. This approximation presupposes only transverse electromagnetic wave propagation, assuming that the electromagnetic field components along the $z$-axis do not exist. In the case of a lossy interconnect, there is a finite value of electrical resistance along it, which means there is voltage, and thus an electric field in the direction of the 2-axis. This invalidates the TEM assumption. Similarly, an inhomogenous surrounding medium cannot support TEM wave propagation. However, if these effects are small, the TEM assumption can still be made during analysis and render results of tolerable accuracy. This is often referred to as quasi-TEM approximation, and is widely employed in modeling interconnects for their RLCG parameters. Paul, in his book [26], explains a method of evaluating the RLCG parameters based on the 
quasi-TEM assumption.

Modeling the $\mathrm{L}$ and $\mathrm{C}$ parameters of an interconnect structure involves solving the Laplace Equation under static conditions in the two-dimensional transverse plane. In most practical cases, such as on a PCB, the surrounding medium is lossy and hence the method used in calculating the $\mathrm{L}$ and $\mathrm{C}$ parameters must be modified to incorporate these losses. These are represented by the dielectric loss tangent, which is generally a function of frequency. Also, in a lossy interconnect the non-uniform current distribution in the conductor's cross-section necessitates a modification in the values of the L parameters calculated for the lossless case.

The $\mathbf{R}$ matrix is calculated based on the current density and distribution in the cross-section of the interconnect structure, which is governed by the Helmholtz equation. Calculating the $\mathbf{R}$ parameters is further complicated the need to account for the skin effect. At higher frequencies, the flow of current is concentrated away from the centre of the conductor and more towards its surface. In the case of conductors with a rectangular cross-section (such as interconnects on PCBs), the current distribution is concentrated at the corners of the interconnects. Solving the diffusion equation for such conductors is done by methods such as the Finite Element Technique [26, 27]. In [28], these losses are incorporated in the Helmholtz equation, which is employed as a basis of analysis. The EM simulator OptEM VLSI, [29] is based on this technique.

The three methods widely used in solving Maxwell's equations for electromagnetic analysis are the Finite Difference Technique, the Finite Element Method, and the Method of Moments. These are described in books such as [26, 30].

The Finite Difference Method (FDM) is a method used to solve partial differential equations such as the Laplace Equation. A finite set of points in the region at which the potentials are to be determined is defined, and a set of linear algebraic equations 
describing the potentials at these points is solved to give the approximate values of the potentials at these points. It is apparent that for accurate results, a large number of points must be taken, and this would require a large CPU time.

The Finite Element Method (FEM) is similar in concept to the method of finite differences, except that it breaks the region down into a finite number of sub-regions. The electromagnetic field in each region is simple enough to be approximated by low-degree polynomials, which can be collectively analyzed using linear algebraic equations. Again, the level of accuracy of the results is dependent upon the number of elements into which the region is broken down.

The Method of Moments (MOM) relies not on breaking down the region and boundaries, but on decomposing the function itself into known functions. It is mathematically more complex, but gives greater computational efficiency. The linear partial differential or integral equations (e.g. the Laplace Equation) which are to be solved to obtain the RLCG parameters of a conductor are approximated to a linear combination of known functions, such as a set of pulse functions, which are less expensive to analyze. The details of the Method of Moments can be had from sources such as $[30,31]$.

\subsubsection{The Move towards Empirical Relationships}

In the past few years, much effort has gone into developing faster and more efficient tools for high-speed interconnect analysis, in order to further reduce run-times and computational intensity.

The techniques described in the previous sectious, no matter how fast and efficient, are still relatively too slow for total automation of design and optimization by the methods discussed in Section 2.3. The CPU run-time spent in obtaining design and 
analysis parameters should be small, so that a large number of calls to the function or technique would not result in impractically long run-times.

It has been noted in the literature that parallel to these approaches involving the determination of signal integrity parameters from first princıples (such as Maxwell's Equations, etc.) upon which all the above techniques are based, there have also been methods which use data to extrapolate or map input-output relationships in a blackbox approach. The data used to create these interconnect models, based on optimal curve-fit or other mapping techniques is either obtained by physical measurement or off-line simulation using traditional approaches. This technique has been studied as a general technique by many researchers $[7,32]$.

In [33], closed-form formulas for the lossy parameters ( $R$ and $L$ ) of a microstrip were developed by Djordjevic and Sarkar. In [34], substrate coupled switching noise in ICs is estimated based on previously measured data in order to speed up simulation. In [35], discontinuities in microstrips are modeled using lumped-equivalent models the component values of which are obtained using curve-fit techniques.

Several analytical techniques using electromagnetic analysis and transmission line models use polynomial curve-fit equations to describe the frequency dependence of the parameters such as propagation constant, characteristic impedance, etc. $[5,25]$. In [36], previously established empirical relationships are used to estimate interconnect capacitance in VLSI circuits. In [37], automatic polynomial curve-fitting is done for interconnect capacitances.

These techniques are capable of saving CPU resources, as well as much time and energy in developing and executing complicated algorithms from first principles. 


\subsection{CAD for Passive Microwave Components}

With the rapid advancement of MMIC (Monolithic Microwave Integrated Circuit) technology, there is a constant need for fast and efficient CAD tools in this area to assist in the design and analysis of active and passive components used in circuit design. Typical examples of passive components used in microwave and millimeter range ICs are microstrips and slotlines used as waveguides, thin film overlay capacitors, inter-digital capacitors, stubs for impedance matching, spiral inductors and spiral transformers. These components are used in a variety of applications in various microwave circuits $[38,39]$.

MMICs prominently use Gallium Arsenide as a substrate, however recently Silicon has gained importance in production technology, primarily due to its low cust. Due to their mass manufacturability, monolithic ICs have gained significance in several market-driven applications. A typical example of such an application is wireless communication for PCS (Personal Communication Systems), where final cost is a significant factor. Monolithic circuits also imply that once they are fabricated, virtually no tuning of parts or component values can be done. Thus the design and optimization stages are of utmost importance, and must be done intensely and thoroughly to ensure a high yield and proper performance after mass production. The importance of optimization and yield analysis for MMICs, with respect to component characteristics and process variations, is emphasized in several papers such as $[40,41]$.

Developing CAD models for circuits and components in the microwave and millimeter range involves accounting for many factors which come into play at these frequencies, and when components are bound together in such close quarters. The coupling effects between close conductors, skin effect, and ron-ideal dielectrics (espe- 
cially on a silicon substrate) are among these effects. Since the components normally have dimensions and inter-spacing in the micron range, these effects are quickly seen. Furthermore, circuits often have multi-layered components, which have more than one layer of metallization, or passivation layers and inter-metal dielectrics, which must be accounted for [39]. 3-D electromagnetic analysis of the structure is the only approximation-free method of modeling, however it is expensive both in terms of runtime and memory requirements. With the large number of complex structures, bends, vias, etc. encountered in a typical microwave circuit, the CPU requirements would be tremendous, and thus 3-D analysis cannot be used efficiently on-line in design and analysis [40].

In view of the unsuitability of electromagnetic simulators on-line, much research has gone into finding efficient techniques to characterize microwave components for use in automated design and analysis routines. While the use of tables and measured data for microwave component modeling dates back to the pre-war era, with Haefner's classical conductor loss measurements [42], and curve-fit techniques equally so, numerical simulation techniques have advanced in more recent years with the availability of fast computing resources.

As a specific case of a passive microwave component, techniques used to model the monolithic inductor are discussed in the next section.

\subsection{The Monolithic Inductor}

The spiral inductor is an efficient and economic method of implementing an on-chip lumped inductance. It has been recognized as being a vital element in many present day analog circuit applications. Since its implementation was first demonstrated 
on silicon technology by Nguyen and Meyer [43], the spiral inductor has found use in numerous applications such as low noise amplifiers, mixers, power amplifiers for RFICs. They have been identified in several review papers, such as $[44,45]$, as a necessary component in the rapidly emerging area of wireless communication.

The spiral inductor is a lumped and more space-efficient representation of the straight wire inductor. There are several geometrical representations including the square, rectangular, circular and octagonal inductor. Multi-layer spiral inductors have also been studied [46]. In order to get maximum inductance per-unit-area of metal, however, the square spiral inductor is most commonly used. It consists of several transmission lines wound together in a planar, 'wrap-around' fashion such that they form a square spiral.

The monolithic inductor must be optimized due to several factors. They are often the largest component on a chip and hence are expensive in terms of die real estate. Also, the area of a spiral inductor influences the resistance and parasitic capacitances, which alter the quality factor $(Q)$. Many applications require extensive optimization routines in order to obtain maximal performance characteristics. Delay lines such as those described in [47, pp.41-44] are optimized to use a minimum number of components (spiral inductors and overlay capacitances) for a given delay.

\subsection{Modeling the Monolithic Inductor}

The characterization of spiral inductor structures is a very challenging task. Monolithic inductors behave as transmission lines, and at high frequencies these structures perform poorly on the semi-conducting substrates used in manufacture. The accurate characterization of these structures at microwave frequencies requires analysis of the 
fringing fields, parasitics, ground-plane effects and, most importantly for silicon IC deaign, an analysis of the effect of the conductive substrate on the performance of the component.

\subsubsection{Electromagnetic Simulation}

Full-wave electromagnetic field-based modeling is the most accurate method of characterizing the distributed parameter nature of the microstrip inductor over a wide frequency range. Several commercial software programs are available to perform fullwave EM analysis, such as EM-Sonnet or Momentum from Hewlett-Packard. However full-wave analysis is extremely expensive CPU-wise, as it is based on numerical techniques which solve first-principle equations such as Maxwell's Equations [39]. Furthermore, since most practical circuits ccintain a number of inductor, the use of the model would be repetitive, and hence direct use of EM-based methods on-line is impractical [40]. Also, the efficient use of an EM solver which employs the above techniques demands a high degree of sophistication and expertise from the circuit designer.

\subsubsection{Physically-based Lumped Element Techniques}

Another technique is to to model the behaviour of the inductor from the circuit layout and substrate parameters using physically-based lumped-element models. The values of the lumped elements of the model are obtained by using closed-form equations or by static EM simulation. The methods described in [47, pp.11-28], [48] and [49] are based on such techniques. They provide speed and allow for integration into existing circuit solvers. In [50], which is the method employed to generate data for this research, the spiral inductor is decomposed into individual microstrip lines, 
of which is modeled by a lumped-element $\pi$-equivalent circuit. The parameters of the lumped-element model for each microstrip line are computed from the layout geometry, and the substrate and metallization properties. A combination of numerical techniques (for the quasi-static EM analysis), and closed-form relationships (to model the frequency-dependent parameters), is employed to calculate the individual elements of the lumped circuit. The circuit model for the complete inductor can then be directly used in a time-domain (e.g Spice) or frequency-domain (e.g. Touchstone) simulation along with other active and passive RF elements. This method is a balance between using EM simulation and lumped-element techniques, and as such is substantially faster than using 3-D EM simulation. However, as subsequent timing measurements will show, it is still too slow to be used in extensive optimization routines or parameter-sweep techniques.

\subsubsection{Using Measured Data}

The challenge of spiral inductor characterization is often met by using extensive measured data as a means of characterization. In [51], over a hundred spiral inductors were fabricated and general characterization done on the basis of the S-parameters measured. The characterization of spiral structures reported in [46] and [52] are also based on the curve-fitting of data. Pettenpaul's curve-fits described in [53] have been applied in several papers in the design and analysis of microwave components.

The non-availability of fast techniques for use in on-line circuit design and optimization has led to much interest in table look-1.p techniques, such as those discussed in [39]. An examr : $:$ of a table look-up implemented for inductor characterization is [54]. It has also been implemented in widely-used circuit simulators such as SPICE that have been enhanced for microwave design use, and other dedicated RF circuit 
simulation tools such as [55].

\subsection{Fast On-line Modeling in a CAD Environment}

From the above discussion of CAD techniques available for analysis of interconnects and microwave structures, it is apparent that accurate simulation involves solving EM field equations, transmission line equations, or using extensive lumped element models. The CPU intensity of these operations limits their practicality in iterative CAD methods, such as optimization, parameter sweeps, performance analysis, etc., as they would take too long to converge. Thus there is a need for fast models capable of driving such optimizers and design tools without using up too much $\mathrm{CPU}$ resource. This is shown in Fig. 2.1. There are several "front-end" simulation techniques available, the major çes are discussed here.

\subsubsection{Curve-fit and Closed-Form Models}

Curve-fitting is a general technique used to produce black-box models of input/output data, independent of the actual problem at hand. Methods adopted in curve-fitting include polynomials, piecewise techniques or splines $[7,32]$. These modeling techniques suffer from the disadvantage of being capable of handling only mild non-linearity, and a limited number of input variables.

The use of closed-form equations such as in $[33,49]$ is another technique related to curve-fitting. The main difference is that closed-form formulas are are not problem independent. Much research and effort must go into the development of each set of closed-form solutions, and extending the range of an input variable or slightly altering conditions might require an entirely new set of equations. 


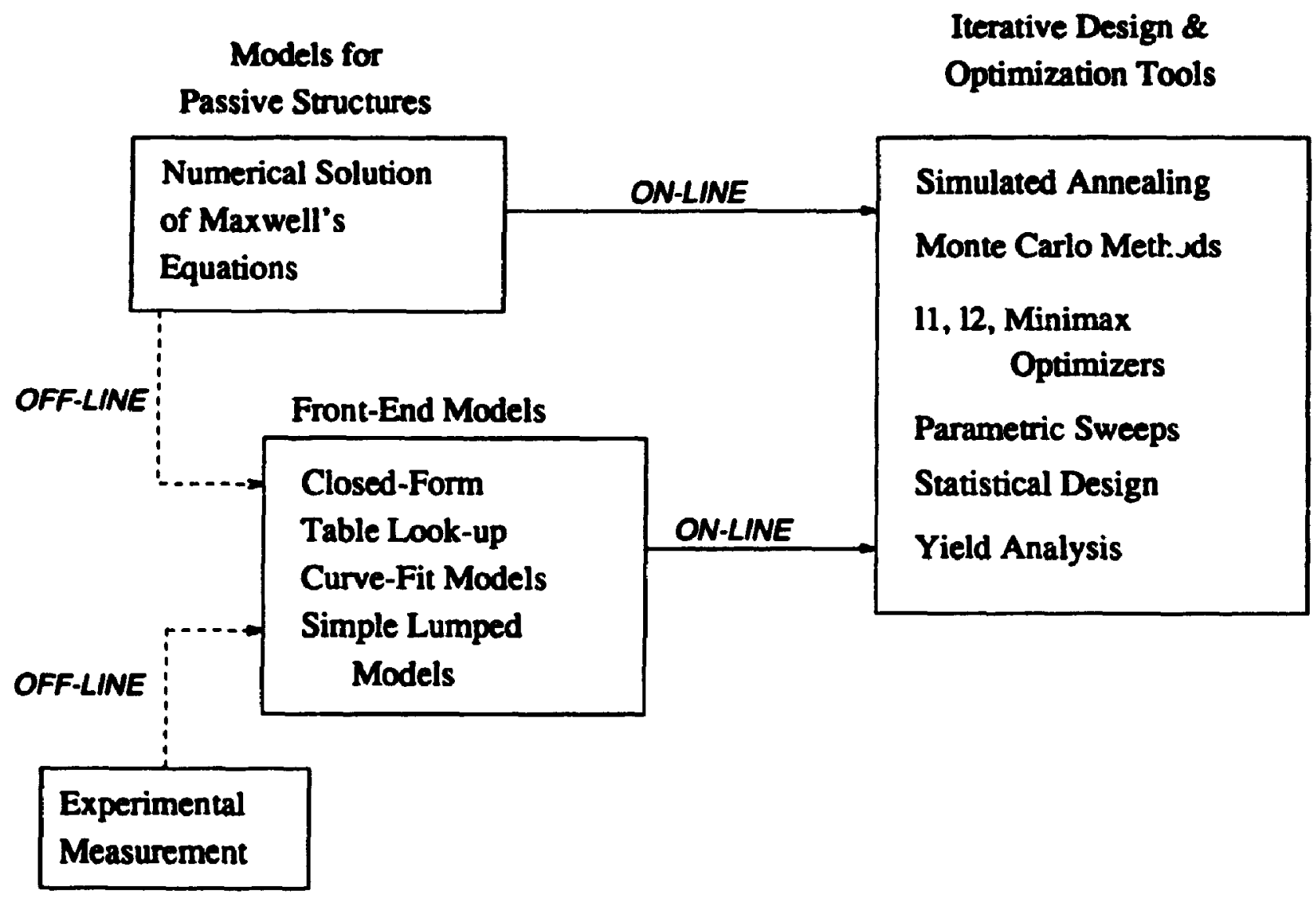

Figure 2.1: A typical CAD/optimization environment 


\subsubsection{Table Look-up Techniques}

The method of table look-up is a fast approach to on-line design, as the CPU time required is the trivial time taken for a search or a query on the table. It has been researched and implemented for interconnect analysis as well as passive microwave component modeling $[39,53]$. It is undoubtedly fast, but suffers from the following drawbacks:

- the size of a table grows exponentially with the addition of each new variable

- every entry in the table requires a data point, obtained either by measurement or computation

- raw data tables are cumbersome to maintain and upgrade, and hence are not very robust

- their accuracy is a function of the number of samples in the input space, and interpolation algorithms are not always accurate in high dimension

- the derivatives of the outputs with respect to the inputs do not explicitly exist, hence sensitivity analysis is not possible.

\subsubsection{Simple Lumped-Element Representation}

Another method currently used to expedite on-line simulation is the use of simple lumped-element circuit representations of the complex structure to be modeled. The passive structure under study is mapped on to a simple circuit containing three or four lumped elements (resistors, capacitors and inductors). The choice of elements and their values depends on the structure, the values assigned based on the data obtained through off-line simulation or measurement. 
A typical example of the use of lumped-element representations is [35], where discontinuities in microstrips are modeled.

Lumped-circuit models are easily incorporated into electrical simulators (such as Spice), but suffer from the disadvantage that they are normally highly restrictive on the range of frequency over which they are valid. Also, the choice of the correct lumped circuit configuration, and the values of the lumped elements is a difficult task.

The underlying philosophy of all the above methods is to base on-line simulation techniques on data which has already been acquired, either as such or in an interpolated form, rather than using an extensive first-principle based algorithm on-line every time an analysis must be performed. The extensive calculations or measurements and pre-characterization are performed off-line, and the results obtained used on-line.

In the following chapters, the application of neural networks to on-line simulat:on, as an alternative technique for fast front-end simulation is investigated.

\subsection{Summary}

This chapter has provided a conceptual background to the CAD and analysis of highspeed VLSI interconnects and passive components in RF circuit design. Traditional methods and research efforts in these areas have been surveyed. Current techniques adopted to expedite on-line simulation, their limitations, and the need for a general method for this purpose, have been described. 


\section{Chapter 3}

\section{Neural Networks: The Concept}

\subsection{Introduction}

In this chapter a conceptual overview of neural networks is presented. A brief account of the history of neural network research is provided. The structure and working principles of the main types of feed-forward and feedback networks are described. There are several specific types of neural networks, but it is seen that the underlying principles and building blocks are common to them all. The overview presented in this chapter provides insight into the general concept of problem solving using neural networks. In the last section, several of the applications neural networks have found in science and engineering are listed, and the suitability of neural networks to CAD problems explored.

\subsection{Early Research}

The neural network was originally an attempt to model the learning process in human beings, i.e. to produce a model capable of learning and thinking in a manner identical to the human brain. It was known that brain activity was performed by points in the 
brain called neurons, which existed in highly interconnected networks. Most of the early research in neural activity was carried out by biophysicists and psychologists.

One of the first descriptions of neural activity in the brain is attributed to William James, a scientist in the area of the physiology and psychology of the brain. In 1890, he stated that "the amount of activity at any given point in the brain cortex is the sum of the tendencies of all other points to discharge into it," and further that this tendency was proportional to the past activity of the points in question, the intensity of the excitement, and the presence or absence of inhibitory excitations.

In 1943, McCulloch and Pitts used this description to mathematically model the neuron [56]. The model they formulated was to become the building block of most neural networks used to date. The structure of the McCulloch-Pitts model is shown in Fig. 3.1, and is described in detail in the following section.

Neural network research continued to grow in the post-war years through to the sixties, with developments such as Adaline in 1963 and Madaline [57, 58]. In 1969, however, research in neural networks came to a virtual halt, due largely to the infamous book of Minksy and Papert [59]. The book stated and proved that the single layer perceptron was incapable of solving any problem which was not linearly separable. A problem which is linearly inseparable is one in which the input space cannot be linearly divided into compartments using linear hyperplanes, such that each compartment map onto one state of the output. The XOR gate is a classical example of such a problem. Minsky and Papert continued to state that further research into multi-layered networks of perceptrons (neural networks as they are known today) would be "sterile", thereby effectively halting research in neural networks for about a decade.

Research efforts in neural networks were revived in the late seventies with the 
advancement of computing technology and the availability of more powerful computing capabilities. Since then, efficient algorithms have been developed for the design and implementation of neural networks, several architectures have been proposed, and neural networks have found applications in many different areas of the physical sciences and engineering.

\subsubsection{The McCulloch-Pitts Model}

The McCulloch-Pitts model of the neuron is shown in Fig. 3.1. Also termed the perceptron, it is the basic building block of most common neural network architectures.

The perceptron has input nodes $x_{i}, i=1,2, \ldots m$, which are linearly combined through a set of interconnection weights $w_{i}, i=1,2, \ldots m$. The resultant combination, $\gamma$, given as

$$
\gamma=\sum_{i=1}^{m} x_{i} w_{i}
$$

is passed through a transfer function, which may be linear or non-linear, to give the output of the neuron, $z$ :

$$
z=f(\gamma)
$$

Different kinds of transfer functions coinmonly used are shown in Fig. 3.2.

In relation to James' original statement, output of the neuron is dependent upon the number of points discharging into it $(m)$ and the intensity of their discharges, $\left(x_{i}\right)$. The presence or absence of inhibitory signals is determined by the sign of the interconnection weights $w_{i}$, and the correlation with past activity is represented by the values of the interconnection weights, which are normally adjusted either through 


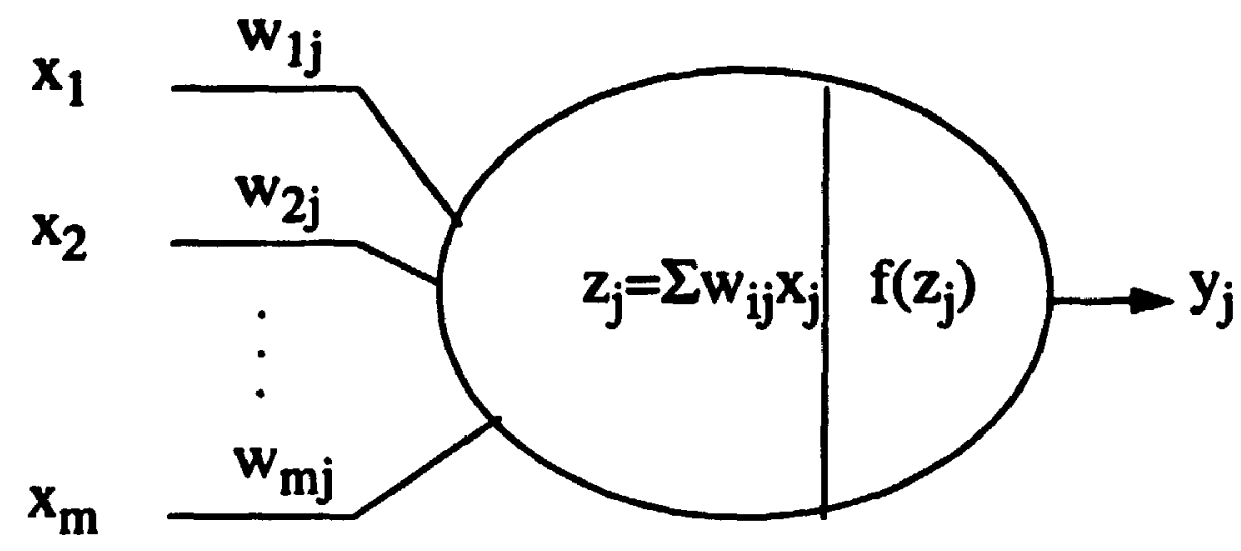

Figure 3.1: The McCulloch-Pitts model

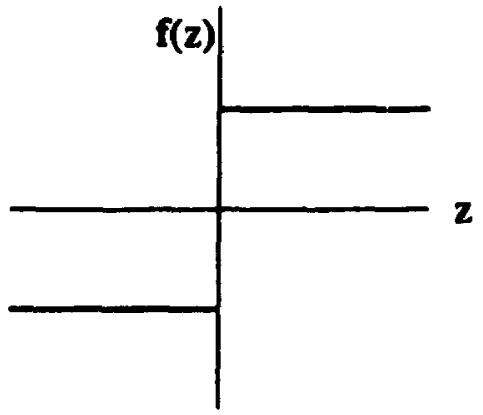

Hardlimiting

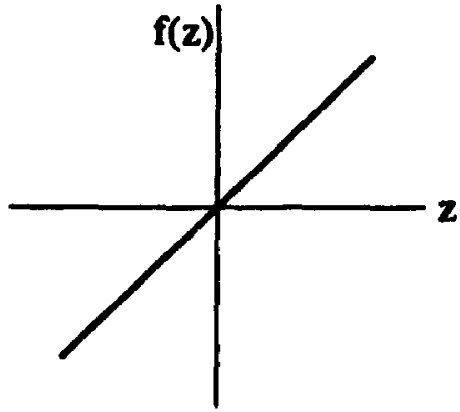

Linear

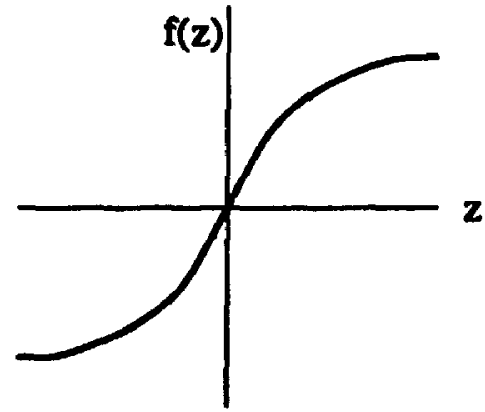

Sigmoidal

Figure 3.2: Different kinds of transfer functions 
training or otherwise, based upon a knowledge of how the neuron is to reapond to different stimulations (inputs).

The function $f(z)$ can be chosen from a variety of functions, either as a linear operator, a hard-limiting threshold operator which only takes values 1 or 0 depending on $z$, or a non-linear function such as the sigmoid. The choice of function largely depends upon the application and the nature of the learning process. Typical examples of transfer functions are shown in Fig. 3.2.

The ability of a perceptron to respond correctly to its input lies in the values assigned to the interconnection weights, which are defined through the learning process. Learning algorithms can be classified into two broad categories:

Supervised learning is the type of learning when the actual responses to a set of stimulations are known beforehand. In other words, given a set of input/output data, learning implies determining the correct mapping between the two data sets.

Unsupervised learning occurs when the output is not known beforehand. The weights are adjusted solely on the basis of the set of input data available.

Supervised learning is used in all function-mapping applications, including those in this thesis, and trains a neural network to recognize the solution to a problem on the basis of past experience and previously encountered data through a process of interpolation and mapping of known information. Unsupervised learning is useful in problems involving the classification of data or patterns, or in the identification of clusters of data within a large space.

The next two sections attempt to classify the several neural network structures and learning rules currently available. 


\subsection{Feed-Forward Neural Networks}

In a feed-forward neural network, no feedback paths exist from the output to the input during operation. Computations are performed in a single pass (although through multiple layers) from the input to the output. Feed-forward neural networks are very popular and have several applications because of the simplicity of their training algorithms, and their inherent stability given the absence of feedbrack paths. Adaline and the backpropagation network are the two most important types of feed-forward neural networks.

\subsubsection{Adaline and The Delta Rule}

The ADALINE, which stands for ADaptive LINear Element, was developed by Widrow and Hoff [57]. It consists of a single McCulloch-Pitts neuron, with a linear transfer function, i.e.,

$$
z=f(\gamma)=\gamma
$$

It was employed to associate input patterns with outputs. The learning rule used to train ADALINE was the supervised Delta Rule, or the Normalized Least Mean Square Rule. Given an input vector $X$ whose desired output is $\hat{y}$, if the neuron calculates the output as $y$ using Equations $3.1-3.3$, then the weights $w$, which are initially assigned random values, are updated by

$$
\mathbf{w}_{\text {new }}-\mathbf{w}_{\text {old }}=\frac{\eta E X}{\|X\|^{2}}
$$

where $E=\hat{y}-y$ is the error, and $\eta$ is the learning rate parameter, which is the step size in the learning process. 


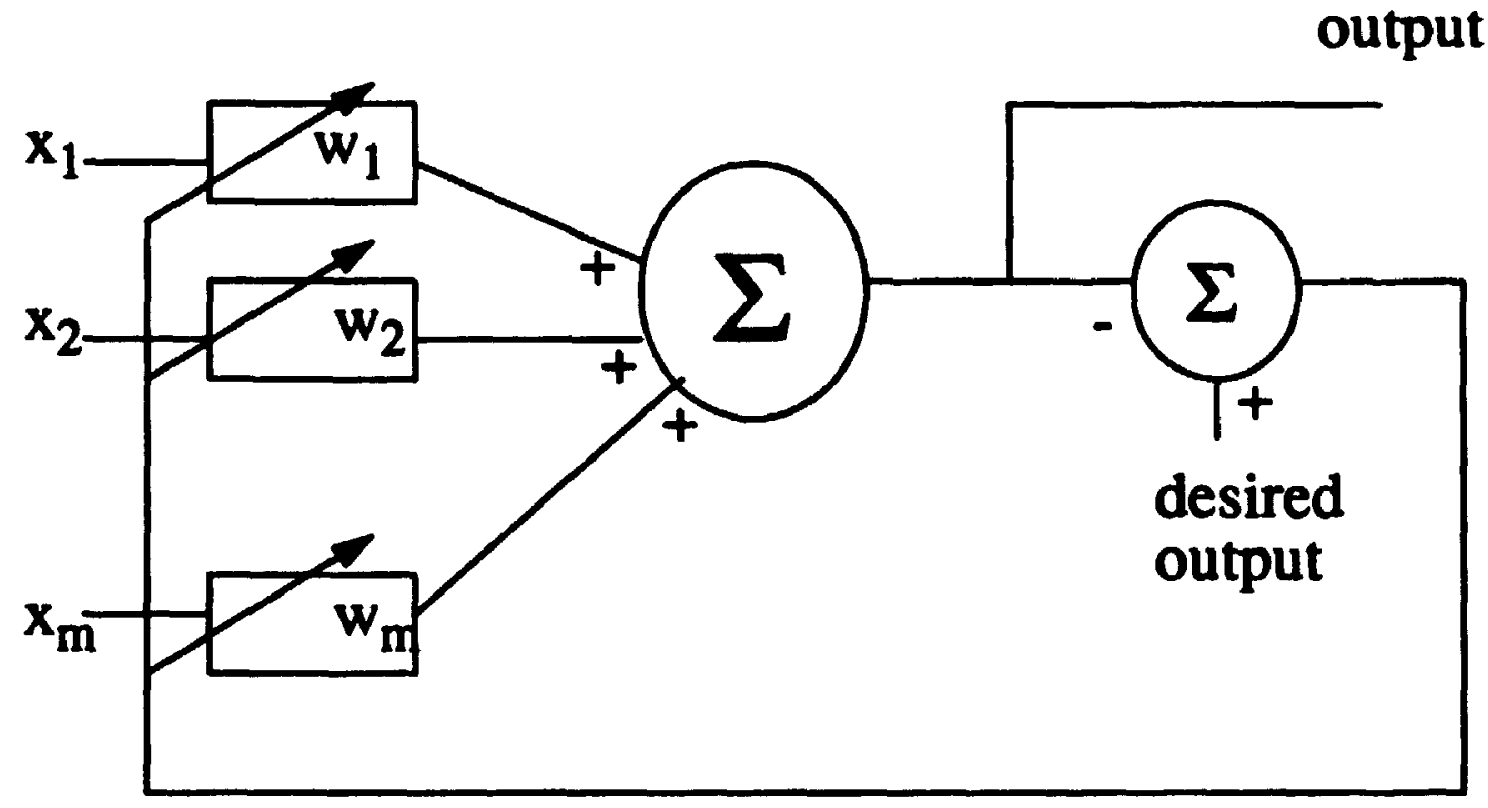

Figure 3.3: Adaline 
Adaline is shown in Fig. 3.3. The figure also shows the connections during training, and how the output passed forward is used in updating the weights. Applications of Adaline were relatively simple linearly separable problems. Adaline failed to solve linearly inseparable problems, and hence could not be applied to more complex problems.

\subsubsection{Madaline}

The successor of Adaline was MADALINE, or Many ADALINEs. Also developed by Widrow and Hoff [58], it basically consisted of several perceptrons connected in a single layer, to fixed logic gates (such as the AND or OR gate) at the output. This enabled linearly inseparable problems, in particular the XOR problem, to be solved.

\subsubsection{The Backpropagation Network}

The backpropagation network is essentially an extension of MADALINE, with two fundamental enhancements:

- several layers of perceptrons are present, the output of each layer acting as the input of the next

- the transfer function is the non-linear sigmoid.

Normally, they are three-layered, having an input layer, a hidden layer, and an output layer. If desired, there can be more than one hidden layer, however for most applications a one is sufficient. The learning algorithm is supervised, and is called the backpropagation algorithm. The backpropagation network is widely used in function mapping, and is the neural network chosen for the applications in this thesis. 
A detailed iscription of the structure, training and testing of the backpropagation network are given in the next chapter.

\subsection{Feedback Networks}

Feedback networks encompass all neural network structures in which there is feedback from the output to the input layers, or which allow lateral interaction between the neurons during normal operation. Lateral interaction is a useful concept as it allows for the parallel usage of information from other output nodes vhile determining an output. The main neural networks which fall under this category are described in this section.

\subsubsection{Hebb's Law}

Hebb's law is an important theory of learning in neural network theory and research. It describes the interaction of neural activity between neurons which are simultaneously active or responding to stimuli, even if they are responding to different stimuli. It is the basis of competitive learning and, though it is not linked with a particular neural network architecture, applies in general to most neural networks of the feedback category.

Hebb's law essentially states that neurons and neural activity are sensitive to the activity and outputs of neighbouring neurons as well as their own inputs, even if the neurons are not connected to the same input. The outputs of neurons have the capability of conditioning the responses of neurons in its neighbourhood, either reinforcing the stimulus or inhibiting it. The doctrine was mathematically quantified by Grossberg, and learning on the basis of Hebb's Law is referred to as Grossberg learning [60]. 
Given a network of fully interconnected neurons, i.e., one in which each neuron has external inputs as well as inputs from other neurons in the network, the dynamics of the $j$ th neuron are described by the following equation:

$$
\frac{\partial y_{j}(t)}{\partial t}=-A y_{j}(t)+I_{j}(t)+\sum_{i=1}^{n} w_{i j} y_{i}(t)
$$

where $y_{j}$ is the output of the $j$ th neuron, $I_{j}$ is the external input to the neuron, $w_{i}$, the weight connecting the output of the $i$ th neuron to the input of the $j$ th neuron, and $A$ is a positive constant controlling the decay of the output in the absence of other inputs. This equation implies that if the weight matrix is strong enough, the outputs of peer neurons could influence the output of a given neuron in a manner identical to the exierna! inputs.

This principle is the basis for several networks which operate with lateral interaction and feedback.

\subsubsection{Kohonen Self-Organizing Maps}

The Kohonen self-organizing network is a feedback network which is based on unsupervised learning. Its primary function is classification, or organizing data into different classes. During learning, the space that encompasses the data set presented to the network is broken down into different clusters. Groups of points existing closely together are classified as belonging to the same class, and thus the centres of the clusters are identified as being a class of data. During operation, when a new data point is seen, based on its distance from the different cluster centres, the network can classify it into one of the pre-identified classes.

The learning process of the Kohonen network is also referred to as being competitive, as the output is generated by a competition of sorts amongst the output 
neurons. By a system of strong positive and negative feedback, the neurons with larger outputs get larger and cause, through lateral interaction, a reduction in the values of other neurons in the output layer.

The structure of a Kohonen network is as shown in Fig. 3.4. All the neurons are fully interconnected, i.e. the output of each neuron is connected to the inputs of all neurons including itself, through a set of variable weights, $w_{i,}, i=1,2, \ldots m$ and $j=1,2, \ldots n$, where $m$ is the size of the input vector $X$, and there are $n$ neurons at the output. That the network is assigned $n$ outputs implies that the network is intended to organize the input data into $n$ classes (which may not be exclusive or even exist). The learning process is described by the equation

$$
w_{i, \text { new }}-w_{i, \text { old }}=\eta(t)\left(x-w_{i}\right) U\left(y_{i}\right)
$$

where $w_{i}$ is the weight vector of the $i$ th unit, and $x$ is the input vector. $\eta(t)$ is the time-variant learning rate parameter. $y_{i}$, the output of the $i$ th output neuron, is conditioned by the function $U(y)$, such that $U\left(y_{i}\right)=0$ if $y_{i}=0$, otherwise $U\left(y_{i}\right)=1$. This restricts participation in the learning process to neurons whose output is nonzero. By repeated presentation of the data set to the network, it will begin to recognize clusters, such that each cluster will be identified by a single neuron taking the value 1 , and the others zero. The centres of these clusters are stored in the weight vector.

After training, if a vector, $x$ is presented to it during operation, the winning output $c$ is determined competitiv'ly based on the similarity between the input vector and the weight vectors, represented as

$$
\left\|x-w_{c}\right\|=\min _{i}\left\|x-w_{i}\right\|
$$


The above is a simplified representation of the Kohonen network. More sophisticated algorithms have been developed to improve upon this basic structure. Applications of self-organized maps include image and spatial pattern recognition, pattern classification, etc. [61].

\subsubsection{Bidirectional Associative Memory (BAM)}

The Bidirectional Associacive Memory (BAM) is an important member of the class of neural networks dealing with associative memory. The main principle behind associative memory is the association of data with other data instead of with addresses. If a set of vectors $\mathbf{X}$ is associated with corresponding vectors $\mathbf{Y}$ through an associative memory, a given member of $\mathbf{X}$ can be used to retrieve its corresponding $\mathbf{Y}$ element, even in the presence of noise.

The architecture of a BAM is shown in Fig. 3.5. It has two layers of fully interconnected neurons. The weight matrix $\mathbf{w}$ is obtained as:

$$
w=y_{1} x_{1}^{t}+y_{2} x_{2}^{t}+\ldots+y_{q} x_{q}^{t}
$$

where $q$ is the total number of vectors to be memorized. The structure with the above weights can be used to recall the vectors stored in the BAM, even in the presence of noise, or if one of the vectors is only partially known.

Information is recalled by feeding the noisy vector available to the input (the $x$ layer) and, after successive propagation back and forth through the weighted interconnections between the two layers (the $x$ and $y$ layers), the values at the output (the $y$ layer) stakilize to the correct value. During this stabilization, the $x$ vector at the input also stabilizes to its noise-free value.

This method can be adopted in the reverse direction as well, i.e., if a given $y$ 


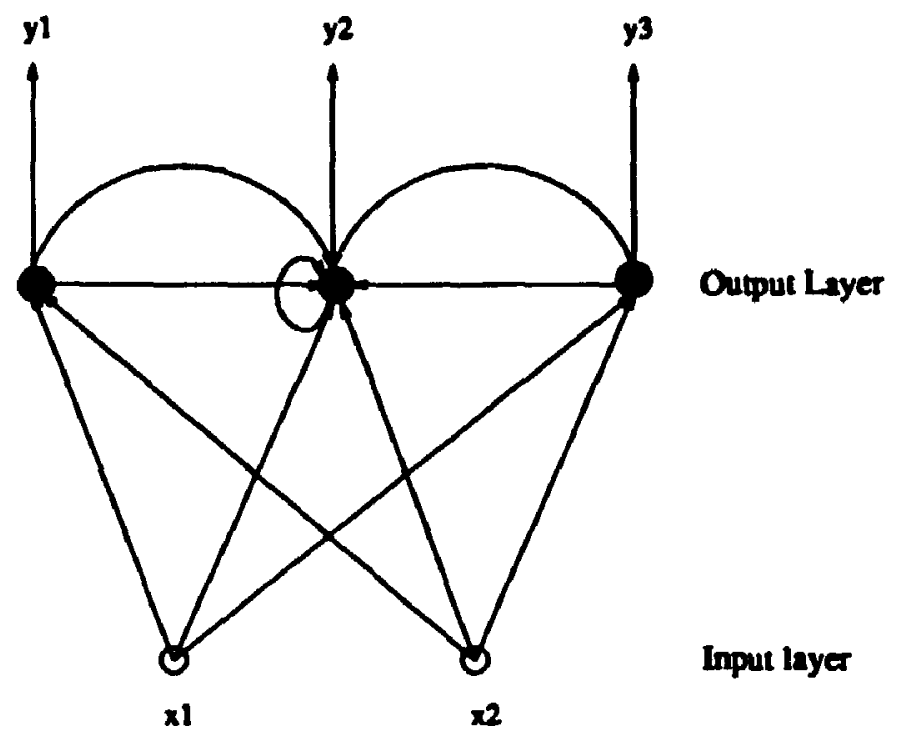

Figure 3.4: The Kohonen Self-Organizing Network. Feed-forward and feedback connections are shown, the feedback only indicated for the centre neuron of the output. layer

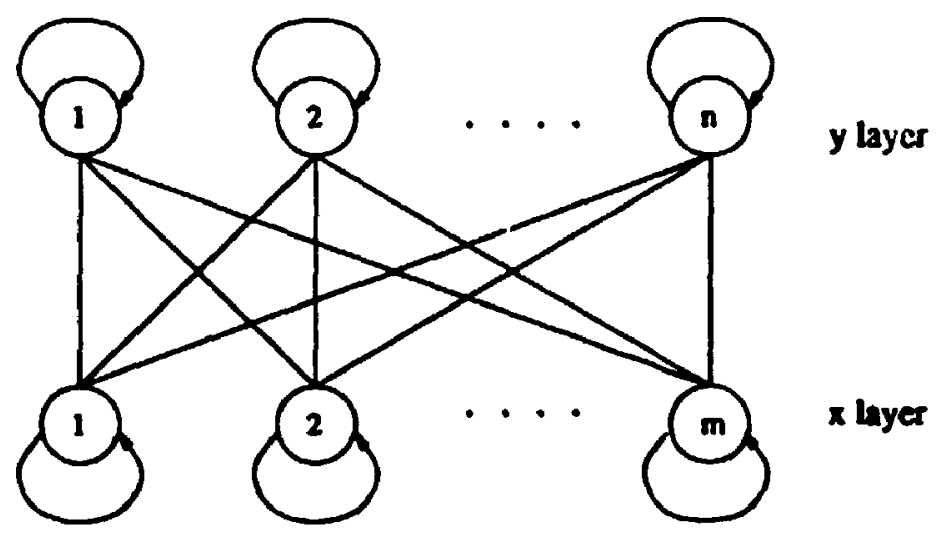

Figure 3.5: The Bidirectional Associative Memory (BAM) structure 
vector is known, it can be used to determine its corresponding $x$-vector. This is the reason for the name "bidirectional".

\subsubsection{The Spatio-Temporal Network (STN)}

The spatio-temporal network is an attempt to identify sequences of images or patterns using neural networks. Given a certain set of sequential images or frames, they can be identified or classified in a manner similar to the classification of a single image, except that they represent a multiple set of images being treated as a single entity.

Given a sequence of images stored in frames, its mere size prevents the usage of a single input layer for the entire input vector. Rather, alternative approaches such as the finite state method are applied to reduce the size of the network. Applications include speech recognition and image processing [62].

\subsection{Applications of Neural Networks}

Neural networks have been applied to a wide spectrum of problems in the physical sciences and engineering. Neural network applications have been treated extensively in the literature, both in books such as $[61,62]$, as well as in numerous publications and conference proceedings. A few of the applications of neural networks are described in this section, in an attempt to depict the versatility of the approach and the ease with which it can be adapted to any discipline.

\section{Control Systems and Robotics}

Neural networks have been applied in designing control systems for motors, etc. [63], and for designing robot arms and controllers $[61,64,65]$. Both feedback and feedforward structures have been used in these applications. 


\section{Pattern Recognition, Signal/Image Processing}

Pattern recognition is an important area in which neural networks have found applications. The power of neurons to recognize patterns in the presence of noise has enabled several applications in this area. This includes optical character recognition and handwritten character recognition [61].

Signal and image processing applications in medicine include image processing or feature extraction from visual devices such as computer tomography, or magnetic resonance imaging (MRI) $[66,67]$ and the analysis of biopotential signals such as the electro-cardiogram (ECG) and the electro-encephalogram (EEG) [68].

\section{Speech Recognition}

Speech recognition is another area in which neural networks have been extensively applied. Architectures employed for speech recognition include feedback, feed-forward, and combinations of the two principles to allow supervised learning in self-organizing structures, as in learning vector quantization (LVQ) [61, 69]. Several da a compression techniques are also based on neural networks.

\section{Industrial Process Modeling, Fault Detection}

Process modeling using neural networks has also gained popularity in recent years. The capability of the backpropagation algorithm to identify cause-effect relationships between sets of data, even based on relatively small amounts of training data available, has given rise to neural network applications such as modeling of systems performance estimation and prediction, plant monitoring and evaluation [71]. Huang and Zhang identify numerous areas in which neural networks can be applied in the area of manufacturing [70]. In [72], Li et al. make a comparison between statistics- 
based models and neural network models, and find neural networks to be of greater accuracy and better suited for PCB yield analysis studies. IC fabrication processes such as ion-etching have also been successfully modeled with feed-forward neural network structures $[73,74]$. Problems such as cereal grain classification in agricultural process monitoring have also been explored by neural network researchers [75].

\section{Non-Engineering Applications}

Neural networks have also found numerous applications in the social sciences. Much media attention has been given to applications such as modeling financial and business phenomena such as stock trading, etc. In [76], Wilson employs self-organizing maps to model common stock trading. Stock market prediction based on mulli-layer perceptrons is done in [77]. Prediction based on past statistics, such as in disease control or mortality is also a well suited application of neural network methods, e.g. [78].

\section{Modeling of Electrical Systems}

Neural networks have been used in characterizing electrical and electronic systems such as devices and circuits.

The voltage-current characteristics of induction motor controls and the coupling characteristics of lasers have been modeled using neural networks $[63,79]$. The approach has also been used in modeling thick-film resistors [80], and silicon dioxide films [81]. These problems were all characterized by the lack of efficient analytical formulas and multi-dimensional relationships inadequately represented by empirical formulas.

In [82], neural networks are applied to identifying errors and failure patterns in 
SRAMs. Kant and Drezen, in [83], have applied Hopfield Networks to static electromagnetic field computation. In [84] and [85], Vaj et al. have applied feedback neural networks to microwave problems such as impedance matching, and to Smith Chart analysis. The optimization of electromagnetic devices using neural networks in conjunction with gradient-based methods has been studied in [86].

Initial efforts in using feed-forward neural networks as CAD tools have been successful. In [87], Zhang and Nakhla demonstrate the use neural networks in yield optimization in passive filters and coupled transmission lines. Neural network-based models for interconnect analysis and optimization have reported accurate results and substantial on-line speedup over existing techniques $[88,89]$.

Much work has been done by several researchers concurrent to this thesis in this area. This includes research such as microwave circuit and device modeling for statistical design by Zaabab et al. [90], modeling operational transconductance amplifiers by Kothapalli [91], and Si-based MOSFET modeling by Ojala et al. [92]. This gives and indication of the suitability of the neural network approach to CAD tool development.

\subsection{In Context: Neural Networks in High Fre- quency Applications}

This section discusses the suitability of neural networks to the application of modeling and simulation at high frequencies.

As stated in Chapter 2, the main difficulty with electromagnetic methods available for simulation and modeling at high frequencies is the large computational time involved. This high CPU intensity is caused by the complexity of the algorithms used 
in traditional methods, and disallows their direct integration into on-line CAD and optimization routines.

Neural networks differ in their approach to modeling and simulation problems. Their approach is a black-box technique which employs past experience and instances as the basis for determining the output for an instance presented to it. After having seen several input/output pairs, it is possible to interpolate the relationship seen in these pairs to determine the output for any input. The following aspects separate this approach from traditional computing approaches:

- neural networks do not sequentially execute elaborate sets of instructions, and hence do not require memory for storing complex instruction sets, several variables and data

- neural networks respond to inputs in parallel

- the approach is a transformation, or multiple transformations, as opposed to being an algorithm or routine based on executing a step-by-step procedure.

The neural network thus operates on the basis of transformations, determining the best transformation or mapping of a given set of data onto a predefined output. Once this transformation is learned, any input set could be subjected to this transformation to give a corresponding output. The transformation, which constitutes one forward pass of data through a neural network, is computationally very simple, and thus can be used to expedite on-line CAD and optimization routines.

As the computational time is very small, it can be used as a fast front-end simulation technique developed off-line, as an efficient alternative to existing front-end techniques. This is shown in Fig. 3.6, which is a modification of Fig. 2.1, and is the subject of this thesis. 


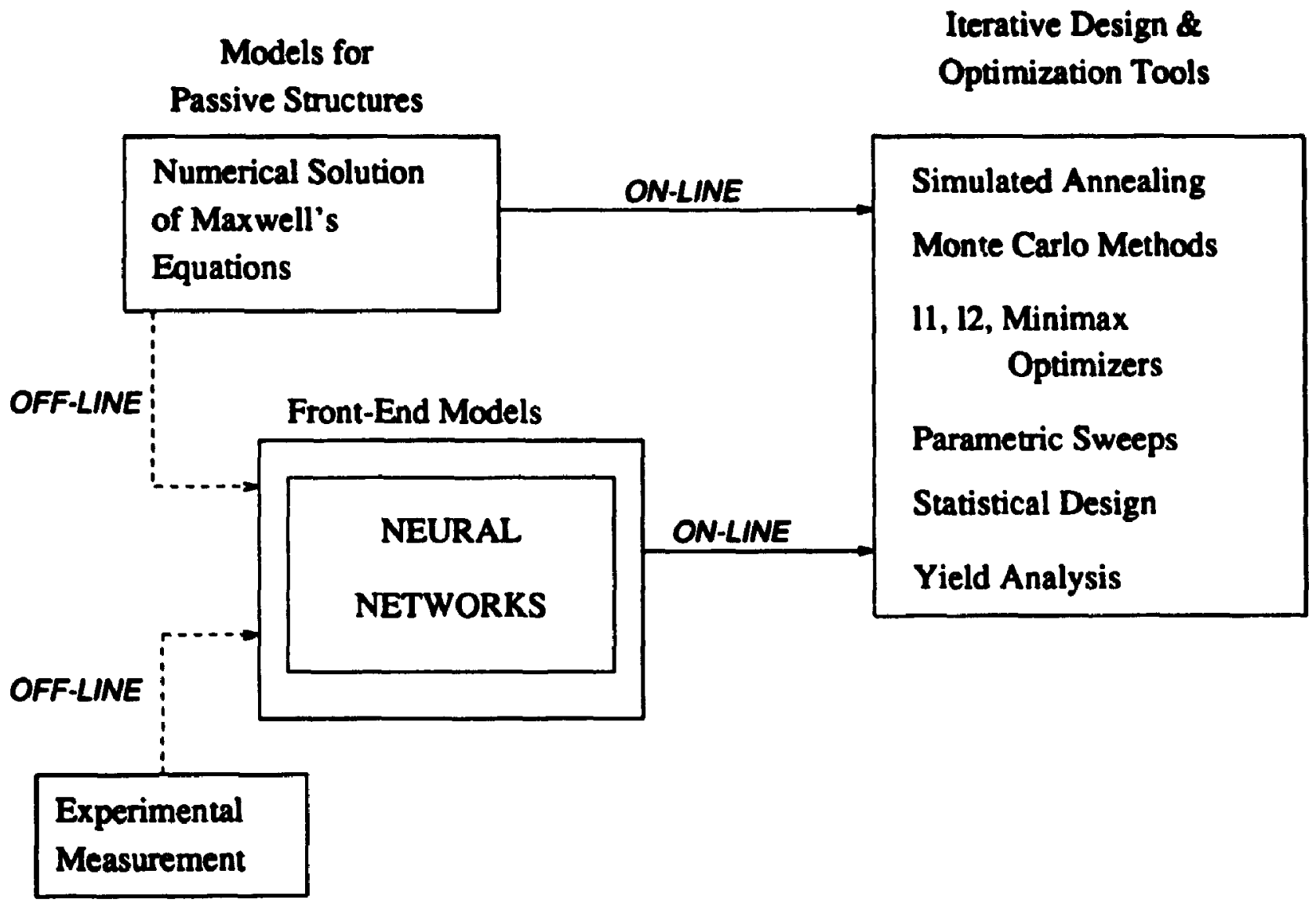

Figure 3.6: A neural network-based CAD environment 


\subsection{Summary}

This chapter has reviewed the concepts of learning and applications of neural networks. The capacity of neural networks to learn relationships and classify data has made the neural network approach very popular, and thus their applications span a wide range of problems. Reports of these recent applications prompt their use in CAD and optimization techniques as a method of reducing the run-times and CPU intensity of traditional approaches. 


\section{Chapter 4}

\section{The Neural Network Model}

\subsection{Introduction}

In this chapter, the structure of the neural network model, and the general approach to formulating a high-speed interconnect or passive microwave structure problem into a neural network model is described. Methods for generating the data required for construction of the neural network model, and the backpropagation training algorithm are explained.

\subsection{The Backpropagation Neural Network}

The type of neural network best suited for modeling and function mapping is the backpropagation network. As seen in the previous chapter, the backpropagation network is a feed-forward neural network based on the supervised learning principle. It has a training algorithm which is efficient, simple and easily implemented. The neural network as such is inherently stable due to the absence of feedback.

The three-layered backpropagation network can be mathematically described as a tool which relates an input space to an output space by a non-linear relationship ir 
has learned over the training process. The input space, $\mathbf{X}$ of dimension $m$, is mapped to its corresponding $n$-dimensional output space by the function

$$
\mathbf{Y}=\mathcal{F}(\mathbf{X})
$$

The $n$ elements of the output vector are represented as a layer of $n$ neurons, which are connected to the $m$ nodes of the input vector through the hidden layer. This hidden layer has a fixed number of neurons, $p$, which can vary from problem to problem as will be discussed in the next section. The output of any given neuron is the weighted linear combination of the outputs of all the neurons in the previous layer reflected off the sigmoidal transfer function. The neural network is pictorially represented in Fig. 4.1.

The mapping relationship is mathematically described as the relationship between the set of input vectors $X$, whose $k$ th sample is

$$
x_{k}=\left(\begin{array}{llll}
x_{k 1} & x_{k 2} & \ldots & x_{k m}
\end{array}\right)
$$

to the corresponding output vector,

$$
y_{k}=\left(\begin{array}{llll}
y_{k 1} & y_{k 2} & \ldots & y_{k n}
\end{array}\right)
$$

through a system of weighting factors and biases. The weights as $w_{i h}, b_{h}$, for $i=$ $1,2, \ldots, m$ and $h=1,2, \ldots p$ connect the input layer to the neurons of the hidden layer, and $v_{h j}, c_{j}$, for $h=1,2, \ldots, p$ and $j=1,2, \ldots n$, connect the hidden layer to the outputs, such that the $n$ outputs are

$$
y_{j}=f\left(\zeta_{j}\right)=\frac{1}{1+e^{-\zeta^{j}}}
$$




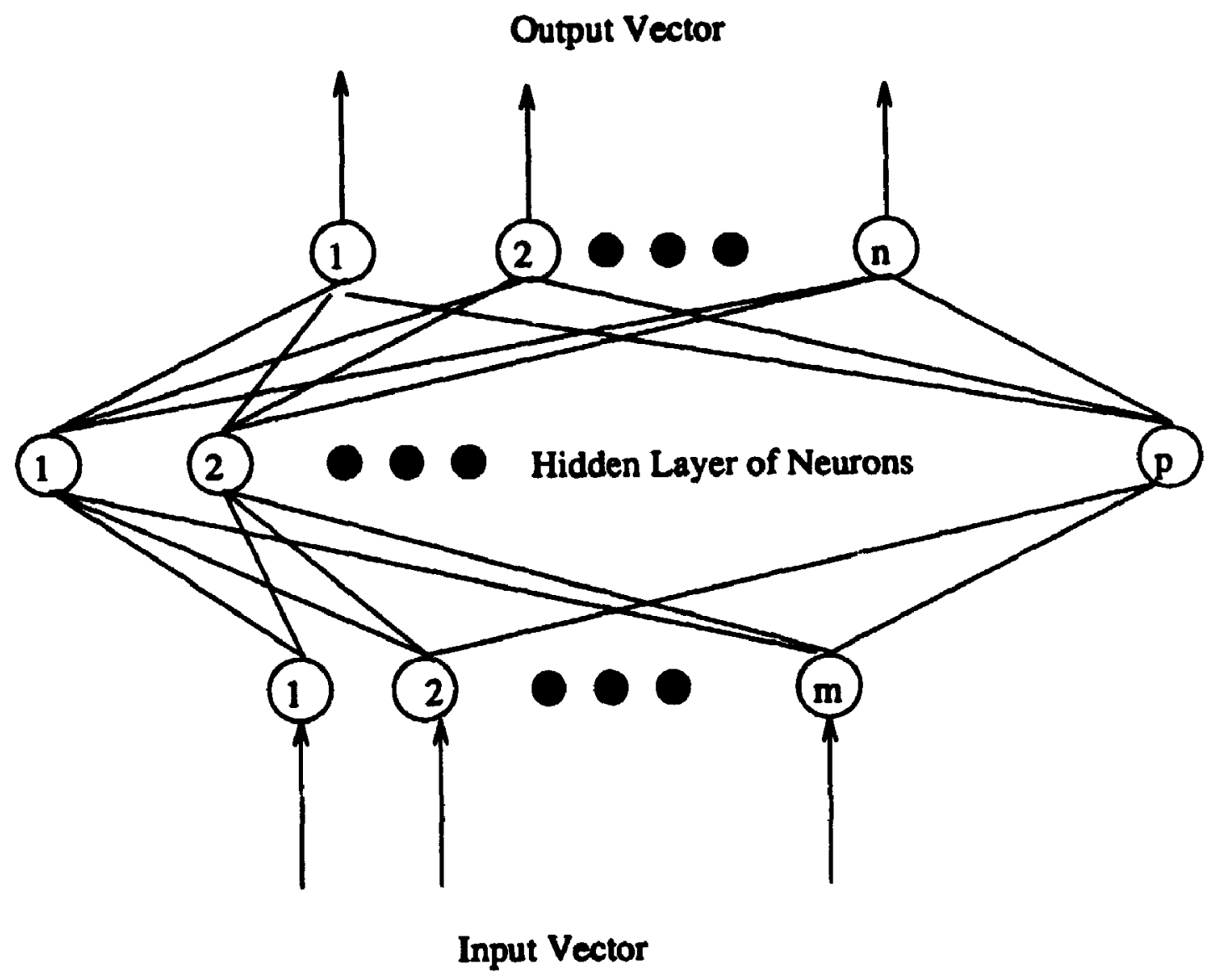

Figure 4.1: Structure of the Neural Network Model 
where

$$
\zeta_{j}=\sum_{h=1}^{p} z_{h} v_{h j}+c_{j}
$$

Here, $f(\zeta)$ is the sigmoidal transfer function, and $z_{h}$ is the output of the $h$ th neuron in the hidden layer, calculated as

$$
\sim_{h}=f\left(\gamma_{h}\right)=\frac{1}{1+e^{-\gamma_{h}}}
$$

where

$$
\gamma_{h}=\sum_{i=1}^{m} x_{k i} w_{i h}+b_{h}
$$

It is seen that $\mathbf{X}$ is now related to $\mathbf{Y}$ by a set of sample data. If the set of samples $\mathbf{x}_{k}, k=1,2, \ldots Q$ is chosen such that it is representative of the entire input/output space, then the objective mapping function $\mathcal{F}$ is in effect learned by the neural network, based on the $Q$ points to which it has been exposed.

Since the input/output relationship expressed in Equations 4.4-4.7 has only used two basic arithmetic operations, i.e. the summing of products and exponentiativ. , the computational run-time required to calculate $\mathcal{F}(\mathbf{X})$ is trivially small, and thus the resultant model is ensured to have a low run-time.

\subsection{Construction of The Neural Network Model}

\subsubsection{Input/Output Parameters}

The general neural network model can be applied to high frequency analysis problems by defining the veciors $X$ and $Y$ appropriately, and configuring the network in such a way that Equation (4.1) can be mapped. 
Output modeled

(RLCG Matrices, Delay, S-Parameters, etc)

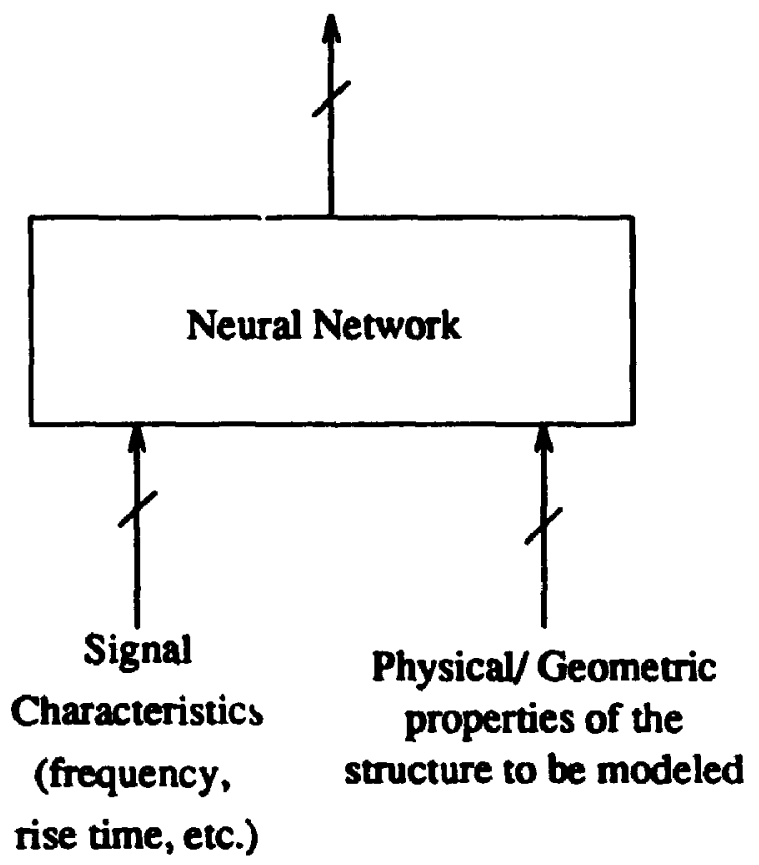

Figure 4.2: General structure of the model to be developed 


\section{The Input Layer: $X$}

The input layer of the neural network consists of $m$ nodes, representing the elements of the $\mathbf{X}$ vector. This contains information about the structure to be modeled, in explicitly parameterized form. Typical paraneters in the input set are the physical dimensions of the interconnect or spiral inductor, and their dielectric substrate characteristics, topology of the interconnect network, input signal characteristics such as voltage level, frequency of operation (or rise-time in the case of a digital circuit), termination impedances, etc., as shown in Fig. 4.2. Variables such as the layout topology of an interconnection network, which are implicit in netlist approaches, must be quantified explicitly.

Network topology can be quantified using graph theory, by representing the interconnection network as a directed tree rooted at the source, or any other pin of interest. The set of variables which represent the nodes at which a given interconnect has commenced (the source vertices of the edges of the rooted spanning tree describing the network) uniquely represents the network configuration. This is further described in Section 6.2.2.

\section{The Output Layer: $Y$}

The output of the model are the signal integrity parameters to be modeled, such as the signal propagation delay, crosstalk levels, the RLCG or S parameters, etc. The output layer consists of $n$ neurcns, each representing one of the elements in the $\mathbf{Y}$ vectur. The output of a neuron varies between 0 and 1, which are the asymptotic limits of the sigmoid transfer function, as given in Equation (4.5). Hence the entire output space must be scaled to vary between 0.1 and 0.9 , so that the values of the output remain within the active region of the sigmoid. 


\section{The Hidden Layer}

The number of neurons in the hidden layer is taken to be $p$, the choice of the value of $p$ being dictated by the complexity of the problem. A highly complex input-output relationship would require a higher number of neurons in the hidden layer, as each additional neuron provides several additional degrees of freedom during mapping. However, a neural network with a large value of $p$ requires a larger training time, and would unnecessarily increase the size $\mathcal{S}$ of the model, measured as the total number of weights and biases in the neural network:

$$
\mathcal{S}=p(m+1)+n(p+1)
$$

So as small a $p$ as would allow for correct mapping of the input/output relationship is used.

\subsubsection{Training}

The training of the neural network, as described earlier, is the process during which the neural network learns the relationship $\mathcal{F}$ between the input and output samples presented to it. This relationship is learned over several training epochs, in which a large set of input/output data is repeatedly presented to the neural network. The weights and biases in Equations 4.4-4.7 are adjusted such that the error between the outputs as predicted by the neural network and the outputs of the training set is minimized. The algorithm employed is based on the classical multi-layered backpropagation algorithm, and is described as follows.

For a given set of input data, say $a_{k}, k=1,2, \ldots Q$ whose corresponding output set is $d_{k}$, if the neural network network predicts the outputs to be $y_{k}$, the batch-mode 
backpropagation error $E_{a v}$ is defined as

$$
E_{a v}^{\prime}=\sum_{k=1}^{Q} E_{k}=\frac{1}{Q} \sum_{k=1}^{Q}\left[\frac{1}{2} \sum_{j=1}^{n}\left(y_{k j}-d_{k j}\right)^{2}\right]
$$

where $E_{k}$ represents the individual mean-squared error of the $k$ th sample. $E_{a v}$ is the error to be minimized during training. After each training epoch, during which the set of $Q$ data points is presented to the network, this error is determined, and the weights and biases are updated in the general direction of error minimization. The update equations for the $t^{\text {th }}$ epoch, with momentum $\alpha$ and learning rate $\eta$ are

$$
\begin{aligned}
v_{h j}^{t+1} & =v_{h j}^{t}-\eta\left(E_{a v}^{t}\right) \cdot \frac{\partial E_{a v}^{t}}{\partial v_{h j}}+\alpha \cdot\left(v_{h j}^{t}-v_{h j}^{t-1}\right) \\
w_{i h}^{t+1} & =w_{i h}^{t}-\eta\left(E_{a v}^{t}\right) \cdot \frac{\partial E_{a v}^{t}}{\partial w_{i h}}+\alpha \cdot\left(w_{i h}^{t}-w_{i h}^{t-1}\right) \\
b_{h}^{t+1} & =b_{h}^{t}-\eta\left(E_{a v}^{t}\right) \cdot \frac{\partial E_{a v}^{t}}{\partial b_{h}}+\alpha \cdot\left(b_{h}^{t}-b_{h}^{t-1}\right) \\
c_{j}^{t+1} & =c_{j}^{t}-\eta\left(E_{a v}^{t}\right) \cdot \frac{\partial E_{a v}^{t}}{\partial c_{j}}+\alpha \cdot\left(c_{j}^{t}-c_{j}^{t-1}\right)
\end{aligned}
$$

The error sensitivities in the above equations are calculated using the following equations.

$$
\begin{aligned}
\frac{\partial E_{a v}}{\partial v_{h j}} & =\frac{\partial}{\partial v_{h j}}\left(\frac{1}{2 Q} \sum_{k=1}^{Q} \sum_{j=1}^{n}\left(y_{j k}-d_{j k}\right)^{2}\right) \\
& =-\frac{1}{Q} \sum_{k=1}^{Q}\left(y_{j k}-d_{j k}\right) \cdot y_{j k} \cdot\left(1-y_{j k}\right) \cdot z_{h k} \\
& =-\frac{1}{Q} \sum_{k=1}^{Q} \delta_{j k}^{(0)} \cdot x_{h k}
\end{aligned}
$$

where the term $\delta_{j k}^{(0)}$, given by,

$$
\delta_{j k}^{(0)}=\left(y_{j k}-d_{j k}\right) \cdot y_{j k} \cdot\left(1-y_{j k}\right)
$$


represents the local gradients at the $j$ th neuron in the output layer for the $k$ th sample.

$$
\begin{aligned}
\frac{\partial E_{a v}}{\partial w_{i h}} & =\frac{\partial}{\partial w_{i h}}\left(\frac{1}{2 Q} \sum_{k=1}^{Q} \sum_{j=1}^{n}\left(y_{j k}-d_{j k}\right)^{2}\right) \\
& =-\frac{1}{Q} \sum_{k=1}^{Q} z_{h k} \cdot\left(1-z_{h k}\right) \sum_{j=1}^{n}\left(y_{j k}-d_{j k}\right) \cdot \delta_{j k}^{(0)} \cdot a_{i k} \\
\frac{\partial E_{a v}}{\partial c_{j}} & =\frac{\partial}{\partial c_{j}}\left(\frac{1}{2 Q} \sum_{k=1}^{Q} \sum_{j=1}^{n}\left(y_{j k}-d_{j k}\right)^{2}\right) \\
& =-\frac{1}{Q} \sum_{k=1}^{Q}\left(y_{j k}-d_{j k}\right) \cdot y_{j k} \cdot\left(1-y_{j k}\right) \\
\frac{\partial E_{a v}}{\partial b_{h}} & =\frac{\partial}{\partial b_{h}}\left(\frac{1}{2 Q} \sum_{k=1}^{Q} \sum_{j=1}^{n}\left(y_{j k}-d_{j k}\right)^{2}\right) \\
& =-\frac{1}{Q} \sum_{k=1}^{Q} z_{h k \cdot}\left(1-z_{h k}\right) \sum_{j=1}^{n}\left(y_{i} \cdot-d_{j k}\right) \cdot \delta_{j k}^{(0)}
\end{aligned}
$$

The parameter, $\eta$, known as the learning rate is an important training parameter representing the step size of the error convergence process. A small value of $\eta$ affords stability but increases training time, while a large value of $\eta$ decreases the stability of the training process. $\eta$ is shown in the update equations as being a function of the backpropagation error $E^{t}$, meaning it is normally adaptive in nature, assuming a small value when far away from the solution, during which the value of $E^{t}$ is large, and a relatively larger value when closer to the solution, i.e. when the backpropagation error is small. The value of $\eta$ is normally taken to be between 0.1 and 0.5 .

The term $\alpha$ in the update equations is called the momentum, and adds an inertial element to the training process, ensuring that the update occurring is a fraction of the 
previous change. This helps avoiding local minima on the srror surface. $\alpha$ normally assumes a positive value lese than 1 .

Using the above equations, the backpropagation training algorithm can be summarized in the following steps:

Step 1. Initialization Seleci random initial values for the weights and biases $w_{i h}$, $b_{h}, v_{h j}$, and $c_{j}$ lying in the range of -0.5 and 0.5 .

Step 2. Presentation Present the training set $x_{k}, k=1,2, \ldots Q$ to the neural network.

Step 3. Forward Pass Compute the corresponding neural network output vector $y_{k}, k=1,2, \ldots Q$ using Equations 4.4-4.7.

Step 4. Update Compute the batch backpropagation error from Eq. 4.9, the error sensitivities using Equations 4.14-4.18, and update the weights using Equations 4.10-4.13.

Step 5. Termination Condition Check If $E^{t}$ is less than a specified tolerance value end training, else update learning rate (if necessary) and return to Step 2.

A more detailed description of the training algorithm can be had from Haykin's book [61].

For the applications in this thesis, the Matlab implementation of the backpropagation algorithm [95] is employed. 


\subsubsection{Data Generation}

In order to train and validate the neural network model, two sets of data are required. The first set, namely the training set, is a set of data points representing randomly chosen inputs to the model and their corresponding outputs. The number of data points needed in the training set is dependent upon the following

- the dimensionality of the problem, particularly the dimensionality of the input space

- the complexity of the relationship between the input and the output

- the size $S$, of the network.

Though a larger training set would give a better representation of the input-output space to be modeled, too large a training set would result in a needless increase in the time invested in data generation and training. Hence as small a data set as would fairly represent the entire range of operation of the model should be used.

The training set data is obtained through repeated off-line simulation using an accurate simulation technique, either an electrical CAD tool or an EM field solver such as those previously described, as the case may require. The simulator is repeatedly called, each time with a netlist incorporating input variables randomly chosen from the input space. A flow chart representing the data generation procedure is shown in Fig. 4.3. Two files of data are thus obtained, the input vector and the corresponding output vector, which constitute the training set. The training set can also be obtained as a collection of data from actual measurements, or from a memory look-up table if it is already available. 


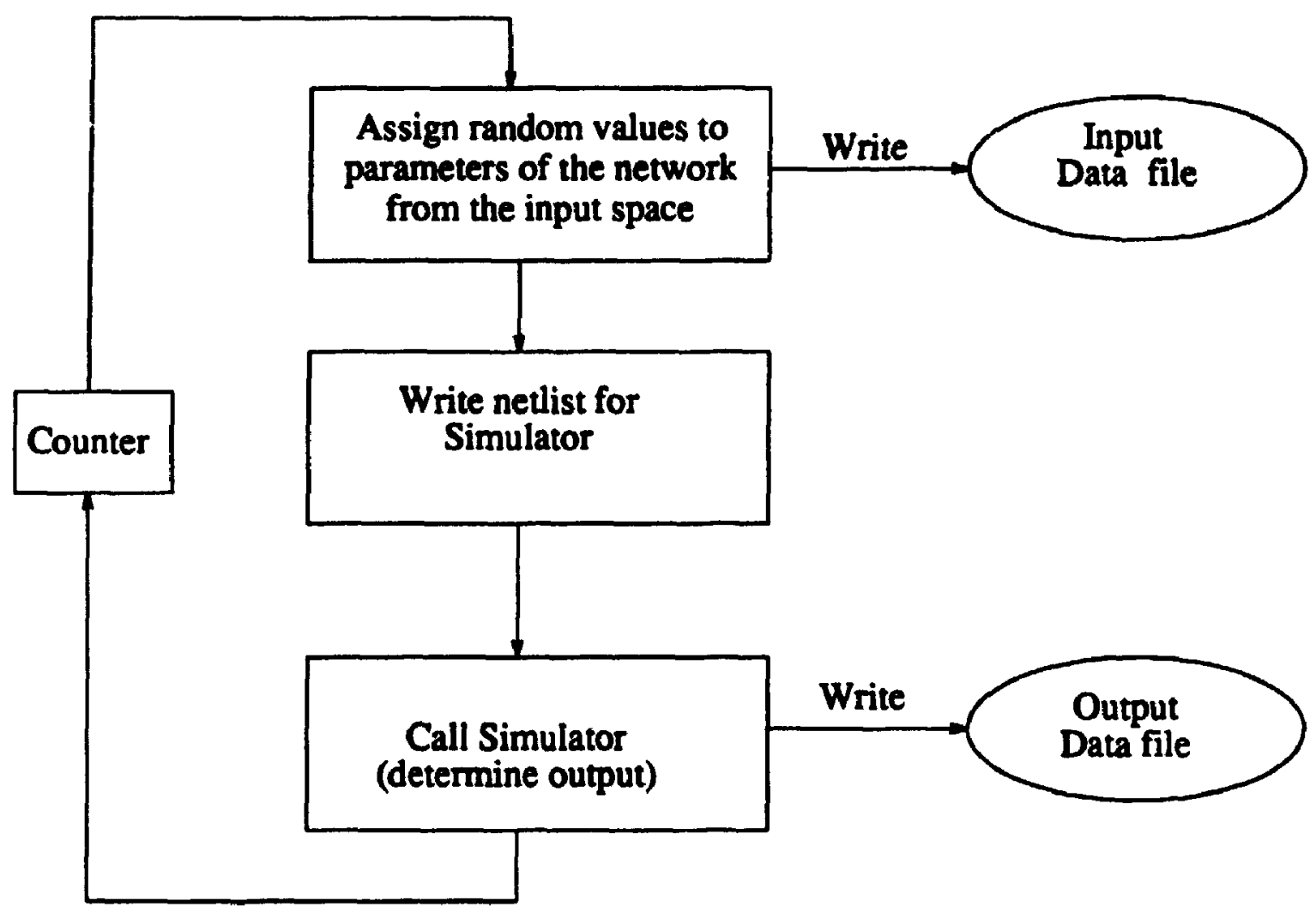

Figure 4.3: Algorithm used for generation of training and test data 
The second set of data, called the test set, is obtained in a manner identical to that described above and is used to test the accuracy of the model during and after training. The test set should be large enough to be representative of the entire input space, and its contents should be different from those of the training set.

\subsubsection{Model Validation}

The model is validated at the end of training, as well as periodically during the training process with both the training set and the test set. The rms error for the $k$ th sample of the training or test set is defined as

$$
\varepsilon_{k}=\left[\frac{1}{n} \sum_{i=1}^{n}\left(\frac{y_{k i}-d_{k i}}{d_{i}}\right)^{2}\right]^{\frac{1}{2}}
$$

where $n$ is the number of outputs of the neural network, $y_{k i}, i=1 \ldots n$ are the outputs as given by the neural network for the $k$ th sample, $d_{k i}$ is the corresponding actual output of the simulator, and $\bar{d}_{i}$ is the arithmetic mean of the absolute values of all ith output points over the entire space:

$$
\bar{d}_{i}=\frac{1}{Q} \sum_{k=1}^{Q}\left|d_{k i}\right|
$$

The average (test/training) error of validation is given as the arithmetic mean of the individual errors over the (test/training) set, i.e.

$$
\text { Average Error }=\frac{1}{Q} \sum_{k=1}^{Q} \varepsilon_{k}
$$

where $Q$ is the total number of samples in the set. It should be noted that the validation error is different from the backpropagation error $E_{a v}$ given in Equation (4.9), which is only used in the backpropagation training algorithm. 
The average test error is a good indication of how well the neural network has learned the relationship $\mathbf{Y}=\mathcal{F}(\mathbf{X})$. The neural network is validated periodically during training with the test set to ensure that the network is not over-learning, which is when the mapping is being concentrated too much on the specific data points of the training set rather than the general function. When over-learning starts, the average test error begins to increase though the average training error and backpropagation error continue to decrease.

Once developed, the model can be used to accurately calculate the desired output for any given set of inputs.

It can be seen that the neural network model as discussed above is independent of

- the actual input parameters chosen and the structure

- the output parameters

- the method used to obtain data. Measured data could be used, or a simulator of any degree of accuracy can be used.

It can thus be used as a generic CAD tool for modeling any kinds of parameters or structure, so long as there is a relationship between the input and output data. 


\subsection{Summary}

In this chapter, the backpropagation neural network model and the method that is adopted to construct high frequency CAD models have been discussed. This method is general enough to be adaptable to most CAD problems, and are employed in subsequent chapters to develop models for various applications. 


\section{Chapter 5}

\section{Neural Networks in Modeling Interconnects}

\subsection{Introduction}

In this chapter, the application of neural networks to the modeling of coupled interconnects and multi-conductor transmission line configurations is described. As discussed in Section 2.2.2, the modeling of interconnects connotes the determination of intrinsic parameters such as the RLCG parameters based on its physical and geometrical properties. Interconnect modeling encompasses a large class of problems in interconnect analysis, and is a necessary part of any problem where, due the frequency of operation, the RLCG parameters cannot be assumed to be constant. Different interconnect configurations are modeled using the general model developed in the previous chapter. A comparison of the performance of the neural network model and currently employed modeling techniques is made and the results discussed. 


\subsection{Example 1: Three Symmetric Microstrips}

In the first example, a configuration of three identical coupled interconnects running parallel to each other, the cross-section of which is shown in Fig. 5.1, is modeled for its frequency-dependent $\mathrm{L}$ and $\mathrm{C}$ parameters.

\subsubsection{Deflnition of Input/Output Parameters}

The input parameters of the model are chosen to be those which would typically vary during design optimization, as well as the frequency, as the RLCG parameters in general are frequency-dependent. Hence the inputs to the model are the thickness and width of the interconnects, the height of the dielectric, the separation of the interconnects (which is the distance between the centres of the conductors), and the frequency of operation. In the symmetric case, the width of the three conductors is the same, and the separation between adjacent conductors is the same.

The input vector is defined mathematically as

$$
\mathbf{X}=(W, t, s, h, \omega)
$$

Table 5.1: Input pa:ameters and their range (Example 1)

\begin{tabular}{|l|l|r|r|}
\hline Parameter & Symbol & Minimum & Maximum \\
\hline Conductor Width & $W$ & $5 \mathrm{mil}$ & $11 \mathrm{mil}$ \\
Conductor Thickness & $t$ & $0.7 \mathrm{mil}$ & $2.8 \mathrm{mil}$ \\
Separation & $s$ & $1 \mathrm{mil}$ & $16 \mathrm{mil}$ \\
Dielectric height & $h$ & $5 \mathrm{mil}$ & $1 \mathrm{nmil}$ \\
Frequency & $\omega$ & $1 \mathrm{MHz}$ & $8 \mathrm{GHz}$ \\
\hline
\end{tabular}

The ranges of the individual input variables are given in Table 5.1. The input paramters are all scaled to vary within the range of 0 and 1 linearly, with the exception 
of frequency, which is scaled logarithmically.

The outputs of the model are the elements of the $\mathbf{L}$ and $\mathbf{C}$ matrices, i.e.,

$$
\mathrm{L}=\left(\begin{array}{lll}
L_{11} & L_{12} & L_{13} \\
L_{21} & L_{22} & L_{23} \\
L_{31} & L_{32} & L_{33}
\end{array}\right)
$$

and

$$
\mathrm{C}=\left(\begin{array}{lll}
C_{11} & C_{12} & C_{13} \\
C_{21} & C_{22} & C_{23} \\
C_{31} & C_{32} & C_{33}
\end{array}\right)
$$

The total number of $\mathrm{L}$ and $\mathrm{C}$ paraneters to be simulated can thus be seen to be 18. But since the interconnects are identical in physical dimension and have symmetric cross-sections, we need to consider only the self-inductance $\left(L_{11}\right)$, two mutual inductances ( $L_{12}$ and $\left.L_{13}\right)$, the self-capacitance $\left(C_{11}\right)$, and two mutual capacitances $\left(C_{12}\right.$ and $\left.C_{13}\right)$, from which the entire $\mathbf{L}$ and $\mathbf{C}$ matrices can be constructed. Hence,

$$
\mathbf{Y}=\left(L_{11}, L_{12}, L_{13}, C_{11}, C_{12}, C_{13}\right)
$$

The $\mathbf{R}$ and $\mathbf{G}$ inatrices of an interconnect configuration wou'd be treated in a manner identical to that of the $\mathbf{L}$ and $\mathbf{C}$ parameters.

\subsubsection{Construction of the Model}

The neural network model is implemented with features as shown in Table 5.2. This table shows the number of inputs, outputs, and the number of hidden neurons used in the model, and gives information about the training and test processes.

The program used to generate the data is an EM field solver, SALI [93], which is based on modeling under the quasi-TEM assumption. However, any EM technique can be adopted. Training proceeded on a Sun workstation, the test error continually 
Table 5.2: Features of the neural network for the three symmetric multi-conductor model

\begin{tabular}{|l|r|}
\hline Feature & Value \\
\hline Number of inputs $(m)$ & 5 \\
Number of outputs to overall model & 18 \\
Number of outputs to neural net $(n)$ & 6 \\
Number of neurons in hidden layer $(p)$ & 10 \\
Size of model (in floating point numbers) & 126 \\
Size of training set & 500 \\
Size of test set & 500 \\
Data Generation Technique & Quasi-TEM Simulation \\
Training time (in hours) & 6 \\
Average training error & 0.0162 \\
Average test error & 0.0174 \\
\hline
\end{tabular}

decreasing until it leveled off at about 0.017 . The average training error as training proceeded is plotted against time in Fig. 5.2. The average test error was 0.0174 at the end of training.

The neural network model was found to estimate the $L$ and $C$ parameters with an accuracy comparable to the field solver used. The average test error in the results when the $\mathrm{L}$ and $\mathrm{C}$ parameters were modeled for 100 random circuit configurations from the test set are plotted in Fig. 5.3. Fig. 5.4 shows one of the outputs, $C_{13}$, plotted against a varying width of separation, $s$, as estimated by the neural network model and the EM field solver. 


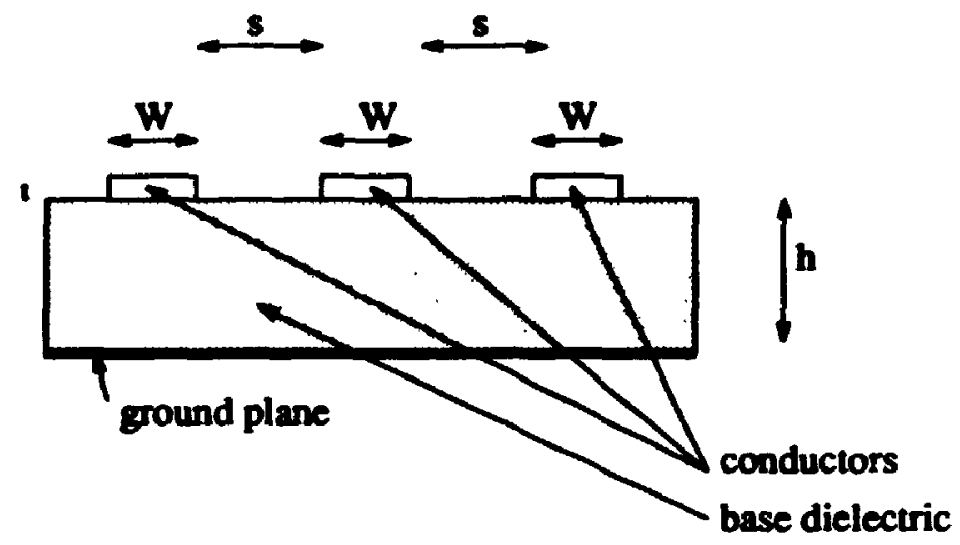

Figure 5.1: Cross-section of three microstrip lines

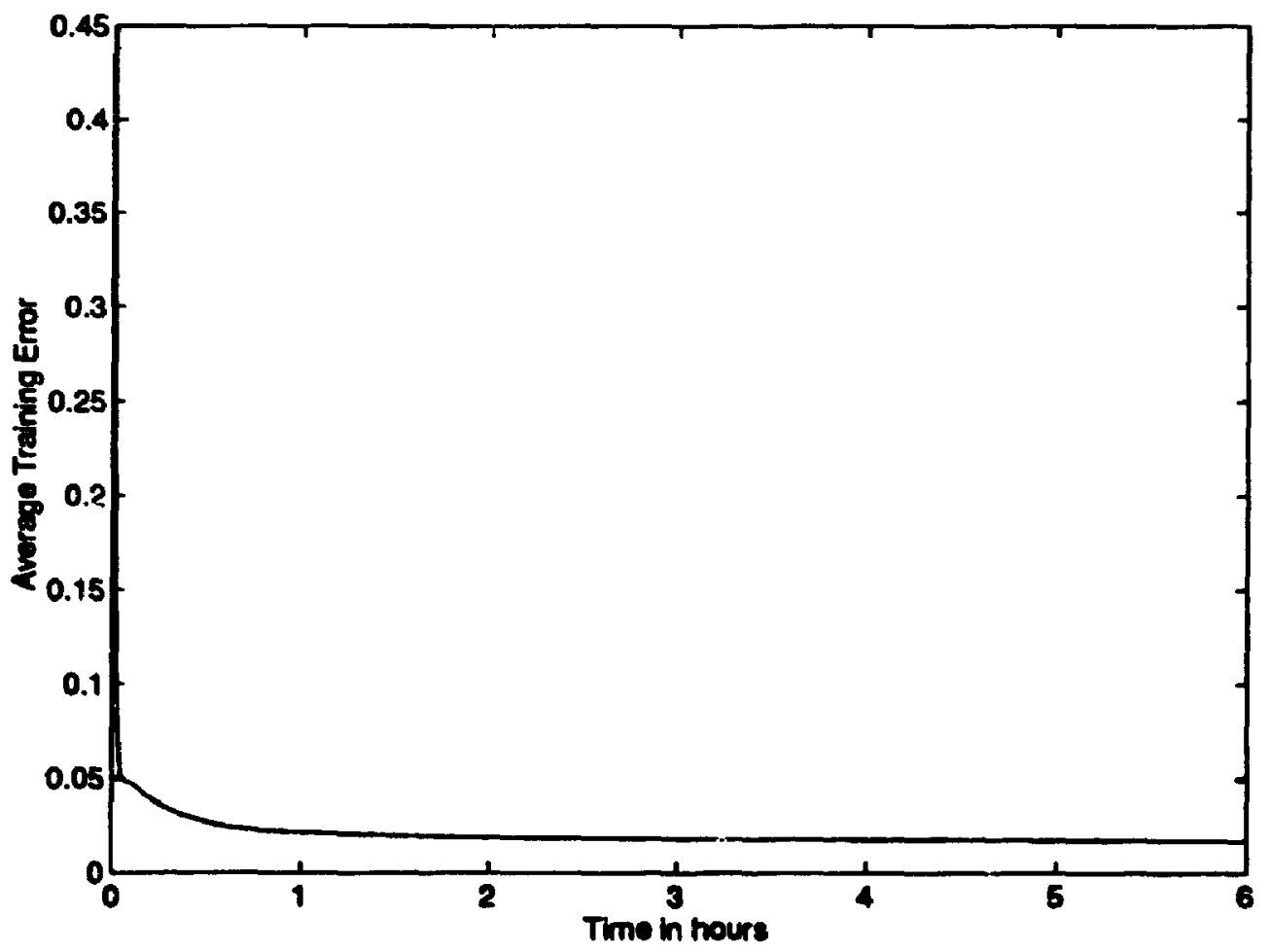

Figure 5.2: A verage training error as training proceeded for Example 1 


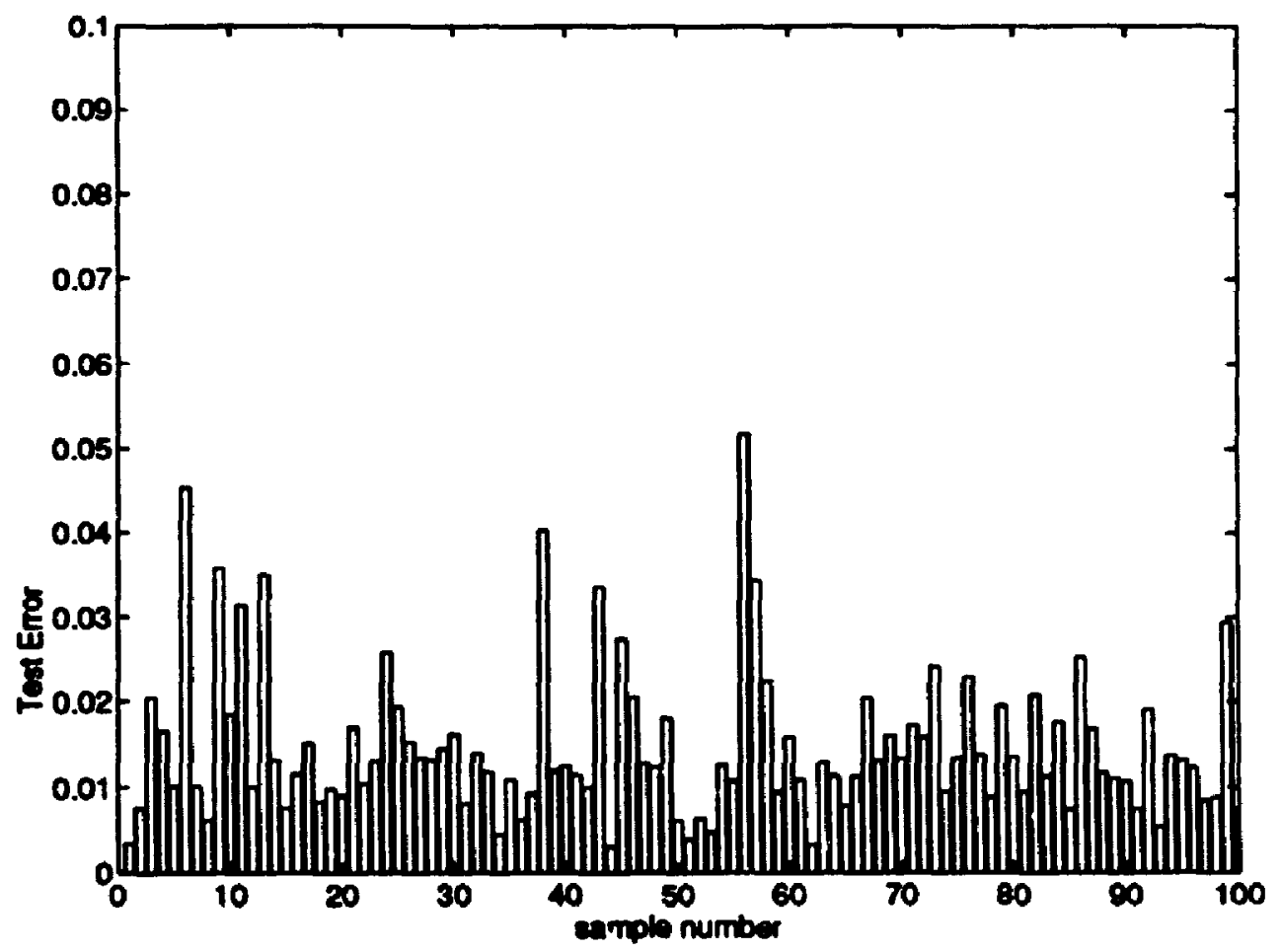

Figure 5.3: Test error for 100 random configurations for the three conductor model 


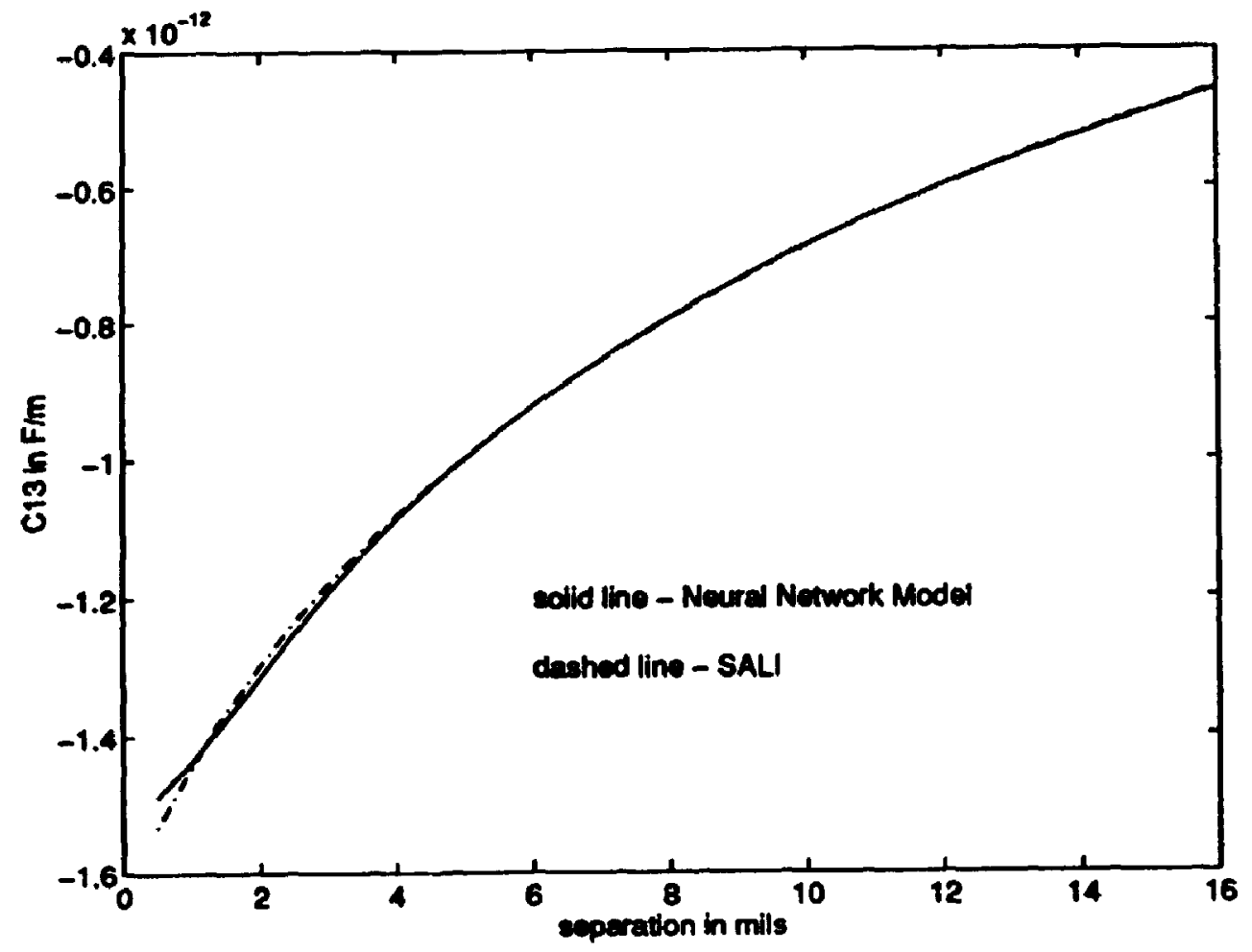

Figure 5.4: As a function of separation, parameter $C_{13}$ 


\subsection{Example 2: Two Asymmetric Microstrips}

The two asymmetric microstrip conductor configuration, as shown in Fig. 5.5, is similar to the previous example except that there are only two conductors, and these conductors differ in their width.

\subsubsection{Identification of Input/Output Parameters}

The input variables are identical to those in the previous example except that the width of the two conductors must be defined separately:

$$
\mathbf{X}=\left(W_{1}, W_{2}, t, s, h, w^{\prime}\right.
$$

Their ranges are as given in Table 5.1. As in the previous example, SALI is employed to generate data. There are eight outputs, namely the elements of the $\mathbf{L}$ and $\mathbf{C}$ matrices:

$$
Y=\left(L_{11}, L_{12}, L_{21}, L_{22}, C_{11}, C_{12}, C_{21}, C_{22}\right)
$$

\subsubsection{The Two Conductor Neural Network Model}

Table 5.3 shows the features of the neural network developed. The accuracy of the neural network model was in the range of the EM field solver, this being indicated in Fig. 5.6, which shows the test error of 100 random sample inputs from the test set. 


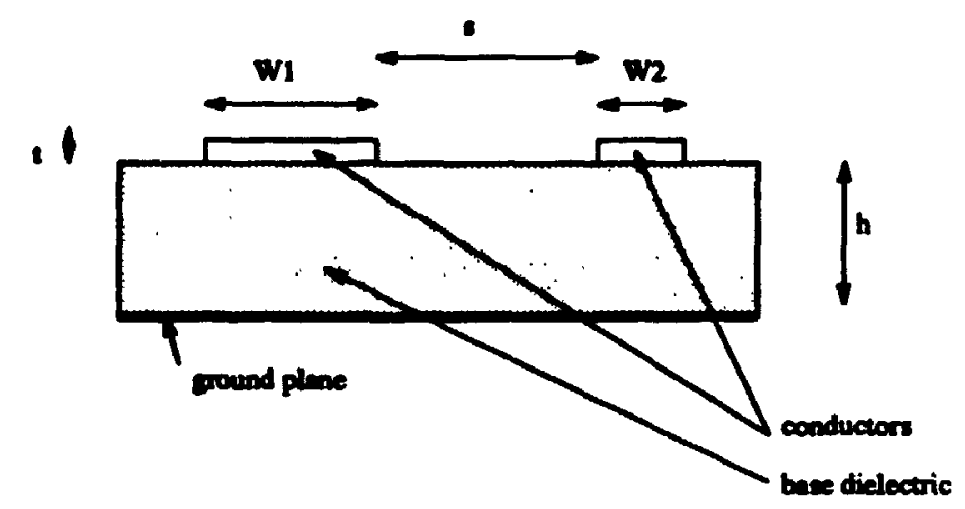

Figure 5.5: Two asymmetric microstrip lines

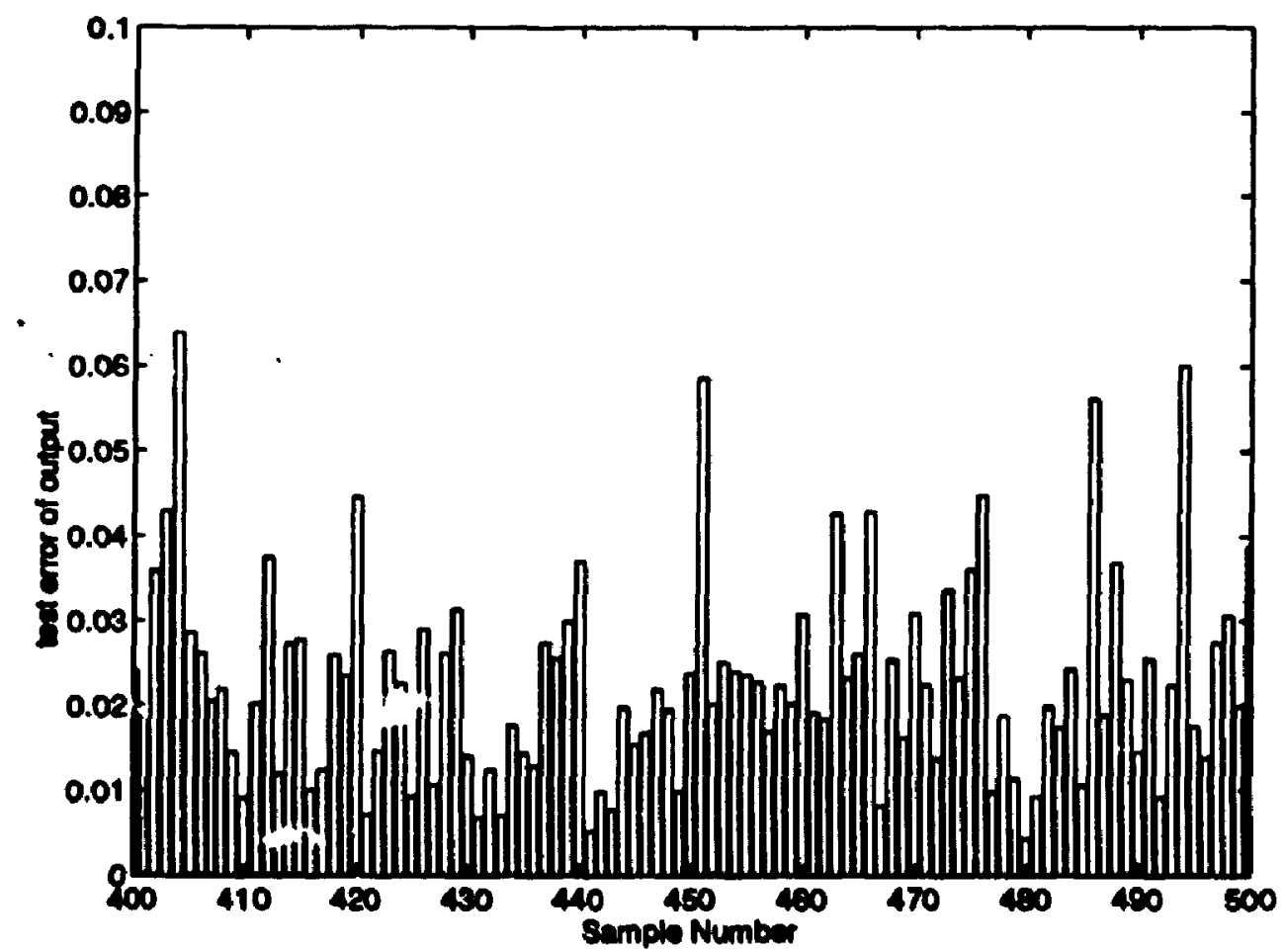

Figure 5.6: Test error for 100 random inputs from the test set for Example 2 
Table 5.3: Features of the neural network model for Example 2

\begin{tabular}{|l|r|}
\hline Feature & Value \\
\hline Number of inputs $(m)$ & 6 \\
Number of outputs to overall model & 8 \\
Number of outputs to neural net $(n)$ & 8 \\
Number of neurons in hidden layer $(p)$ & 10 \\
Size of model (in floating point numbers) & 158 \\
Training time (in hours) & 7 \\
Size of training set & 500 \\
Size of test set & 500 \\
Data Generation Technique & SALI \\
Average training error & 0.0233 \\
Average test error & 0.0253 \\
\hline
\end{tabular}




\subsection{Example 3: An 8-bit Data Bus (Eight Inter- connects in Parallel)}

In this model an 8-bit bus configuration as shown in Fig. 5.7, the cross-sectional view of which is shown in Fig. 5.8 is modeled.

In the region on a Printed Circuit Board between the I/O pad and the IC pins to which they are connected, eight interconnects run parallel to each other. This is a region of high crosstalk and noise due to the eight parallel conductors, and is thus especially critical in layout design.

\subsubsection{Input/Output Parameters}

A digital bus is inherently symmetric in structure, and consists of conductors of identical physical cross-section. The inputs of the model are the thickness, width, separation, height of dielectric, and the frequency. Their ranges are exactly the same as in the first example, as given in Table 5.4.

Table 5.4: Input parameters and their range for the 8-bit data bus

\begin{tabular}{||l|l|r|r|}
\hline Parameter & Symbol & Minimum & Maximum \\
\hline Conductor Width & $W$ & $5 \mathrm{mil}$ & $11 \mathrm{mil}$ \\
Cunductor Thickness & $t$ & $0.7 \mathrm{mil}$ & $2.8 \mathrm{mil}$ \\
Separation & $s$ & $1 \mathrm{mil}$ & $16 \mathrm{mil}$ \\
Dielectric height & $h$ & $5 \mathrm{mil}$ & $10 \mathrm{mil}$ \\
Frequency & $\omega$ & $1 \mathrm{MHz}$ & $8 \mathrm{GHz}$ \\
\hline
\end{tabular}

The outputs modeled in this case are the elements of the $L$ and $C$ parameter matrices, each of which contains 64 elements. However, since the interconnects are identical in physical dimension, width of separation, and have symmetric cross-sections, the 8 by 8 matrices are symmetric about both diagonals. Thus the entire $L$ matrix can 


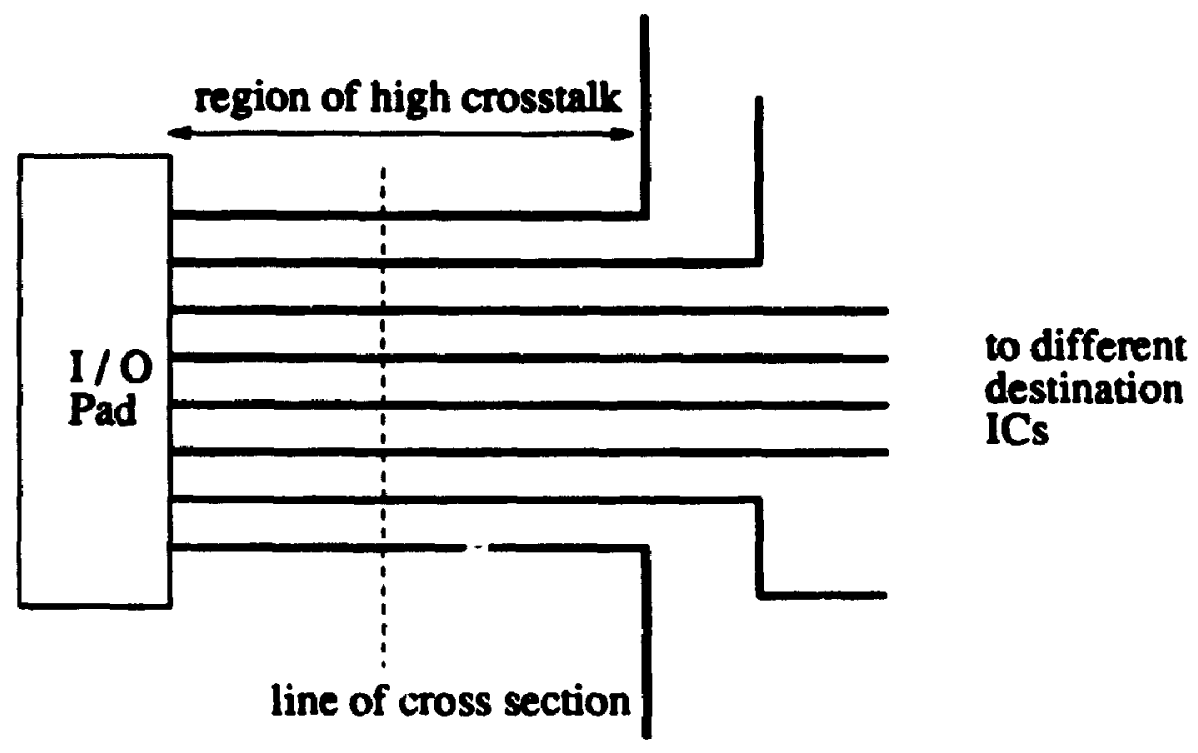

Figure 5.7: 8-bit bus on a PCB, showing region of high crosstalk and signal noise

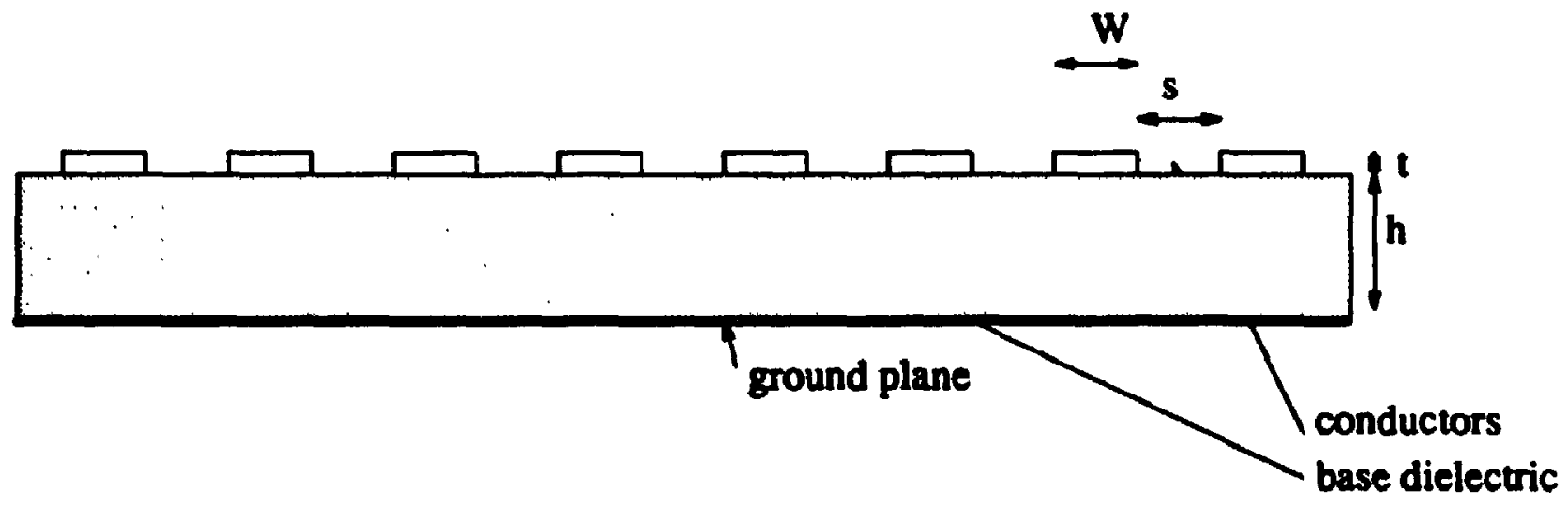

Figure 5.8: Cross-section of the 8-bit digital bus 
be constructed from one self-inductance $\left(L_{11}\right)$, and 16 mutual inductances. Similarly, the $\mathrm{C}$ matrix can be built from one self-capacitance $\left(C_{11}\right)$, and 16 values of mutual capacitance. Furthermore, in the case of the inductance matrix, the values of the mutual inductances of the elements farthest apart, namely $L_{18}, L_{17}, L_{28}$, and $L_{27}$ are extremely small, and hence are not modeled, but are set to a nominal average value. The $\mathbf{L}$ matrix is represented as

$$
\mathbf{L}=\left(\begin{array}{llllllll}
L_{11} & L_{12} & L_{13} & L_{14} & L_{15} & L_{16} & \bar{L}_{17} & \bar{L}_{18} \\
L_{12} & L_{11} & L_{23} & L_{24} & L_{25} & \bar{L}_{26} & \bar{L}_{27} & \bar{L}_{28} \\
L_{13} & L_{23} & L_{11} & L_{34} & L_{35} & L_{36} & \bar{L}_{37} & L_{38} \\
L_{14} & L_{24} & L_{34} & L_{11} & L_{45} & L_{35} & L_{25} & L_{15} \\
L_{15} & L_{25} & L_{35} & L_{45} & L_{11} & L_{34} & L_{24} & L_{14} \\
L_{16} & \bar{L}_{62} & L_{36} & L_{34} & L_{35} & L_{11} & L_{23} & L_{13} \\
\bar{L}_{71} & \bar{L}_{72} & \bar{L}_{73} & L_{24} & L_{25} & L_{23} & L_{11} & L_{12} \\
\bar{L}_{81} & \bar{L}_{82} & L_{16} & L_{14} & L_{15} & L_{13} & L_{12} & L_{11}
\end{array}\right)
$$

where the elements with bars are fixed at nominally average values and not separately modeled. The $\mathbf{C}$ matrix is similar to the $\mathbf{L}$ matrix, except that all parameters are modeled. Hence, the number of outputs for the neural network are $17 C$ elements and $13 L$ elements, giving a total of 30 .

\subsubsection{Construction of the Neural Network Model}

The main features of the neural network developed are tabulated in Table 5.5.

The model was capable of accurately predicting the $L$ and $C$ parameters. Fig. 5.9 shows the test error in 100 random inputs from the test set. Fig. 5.10 shows the values of the mutual capacitances in a given configuration as one moves away from the first cunductor on the left, i.e. the values on the first row of the $\mathbf{C}$ matrix. 


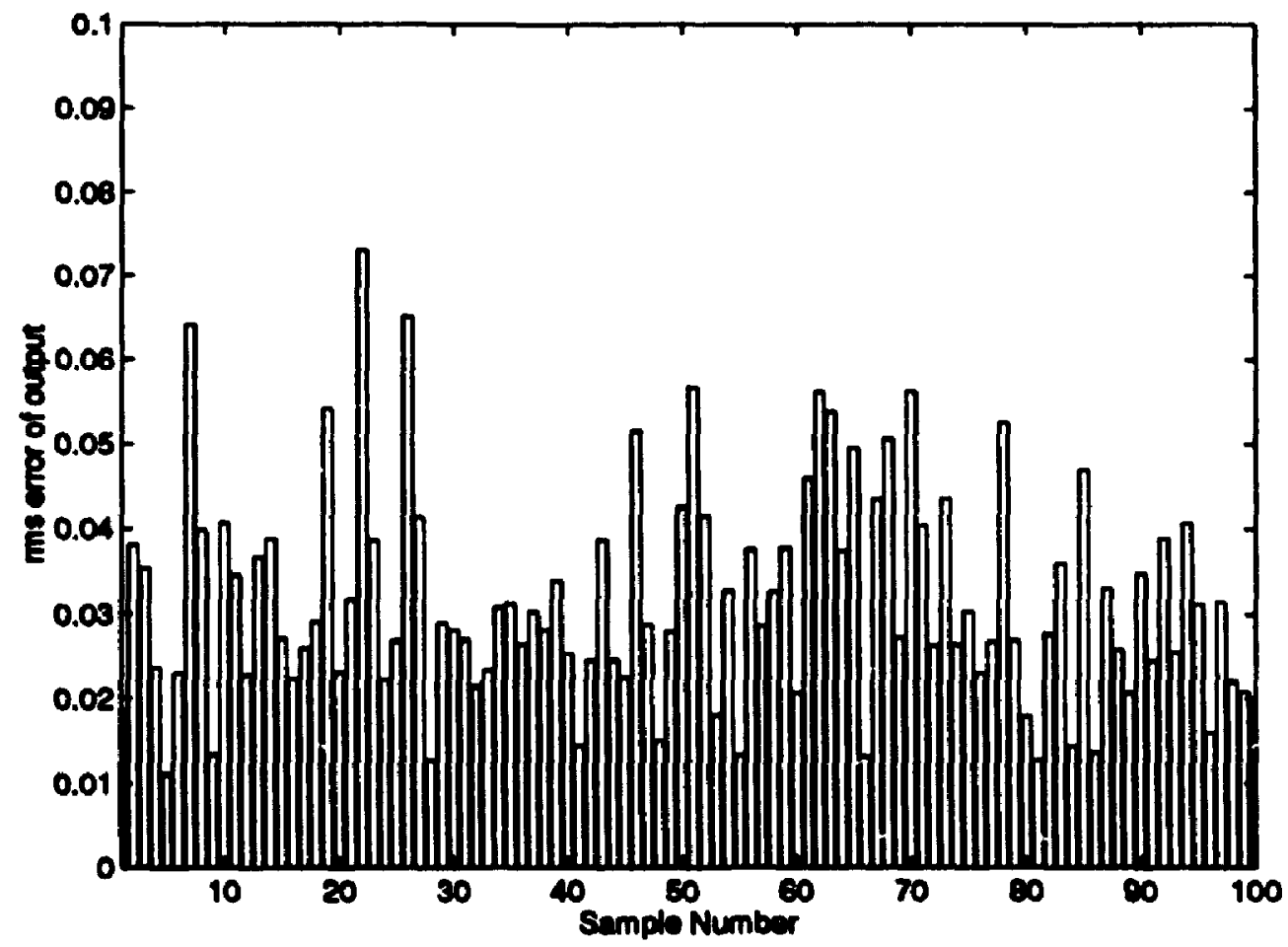

Figure 5.9: Test error over 100 random inputs for the 8-bit bus model 


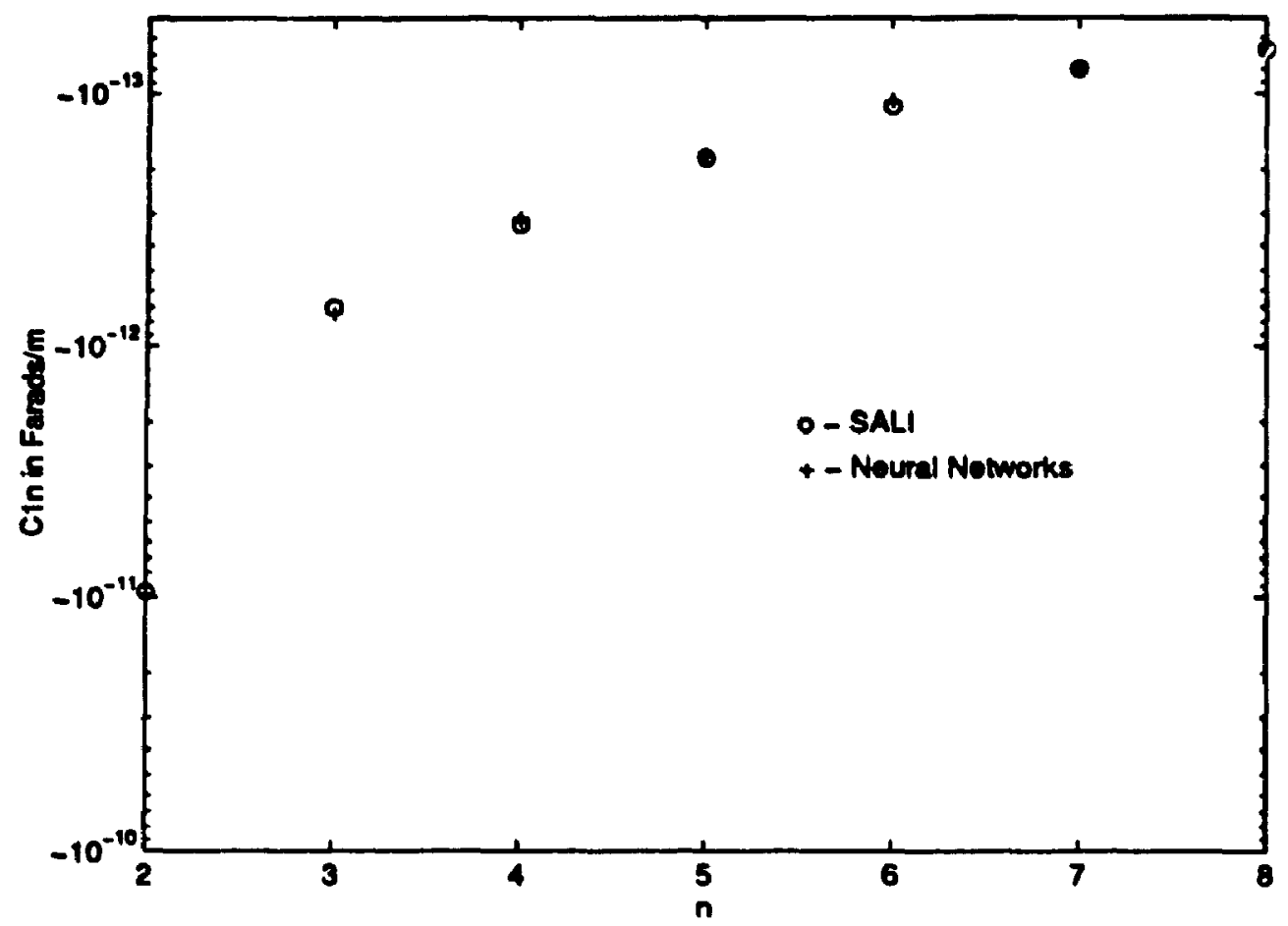

Figure 5.10: The values of mutual capacitances between the conductors of a given configuration of the 8-bit bus 
Table 5.5: Features of neural network model for Example 3

\begin{tabular}{|l|r|}
\hline Feature & Value \\
\hline Number of inputs (m) & 5 \\
Number of outputs to overall model & 128 \\
Number of outputs to neural net $(\mathrm{n})$ & 30 \\
Number of neurons in hidden layer $(\mathrm{p})$ & 10 \\
Size of model (in floating point numbers) & 390 \\
Training time (in hours) & 7 \\
Size of training set & 500 \\
Size of test set & 500 \\
Data Generation Technique & SALI \\
Average training error & 0.0348 \\
Average test error & 0.0351 \\
\hline
\end{tabular}

\subsection{Run-Time Comparison with an EM Field Solver}

The neural network model was compared with SALI in terms of run-time requirements. The run-times on a Sparc station10 for one simulation of the EM field solver, and for the neural network model are shown in Table 5.6.

Table 5.6: Comparison of CPU time requirements for neural network model and EM simulation

\begin{tabular}{|l|l|}
\hline Method & Run-time for 1 Simulation \\
\hline EM Field Solver & $8-10$ seconds \\
\hline NN Model & $5-6$ milliseconds \\
\hline
\end{tabular}

It can be seen that the neural network model offers a speed-up of three orders of magnitude. 


$$
2 \frac{2}{\text { of/de }} 2
$$




\subsection{Discussion of Results}

The above set of examples demonstrates the application of neural networks to modeling the frequency-dependent parameters of a coupled microstrip configuration.

It can be seen that a substantial speed-up over existing techniques is attainable. In all the examples, the neural network model was found to be three orders of magnitude faster than EM simulation. The low run-time can enable designers to incorporate electromagnetic methods for interconnect analysis: in iterative optimization techniques, without worrying too much about time constraints. Even several thousand calls of the model would only require several seconds of on-line CPU time, as opposed to existing techniques which would consume several hours.

Another important advantage of neural networ!:s made apparent by the above examples is their low memory requirements. A neural network can be developed for any defined cross-section which might be encountered during layout optimization, irrespective of the size or the number of conductors. Memory requirements are not high, and do not grow exponentially with the number of inputs or outputs added as a look-up table would. This is see. in the case of Example 2, where the input vector is one element larger than Example 1, or more dramatically in Example 3, where the output vector contains 5 times more elements than in Example 2, but the increase in the size of the model is less than directly proportional.

Neural networks offer a general solution to function mapping, irrespective of the range of the input variables. They are capable of mapping over a very wide range of input variation. In traditional approaches for modeling the frequency-dependent parameters, this is not the case, as different algorithms and simplifications are used in different regions, depending on the frequency. In the case of neural networks, a single 
model is sufficient to model frequency dependence over the entire range of variation.

\subsection{Summary}

In this chapter, the application of neural networks to the modeling of multiple interconnects has been described. The cases studied demonstrated the potential advantages of using neural networks over existing techniques for on-line CAD and optimization techniques. Neural network models were found to outperform existing techniques by a speed-up of three orders of magnitude, had low memory requirements, and were capable of handling large ranges of input variation. 


\section{Chapter 6}

\section{The Interconnect Network Simulator}

\subsection{Introduction}

The application of neural networks to problems involving the simulation of interconnection networks is discussed in this chapter. These problems are traditionally solved using electrical simulators such as those described in Section 2.4, and represent another important class of problems in interconnect analysis. The main difference between the nature of the models presented in this chapter and those of the previous chapter is that the output parameters modeled here are dependent not only on the properties of the interconnects but also on the circuit elements to which they are connected. In order to study the application of the neural network approach to interconnect network simulation, a interconnect network simulator is constructed and evaluated against existing techniques. 


\subsection{The Interconnect Network Simulator: A De- scription}

A typical high-speed circuit, such as a PCB or an MCM (multi-chip module) consists of numerous networks of interconrects which carry signals between the different components and devices comprising the circuit. Each individual network has a source pin connected to one or several destination pins. A typical interconnection network is depicted in Fig. 6.1. An interconnection network can be characterized by its size, i.e. the number of destination pins present, or the number of individual interconnects it comprises.

The signal integrity parameter of interest simulated here is propagation delay. Given a knowledge of the layout, the delays of a signal at the terminations of the interconnects are simulated. The electrical equivalent circuit of Fig. 6.1 is shown in Fig. 6.2.

For a given network size, the size of both the input vector and the output vector are constant, each increasing as the size of the network increases. As explicit definition of the input and output vectors are required for neural network implementation, it would be logical to design separate neural networks for each size of interconnect network expected.

The size of an interconnect network in a digital system does not increase indefinitely, as it is constrained by the fan-out of the source to which it is connected. A large number of output nodes would create too high a load on the source, resulting in reduced performance. Most typical digital pins would have four to five destination pins attached to it. Hence the interconnect network simulator should be capable of handling networks up to a size of 5 . The following sections in this chapter describe 


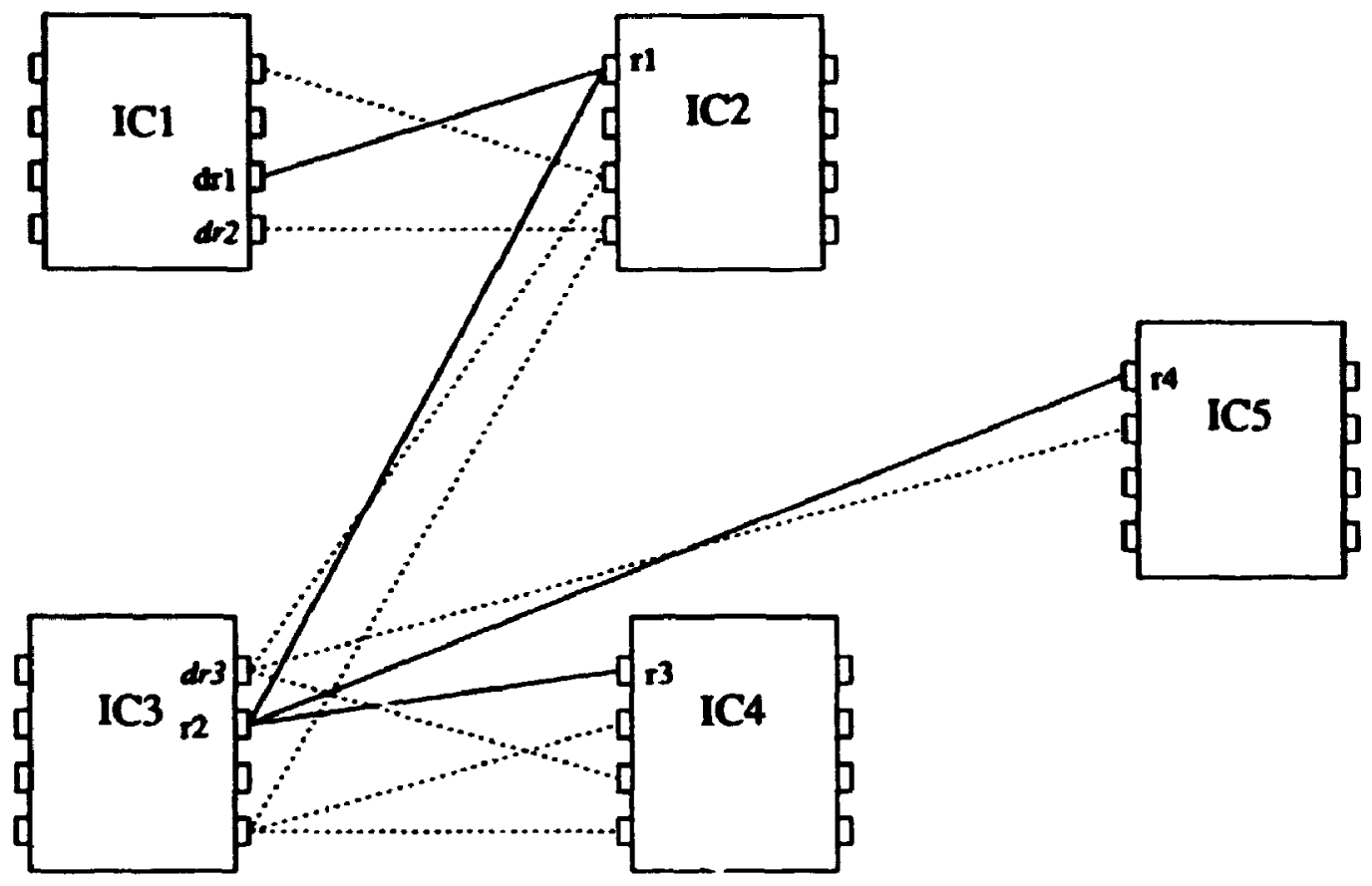

interconnections between different pins

\section{typical signal path}

dr1, dr2, dr3 - driver pins

$\mathrm{r} 1, \mathrm{r} 2, \ldots \mathrm{r} 4 \quad-$ receiver pins for signal from $\mathrm{dr} 1$

Figure 6.1: A typical interconnect network with IC pins connected by several interconnects 


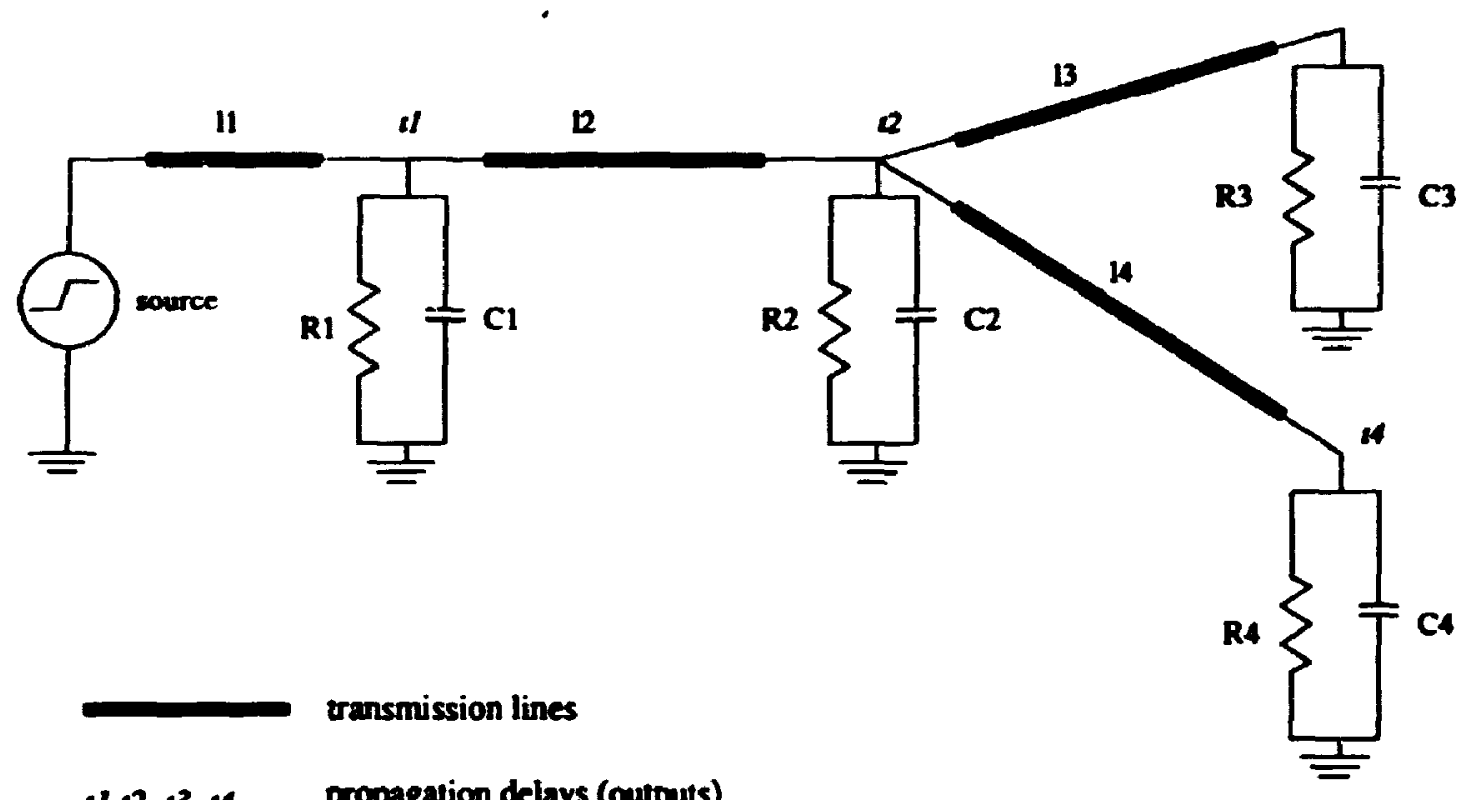

Figure 6.2: Electrical equivalent of interconnect configuration, showing the outputs of the model 
the construction of the neural networks constituting the simulator.

\subsubsection{An Interconnect Network: Design Parameters}

The design parameters available to a circuit designer and variables during layout are:

- input signal voltage

- input signal rise-time

- source impedance

- termination impedance (interconnect load)

- network topology

In a layout problem, the circuit topology is also available as a design variable. During layout optimization several different layouts of the board are configured and simulated to determine the optimal layout, as described in [9]. In traditional ap. proaches involving netlists, the topology of the interconnect network is not explicitly expressed. However, a neural network approach requires the explicit quantification of all design variables in the input vector. A method of quantifying network topology is described in the next section.

\subsubsection{Network Topology: A Design Variable}

Network topology is an important design variable available to a circuit layout designer. In order to use a neural network approach, explicit parameterization of the topology is required.

Given a certain number of interconnects in a network commencing from a given source, there are a specific number of configurations that it can take. Using graph 
theory, the source node can be considered the root of a set of nodes to be connected as a directed graph. Each possible circuit configuration thus forms a rooted tree, and the network topology is one of the possible rooted trees for a given number of nodes. If the nodes are numbered in a systematic way, the node numbers can be used to uniquely define the network topology. The following steps are used to number the nodes:

Step 1 The source node is numbered 1.

Step 2 The other nodes in the tree are numbered in a breadth-first manner

Step 3 On a layer with more than one node, the sibling node spawning the deepest descendant is is numbered first

Step 4 If there is a tie, the node with the larger number of children is numbered first.

After number the nodes in this manner, it is seen that the nodes which vary from configuration to configuration are those at which each interconnect commences (excluding the first interconnect, which always starts from the source). This is illustrated in Fig. 6.3, where all possible configurations of a network with four intercunnects are drawn and the node sets defining their topology given.

From the illustration it becomes apparent that the nodes farthest away from the source (which, on an average can be expected to have the largest propagation delay), have the highest numbers. Also, again in an average sense, a semblance of a relationship between the numbering of the nodes and its load in terms of circuit terminations can be perceived. 


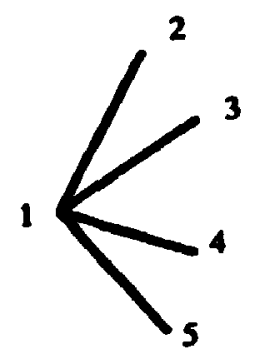

$(1,1,1)$

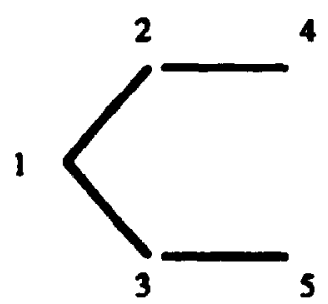

$\{1,2,3\}$

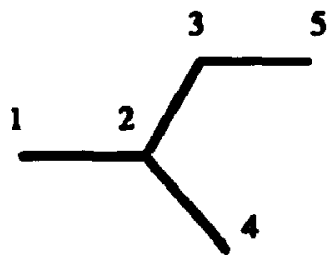

$(2,2,3)$

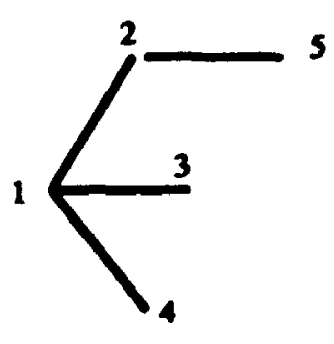

[1,1.2)

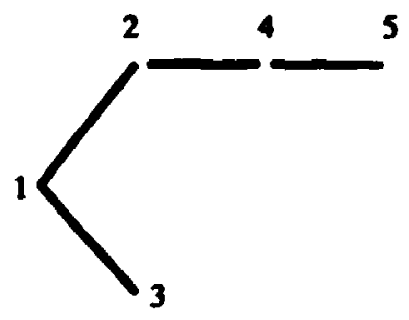

$[1,2.4]$

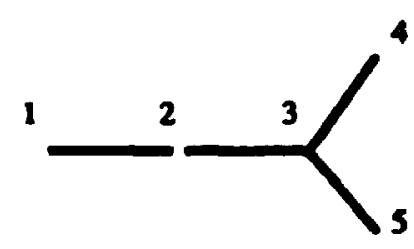

$(2,3,3)$

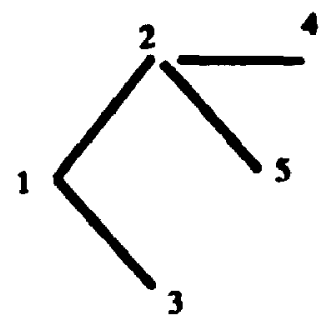

(1.2.2)

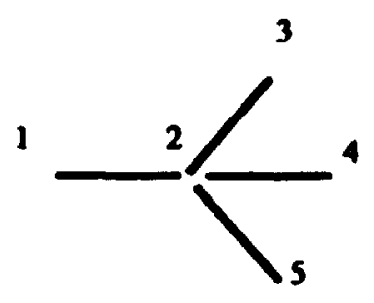

$[2,2,2\}$

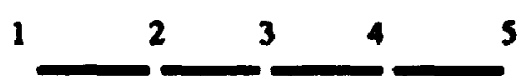

$(2,3,4)$

Figure 6.3: Possible network configurations for four nodes. The values of the variables defining the topology are also given 
The number of possible network configurations for a given number of interconnects is given in the following table ( 1 to 8 interconnects) [94]:

Table 6.1: Number of possible configurations for a given number of interconnects

\begin{tabular}{|c|c|}
\hline Number of Interconnects & Number of Configurations \\
\hline 1 & 1 \\
2 & 2 \\
3 & 4 \\
4 & 9 \\
5 & 20 \\
6 & 48 \\
7 & 115 \\
8 & 286 \\
\hline
\end{tabular}

As the output parameter of concern is the propagation delay at each node (in other words at the termination of each interconnect), for a given network of $\boldsymbol{n}$ interconnects, the output vector would be of dimension $n$. Hence for each value of $n$ that might be encountered during layout, a separate model must be constructed as described earlier.

\subsection{A Network of Three Interconnects}

In this example, the propagation delays of a signal propagating in a network of three interconnects on a PCB are simulated. The electrical equivalent circuit is shown in Fig. 6.4.

\subsubsection{Deflning the Input/Output Parameters}

The interconnect network, being of size 3 , indicates that there are three destination pins, and hence the output vector wound constitute three propagation delays. The variables available during design of the $\mathrm{PCB}$ are the driver (source) characteristics, 


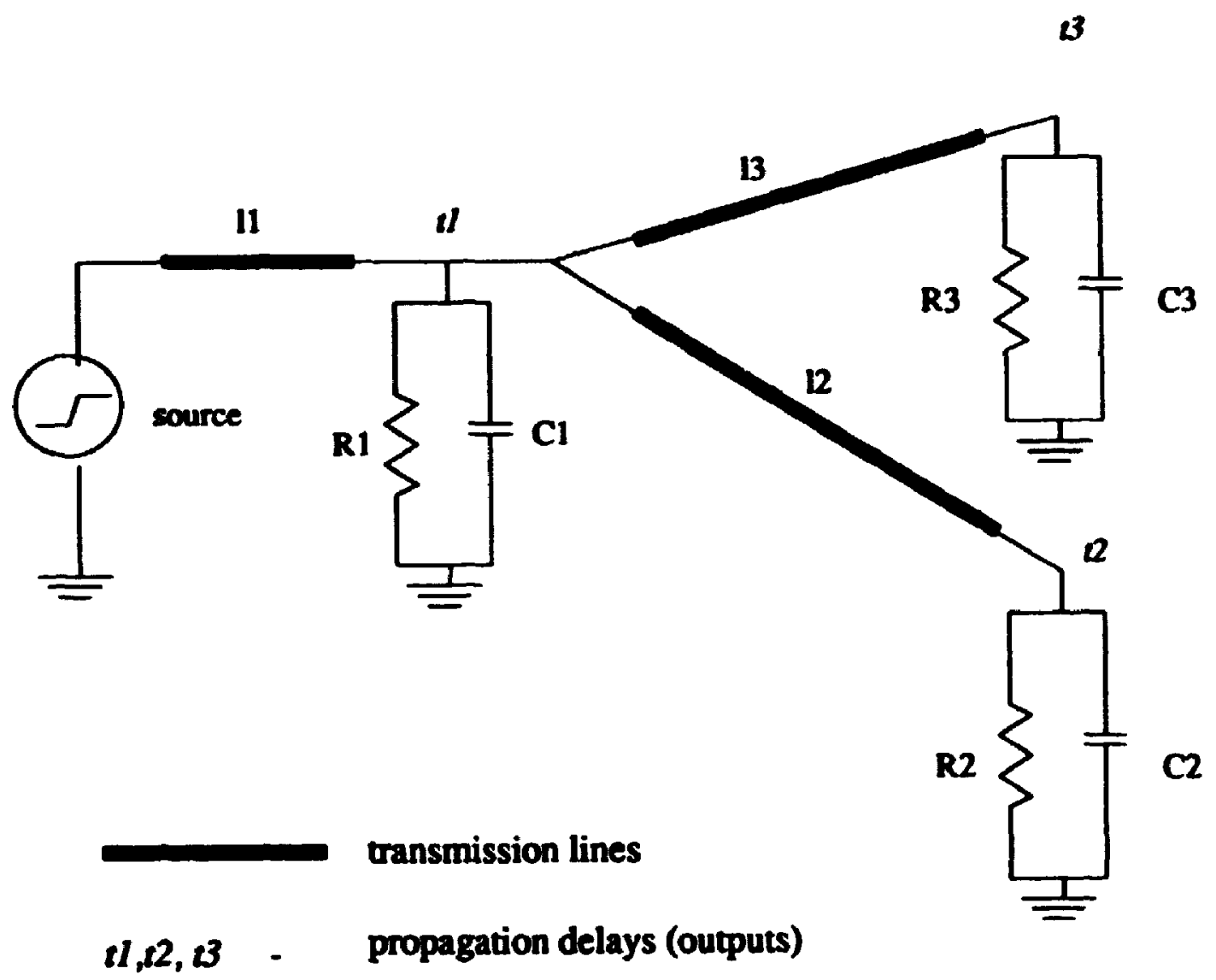

Figure 6.4: Electrical representation of a three interconnect network 
the receiver (load) characteristics, the lengths of the interconnects, and the network topology. The input variables to the problem are listed with their ranges in Table 6.2.

Table 6.2: Range of input parameters to the three interconnect network model

\begin{tabular}{|l|r|l|r|r|}
\hline Parameter & Number & Unit & Minimum & Maximum \\
\hline Interconnect length $(l)$ & 3 & $\mathrm{~cm}$ & 1 & 15 \\
Termination Resistance $\left(\boldsymbol{R}_{i}\right)$ & 3 & Ohms & 100 & 100,000 \\
Termination Capacitance $\left(C_{i}\right)$ & 3 & $\mathrm{nF}$ & 3.3 & 5 \\
Source Resistance $\left(R_{s}\right)$ & 1 & Ohms & 13.3 & 45 \\
Input Rise-time $\left(r_{t}\right)$ & 1 & $\mathrm{~ns}$ & 1.6 & 10 \\
Peak Voltage $\left(V_{p}\right)$ & 1 & $\mathrm{~V}$ & .8 & 5 \\
Source Edges (defining topology) & 2 & - & 1 & 3 \\
\hline
\end{tabular}

The input vector is

$$
\mathbf{X}=\left(l_{1}, R_{i}, C_{n}, R_{2}, r_{t}, V_{p}, e_{j}\right) \quad \text { for } \quad i=1,2,3 \quad \text { and } \quad j=1,2
$$

The outputs of the model are the propagation delays at the three terminations, propagation delay being measured as the time taken for the signal to reach $80 \%$ of its steady state value:

$$
\mathbf{Y}=\left(\tau_{i}\right) \quad \text { for } \quad i=1,2,3
$$

\subsubsection{Construction of the Model}

The number of inputs, outputs, and the number of hidden neurons chosen for the neural network are shown in Table 6.3.

Propagation delays are normally evaluated using and electrical analysis tool such as NILT [19], which uses a netlisted description of the circuit. This is thus the method 
Table 6.3: Features of three interconnect network model

\begin{tabular}{|l|r|}
\hline Feature & Value \\
\hline Number of inputs $(m)$ & 14 \\
Number of Outputs $(n)$ & 3 \\
Number of neurons in hidden layer $(p)$ & 20 \\
Size of model (in floating point numbers) & 363 \\
Size of training set & 2000 \\
Size of test set & 1000 \\
Data Generation Technique & NILT \\
Training time (in hours) & 36 \\
Average training error & 0.0298 \\
Average test error & 0.0325 \\
\hline
\end{tabular}

adopted to generate training and test data. However, any of the methods describrd in Section 2.4 can be used for data generation.

Training proceeded with a continuous decrease in both training and test error until it leveled off at about 0.0325 . The results of delay simulation for 100 random circuit configurations from the test set are plotted in Fig. 6.5.

\subsection{Delay Simulation in a Four Interconnect Net- work}

In this example, the size of the network is increased to four. Fig. 6.2 shows the equivalent circuit of one such network.

\subsubsection{The Inputs and Outputs}

The inputs are identical to the previous example, except that the number of inputs must increase to accommodate the extra interconnect and associated termination impedance. Table 6.4 shows this. 


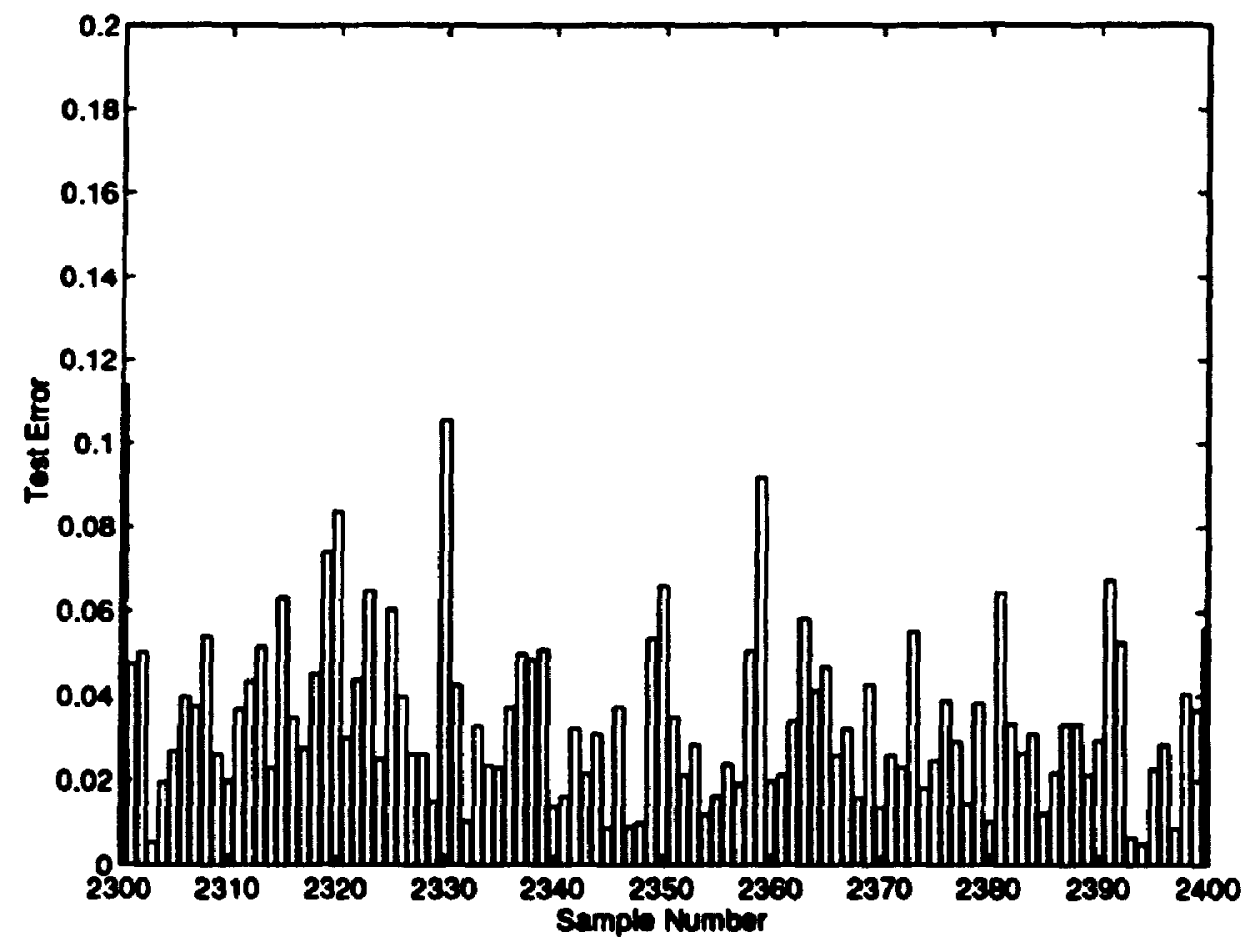

Figure 6.5: Error in 100 random test samples of the three interconnect network model 
Table 6.4: Range of input parameters for the four interconnect network model

\begin{tabular}{|l|r|l|r|r|}
\hline Parameter & Number & Unit & Minimum & Maximum \\
\hline Interconnect length $(l)$ & 4 & $\mathrm{~cm}$ & 1 & 15 \\
Termination Resistance $\left(R_{i}\right)$ & 4 & Ohms & 100 & 100,000 \\
Termination Capacitance $\left(C_{i}\right)$ & 4 & $\mathrm{nF}$ & 3.3 & 5 \\
Source Resistance $\left(R_{t}\right)$ & 1 & Ohms & 13.3 & 45 \\
Input Rise-time $\left(r_{t}\right)$ & 1 & $\mathrm{~ns}$ & 1.6 & 10 \\
Peak Voltage $\left(V_{p}\right)$ & 1 & $\mathrm{~V}$ & .8 & 5 \\
Source Edges (defining topology) & 3 & - & 1 & 5 \\
\hline
\end{tabular}

The input and output vector are mathematically defined as:

$\mathbf{X}=\left(l_{i}, R_{i}, C_{i}, R_{s}, r_{i}, V_{p}, e_{j}\right) \quad$ for $\quad i=1,2,3,4 \quad$ and $\quad j=1,2,3$.

and

$$
\mathbf{Y}=\left(\tau_{i}\right) \quad \text { for } \quad i=1,2,3,4
$$

A significant difference between the previous network and this one in terms of the inputs and outputs is the fact that though the number of outputs has only increased by one, the number of input parameters has increasea by four in order to accommodate the extra transmission line in the network. Also, the topology variables $e(j), j=$ $1,2,3,4$ now take on 9 possible values as opposed to 4 previously. As these variables are not continuous throughout the input space, greater care must be taken to ensure that the data used in training is representative of the entire input space.

\subsubsection{Constructing the Four Interconnect Neural Network Model}

The number of inputs, outputs, and hidden neurons chosen are shown in Table 6.5 
Table 6.5: Features of neural network model (four interconnects)

\begin{tabular}{|l|r|}
\hline Feature & Value \\
\hline Number of inputs $(m)$ & 18 \\
Number of Outputs $(n)$ & 4 \\
Number of neurons in hidden layer $(p)$ & 40 \\
Size of model (in floating point numbers) & 924 \\
Size of training set & 4500 \\
Size of test set & 4500 \\
Data Generation Technique & NILT \\
Training time (in hours) & 48 \\
iverage training error & 0.0356 \\
Average test error & 0.04 \\
\hline
\end{tabular}

Due to the nature of the topology variables, as well as the relatively high dimensionality of the problem in general, large training and test sets are used. NILT is employed to create this data. The training resulted in an accuracy of about $96 \%$ over the test set. The resulting error in delay simulation of 100 random circuit configurations from the test set are plotted in Fig. 6.6.

\subsection{Five Recipient Nodes Connected to a Source}

This example is the same as the previous two models, conly the interconnect network now contains 5 interconnects. The input space, which accounts for all five interconnects and loads has a relatively large dimension of 22 . The output vector has 5 elements in it. The neural network is larrar, and hence the training time as well as the size of the training set were significantly larger than seen in previous examples.

The features of this network are shown in Table 6.6.

The training set used is of size 6000 , and after 72 bours of training, the average test error reduced to 0.0628 . The large training time and the relatively large set 


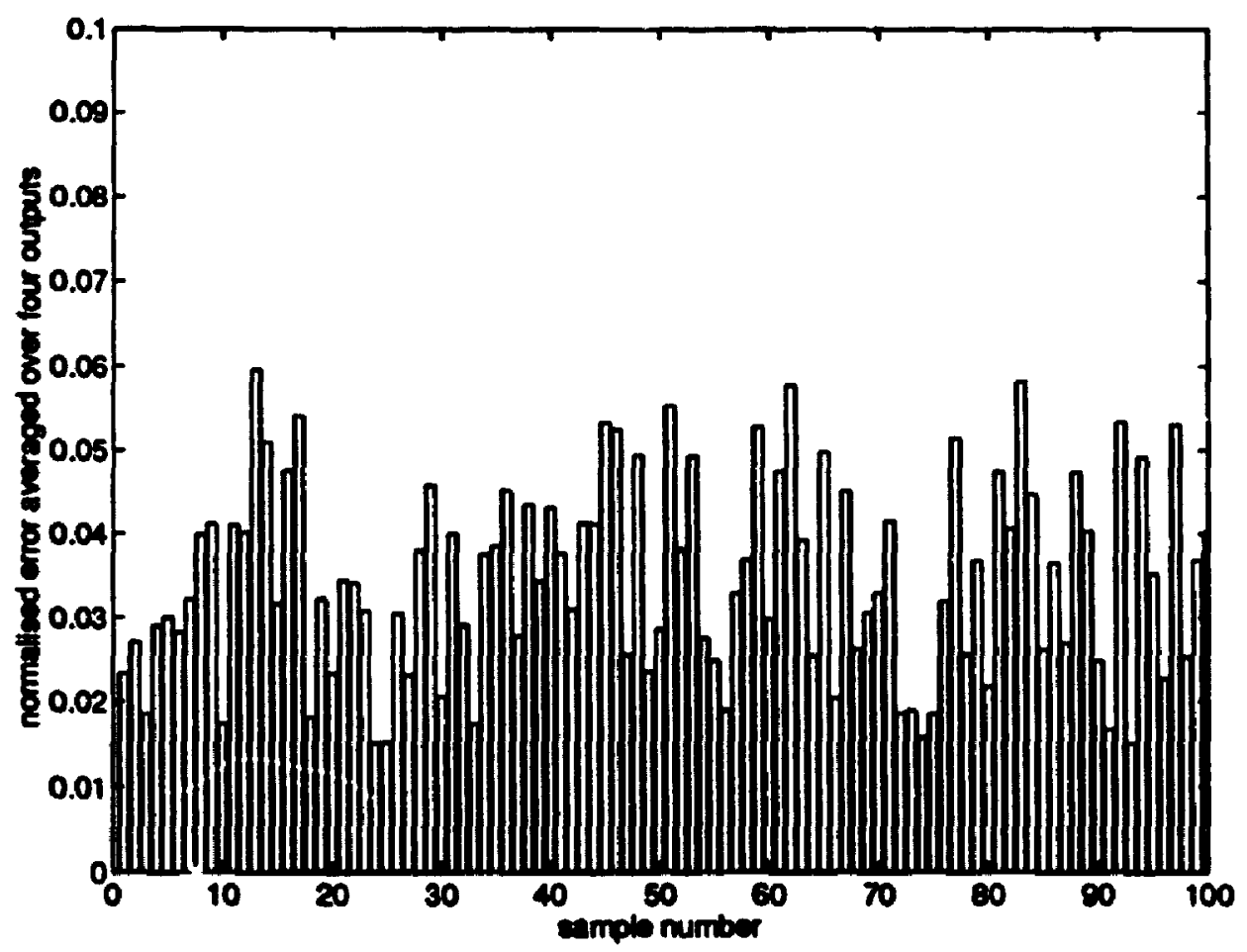

Figure 6.6: Error for 100 random circuit configurations for the four interconnect network 
Table 6.6: Features of neural network model for five interconnects

\begin{tabular}{|l|r|}
\hline Feature & Value \\
\hline Number of inputs $(m)$ & 22 \\
Number of Outputs $(n)$ & 5 \\
Number of neurons in hidden layer $(p)$ & 50 \\
Size of model (in floating point numbers) & 1405 \\
Size of training set & 6000 \\
Size of test set & 3000 \\
Data Generation Technique & NILT \\
Training time (in hours) & 72 \\
Average training error & 0.0593 \\
Average test error & 0.0628 \\
\hline
\end{tabular}

of training data were warranted by the size of the model. The error of the neural network model in simulating 100 random test vectors is shown in Fig. 6.7.

\subsection{Interconnect Networks of Other Sizes}

Networks of sizes three, four, and five have been modeled so far. This section considers interconnection networks of sizes other than those discussed.

\subsubsection{The Cases of A Single Interconnect and Two Intercon- nects}

A circuit in which there is a direct interconnection between a source and an receiver pin through one interconnect cannot have varying topology. Following the same input vector structure as the above examples, the input vector would have six elements, three describing the source, the interconnect length, and the termination resistance and capacitance. The output would be the delay at the receiver. In the absence of topology variation, and the reduced dimensionality of the input, modeling the single interconnect network structure would be much simpler than the previous examples. 


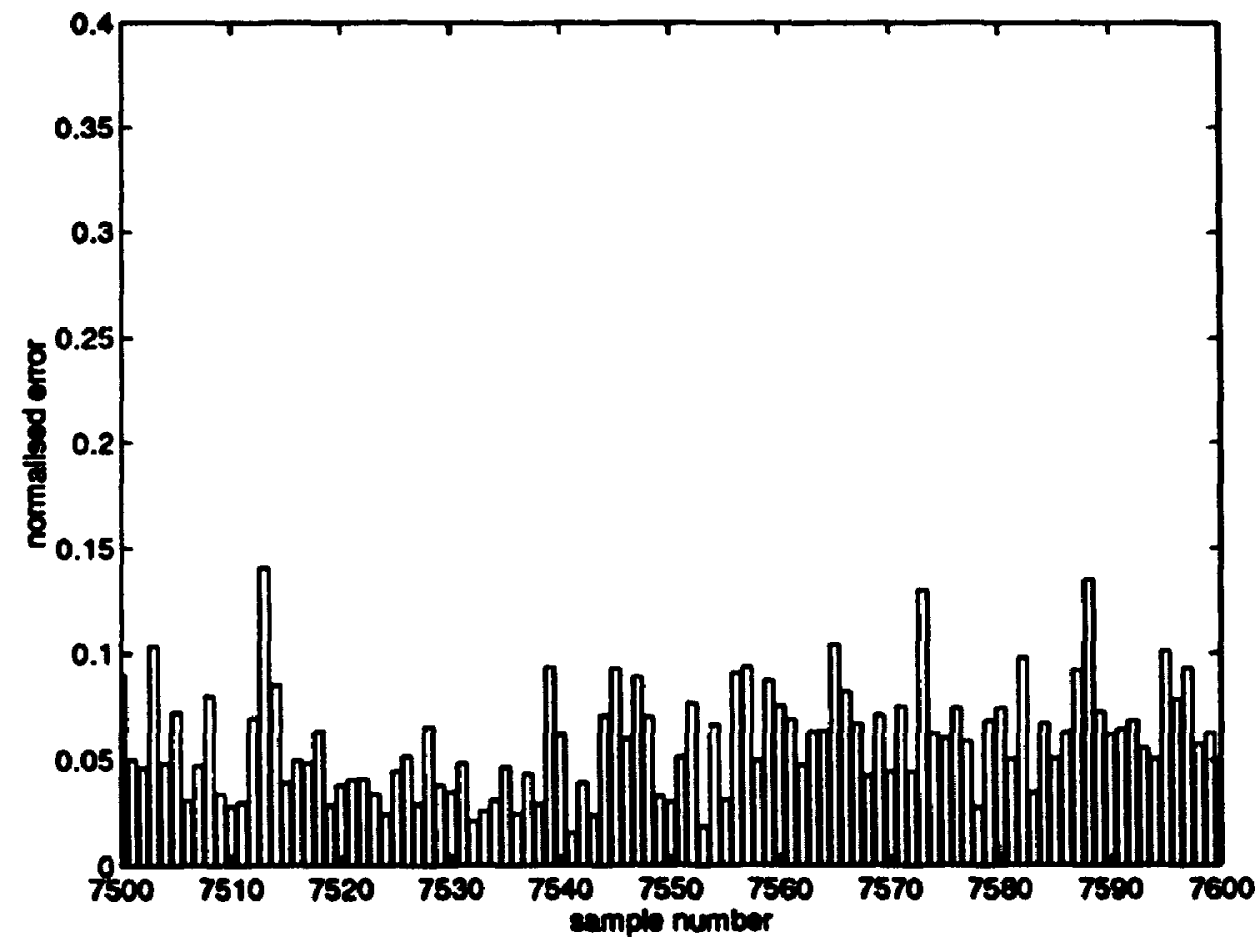

Figure 6.7: Error for 100 random circuit configurations for the five interconnect network 
Similarly, the two interconnect network would be relatively easy to model as it contains a single topology variable to allow for switching between its two possible configurations. These two cases are subsets of the larger models developed so far, and hence are not reported separately.

\subsubsection{Extra Large Networks}

Interconnect networks of size six or more can also occur in an electrical system. The size of the input vector for models of such networks would increase proportionately, to 26 for 6 interconnects, and 30 for the case of seven interconnects. Also, it can be seen from retabtop that the number of possible configurations the networks can assume increases to relatively larger values. The modeling of these networks is possible, but would require tremendous CPU resources, as it can be noted that the resources required for modeling increase as the number of interconnects increases. Investment of such resources is profitable only if the problem involved ensures very frequent use of the model, which, in the case of a typical digital PCB, is not the case. This is discussed further in Section 6.8.2

\subsection{Performance Comparison with Other Electri- cal Simulators}

In order to measure the performance of the neural network models, their CPU runtimes are compared with existing electrical simulation techniques, as has already been reported in [88].

The computational effectiveness of a model to be applied in an iterative optimization routine can be estimated by the run-time required for usage of the model a specific number of times. During each iteration of the optimization routine, the 
model is called once for every occurrence of the structure in the system. Also, the more iterations performed, the more reliable the results of optimization. A conservative estimate of the number of times a model needs to be called during optimization to obtain at least a reasonably (if not globally) optimal solution in an optimization problem is taken as 20,000 . The run-time requirements of the neural network model, NILT, and AWE are shown in Table 6.7 for 20,000 circuit simulations. The speed-up ratio is also shown.

Table 6.7: Run-time for simulation using the neural network model, NILT and AWE

\begin{tabular}{|l|l|l|}
\hline Method & CPU time required & Speed-up Ratio \\
\hline NILT & 34.43 hours & 310 \\
\hline AWE & 9.56 hours & 86 \\
\hline Neural Network Model & 6.67 minutes & 1 \\
\hline
\end{tabular}

\subsection{Discussion of Results}

The examples presented in this chapter allow further insight into the application of neural networks to electrical circuit simulation. This section highlights the main characteristics of the neural network approach to interconnect network analysis.

\subsubsection{Evaluation of the Simulator}

The most significant difference between the neural network models and traditional simulation techniques is the trivially small run-times associated with neural networks. The timing comparisons reveal a speed-up of roughly two orders of magnitude. To a circuit designer, this translates into a hundred times smaller time requirement for electrical layout optimization, or, given the same time frame, a hundred times more 
thorough an optimization search.

The input spaces of the models in this chapter were all of much higher dimensionality than the examples presented in Chapter 4. This is largely because the interconnects are simulated in an entire circuit environment, where the number of variables is much larger, and many more design variables must be accounted for. Even so, the neural networks could map the multi-dimensional input spaces to the outputs with relative ease, although the size of the networks, the training data set and the training time had to be proportionately increased. The final accuracy was still within reasonable tolerance levels.

Another noteworthy feature of the input spaces in this chapter were the topology variable set $e_{j}, j=1,2, . . n-1$. These variables are unconventional as they do not represent an absolute physical quantity which increases or decreases with their absolute value. They do not assume continuous values over the input space, and are not continuously differentiable. Although attempts were made to use a systematic numbering scheme which would give a semblance of a relationship with the output, the output space was, in general, not monotonic with respect to the values of $\boldsymbol{e}_{j}$. Even so, the neural networks were capable of accurately incorporating them into the input/output mapping function.

\subsubsection{The On-line/Off-line Time Trade-off}

An important issue that becomes apparent upon considering the training time and size of data sets used in this chapter is the off-line CPU time required to implement neural network models. The off-line time which must be invested in creating a neural network model can be expressed approximately as the sum of the training time, and the sum of the data generation time required for producing the training/test sets. In 
other words,

Off-line Time $=$ Data Generation Time + Training time

Since both the size of the data sets and the training time are direct functions of the input and output space, it can be inferred that problems of large dimensionality require a large off-line investment. Hence their use should be limited to cases where the on-line CPU time saved offsets the off-line investment, so that the original objective of CPU time reduction is achieved.

In the case of interconnect network simulation, the CPU off-line time necessary for creating neural network models for interconnect networks of size six or more might not be offset by their use in on-line PCB layout optimization routines, as these large occur relatively infrequently. Even in the three examples described in this chapter, the training time can be observed to have increased from 36 to 72 hours to maintain acceptable accuracy levels as the size of the interconnect networks increased.

If neural network models are employed for the majority of the simulations, while a traditional simulator is entrusted with the task of simulation of circuits or networks on the PCB which occur less often (and hence do not warrant the time investment of off-line modeling), a balance can be achieved between on-line time saving and off-line time investment, thereby achieving an overall reduction in simulation time. 


\subsection{Summary}

In this chapter the use of neural networks as an electric circuit simulation tool was studied. Neural network models that constituted a high-speed interconnect network simulator that can be used in propagation delay estimation were described, and the results analyzed. The quantification of network topology as an explicit design variable was described. The examples revealed the capacity of neural networks to map relationships of very high dimension which incorporated discontinuous variables and still offer substantial speed-up. The final section discussed the trade-off between on-line saving and off-line investment in terms of CPU time. 


\section{Chapter 7}

\section{Modeling the On-Chip Spiral Inductor}

\subsection{Introduction}

In this chapter, the application of neural network techniques to modeling the monolithic spiral inductor in a silicon-based production technology is studied. After establishing suitable modeling parameters and an input variable range, the model is constructed and compared with existing techniques. It is then demonstrated how the model can be used on-line as a powerful circuit design and analysis tool.

\subsection{The On-Chip Spiral Inductor Model}

The spiral inductor is an essential component of many analog circuits for RF applications. As discussed in Chapter II, accurate characterization of the inductor requires 3-D electromagnetic simulation. As this approach is impractical for use in on-line iterative CAD techniques, neural networks are applied to develop a model relating the physical/geometrical parameters with the design variables based on data obtained 
off-line.

A typical spiral inductor is shown in Fig. 7.1.

\subsubsection{Definition of Input/Output Parameters}

The input parameters to the neural network model are the physical parameters as shown in Fig. 7.1, namely the outer length, the conductor width and separation, the number of turns, and the frequency of operation. A model is developed for the square spiral inductor, but this can be extended to any type of spiral (e.g. rectangular, circular, or octagonal). In many design situations, the number of turns is not an integer, and hence this parameter can assume fractional values as well. The outputs modeled are the individual elements of the two-port $S$-parameters matrix defining the structure. Since $S_{12}$ is equal to $S_{21}$, they need to be considered only once. Thus the input layer vector, $\mathbf{X}$, is given by

$$
\mathbf{X}=(N, L, W, s, \omega)
$$

and the output vector from the neural network, $Y$, is

$$
Y=\left(\operatorname{Re}\left(S_{11}\right), \operatorname{Im}\left(S_{11}\right), \operatorname{Re}\left(S_{21}\right), \operatorname{Im}\left(S_{21}\right), \operatorname{Re}\left(S_{22}\right), \operatorname{Im}\left(S_{22}\right)\right)
$$

The ranges of the input parameters are shown in Table 7.1, and are nominal ranges for the application and technology employed, which are for a front-end receiver for PCS.

\subsubsection{Construction of the Model}

The technique used to generate data in the case of the monolithic inductor model is as described in [50]. The electromagnetic behaviour of the monolithic inductor from the 


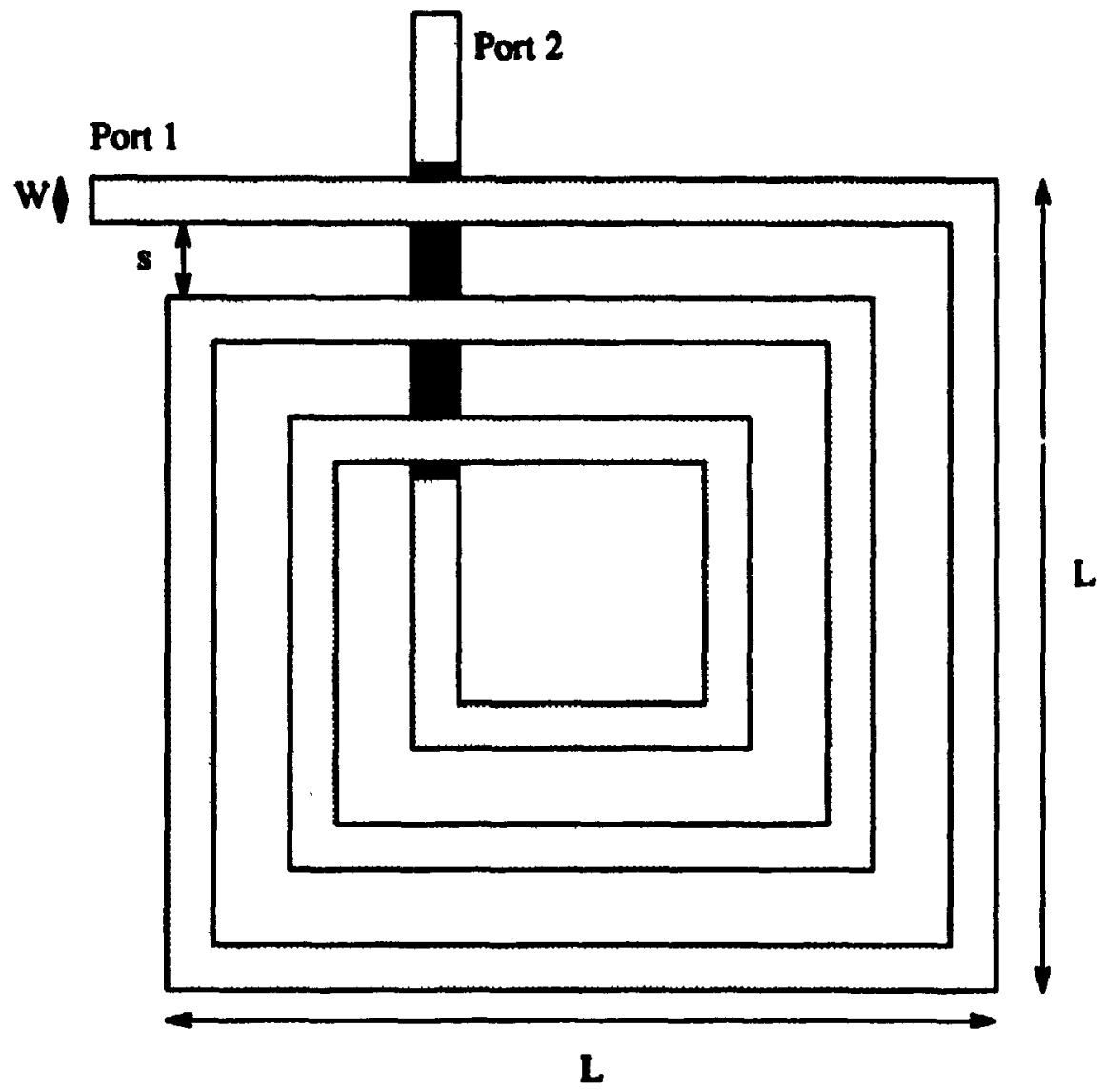

Figure 7.1: The square spiral inductor 
Table 7.1: Input parameters for the inductor model and their range

\begin{tabular}{|l|l|r|r|}
\hline Parameter & Symbol & Minimum & Maximum \\
\hline Conductor Width & $W$ & $4 \mu \mathrm{m}$ & $20 \mu \mathrm{m}$ \\
Separation & $s$ & $1.5 \mu \mathrm{m}$ & $7.5 \mu \mathrm{m}$ \\
Outer Length & $L$ & $19 \mu \mathrm{m}$ & $675 \mu \mathrm{m}$ \\
Number of Turns & $N$ & 1 & 9 \\
Frequency & $\omega$ & $1 \mathrm{MHz}$ & $10 \mathrm{GHz}$ \\
\hline
\end{tabular}

circuit layout and substrate parameters is described using a physically-based lumpedelement model. The values of the lossless lumped elements are obtained from the physical parameters through quasi-static EM simulation, while the lossy parameters are characterized by closed-form relationships.

The characteristics of the neural network are as listed in Table 7.2.

Table 7.2: Features of the neural network model

\begin{tabular}{|l|r|}
\hline Feature & Value \\
\hline Number of inputs $(m)$ & 5 \\
Number of outputs to overall model & 8 \\
Number of outputs to neural net $(n)$ & 6 \\
Number of neurons in hidden layer $(p)$ & 25 \\
Size of model (in floating point numbers) & 306 \\
Size of training set & 500 \\
Size of test set & 500 \\
Data Generation Technique & Quasi-TEM Simulation \\
Training time (in hours) & 40 \\
Average training error & 0.038 \\
Average test error & 0.04 \\
\hline
\end{tabular}

With 25 hidden neurons, the neural network was able to model the $S$-parameters of the inductor with reasonable accuracy. 


\subsubsection{Performance Comparison with Existing Techniques}

The accuracy of simulation of the $S$-parameters was comparable to the EM Field solver used. Fig. 7.2 shows the test error of 100 random structures of the neural network in comparison to the Field solver.

A comparison of run-time of the neural network model and other existing techniques EM is shown in Table 7.3, and it indicates the speed with which the $S$ parameters would be available to the designer.

Table 7.3: Comparison of on-line simulation run-time

\begin{tabular}{|l|l|r|}
\hline Method & Mathematical Operations On-line & Average run-time \\
\hline 3-D EM analysis & solution of structure in 3-D & $10-30$ minutes \\
\hline Quasi-TEM Simulation & $\begin{array}{l}\text { Quasi-TEM approximation and solution } \\
\text { of differential/integral equations }\end{array}$ & $2-5$ minutes \\
\hline Neural Network Model & $\begin{array}{l}\text { sum-of-products, } \\
\text { exponentiation }\end{array}$ & .003 seconds \\
\hline
\end{tabular}

In addition, the memory requirements of the neural network model are much lower when compared to the storage required by look-up tables for the inductor. While a neural network requires 306 floating point numbers to store information regarding the input/output mapping, a table look-up model, such as [54] requires over 900,000 floating point numbers to store data. Also, [54] does not allow for interpolation, and hence continuous derivatives are not be available. 


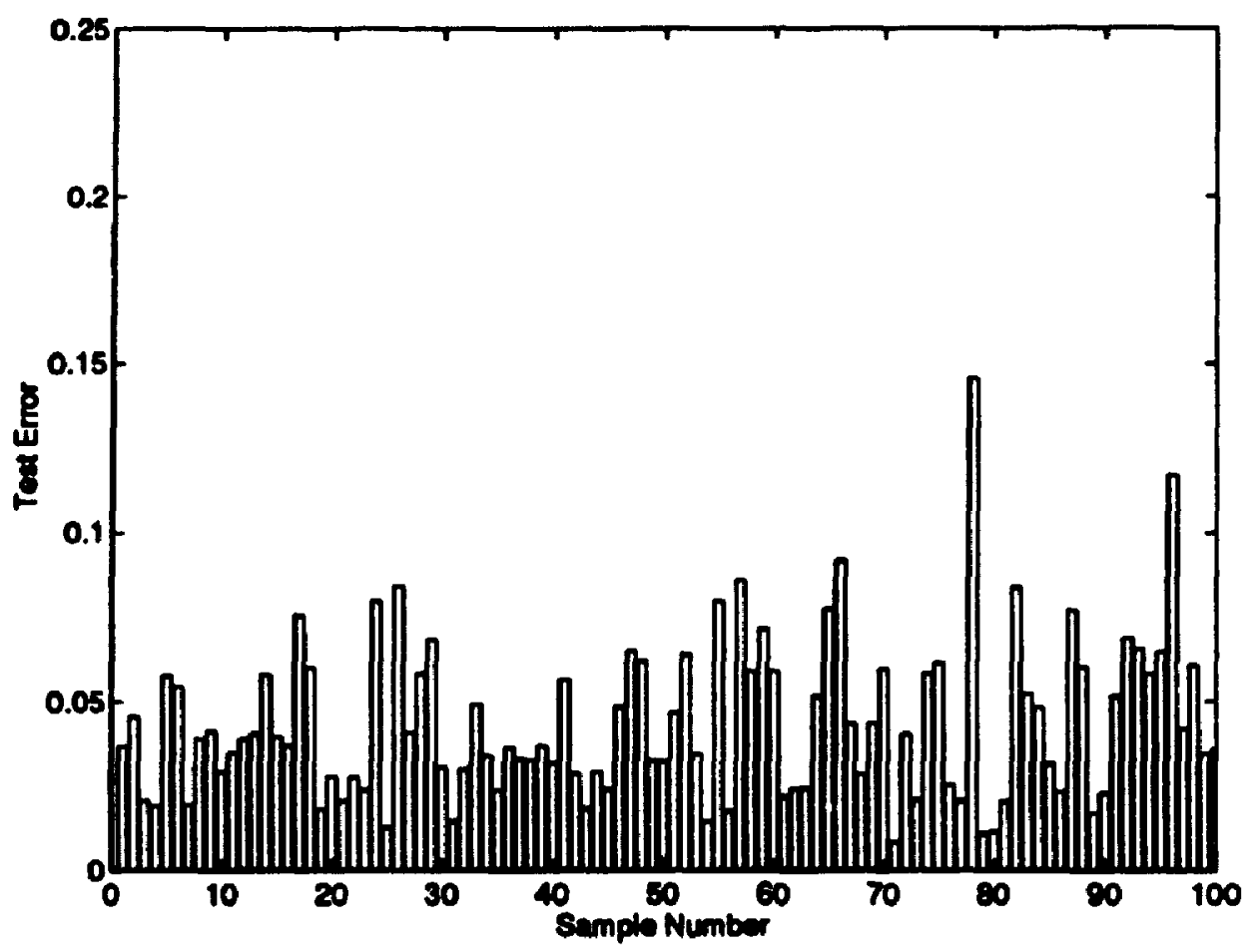

Figure 7.2: Test error in S-parameter simulation for 100 random spiral inductor structures 


\subsection{Applications of the Model}

\subsubsection{On-line Simulation of S-parameters}

The trivial run-time requirements of the neural network model imply that a designer is at liberty to check or simulate as many configurations as desired, and that CPU time is no longer a limiting factor in design optimization. This would open avenues for more elaborate computer-aided optimization techniques such as gradient-based optimization, simulated annealing, etc., which are based on iterative techniques and exhaustive variable-space searches.

Examples of the parameters $S_{11}$ and $S_{12}$ as simulated by the neural network for an 8-turn spiral inductor are shown in Fig. 7.3. The $S$-parameters are related to other design parameters such as the $Z$ and $Y$ parameters through algebraic transformations. Hence, once the $S$-parameters are known, they can be used to obtain performance and design characteristics such as Q-fuctor, inductance and other useful design information, through simple algebraic manipulation [96].

\subsubsection{Inductor Self-Resonant Frequency Analysis}

The self-resonant frequency of a spiral inductor is the frequency at which the inductor ceases to have positive reactance, but becomes capacitive in nature. Therefore, it indicates the useful frequency range or bandwidth of the inductor, and it is customarily specified along with the low-frequency inductance and the inductor Q-factor. The self-resonant frequency can be determined from the reactance characteristics of an inductor as the frequency at which the reactance becomes negative. The inductance curves are plotted in Fig. 7.4 for spirals varying from four to nine turns, with all other parameters of the spiral kept constant. The self-resonant frequency, represented as 


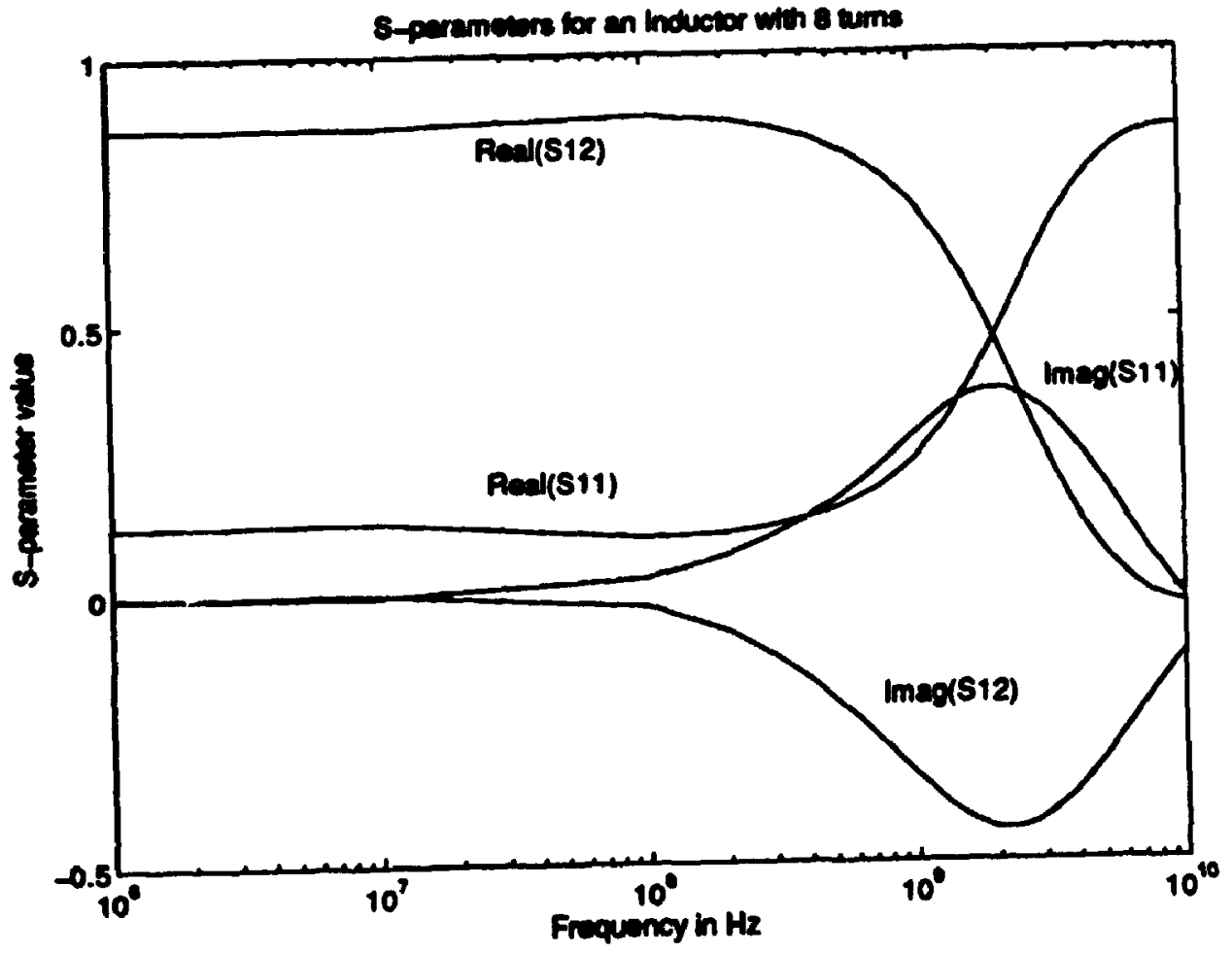

Figure 7.3: S-Parameters for a spiral inductor having 8 turns 
the point at which the inductance becomes negative in Fig. 7.4 , is seen to decrease as the number of turn increases. This is consistent with the well known behaviour of spiral inductors fabricated in other IC technologies [96].

An exhaustive parameter sweep was performed using the neural network model to isolate inductors of different physical parameters which had the same inductance. Their self-resonant frequencies were then studied. A total of thirty-two inductors, all having an inductance of $15 \mathrm{nH}$ were found from the parameter sweep. These inductors were then analyzed for their self-resonant frequency, which involved determining the frequency at which their overall inductance is zero. The physical dimensions and self-resonant frequencies of four of the structures are listed below in Table 7.4.

Table 7.4: Geometries and self-resonant frequencies of inductors having a lowfrequency inductance of $15 \mathrm{nH}$

\begin{tabular}{|c|c|c|c|c|}
\hline Number & $\mathrm{N}$ & W $(\mu \mathrm{m})$ & $\mathrm{L}(\mu \mathrm{m})$ & Self-resonant Frequency $(\mathrm{GHz})$ \\
\hline 1 & 6 & 16 & 354 & 4.3 \\
2 & 7.5 & 17 & 347 & 4.6 \\
3 & 8.5 & 10 & 267 & 5.2 \\
4 & 9 & 20 & 427 & 3.0 \\
\hline
\end{tabular}

No consistent relationship is apparent from inspection of the above data with regards to how the self-resonant frequency varies with the number of turns or the conductor width. However, it should be noted that the self-resonant frequency is related to the capacitive parasitics of the inductor, which are defined by the surface area of the spiral. Thus, the self-resonant frequency is seen to decrease consistently as the metallized area of the inductor increases.

This relationship is seen more clearly from the three-dimensional plot shown in Fig. 7.5. The parameter sweep was repeated a second time keeping the number of 


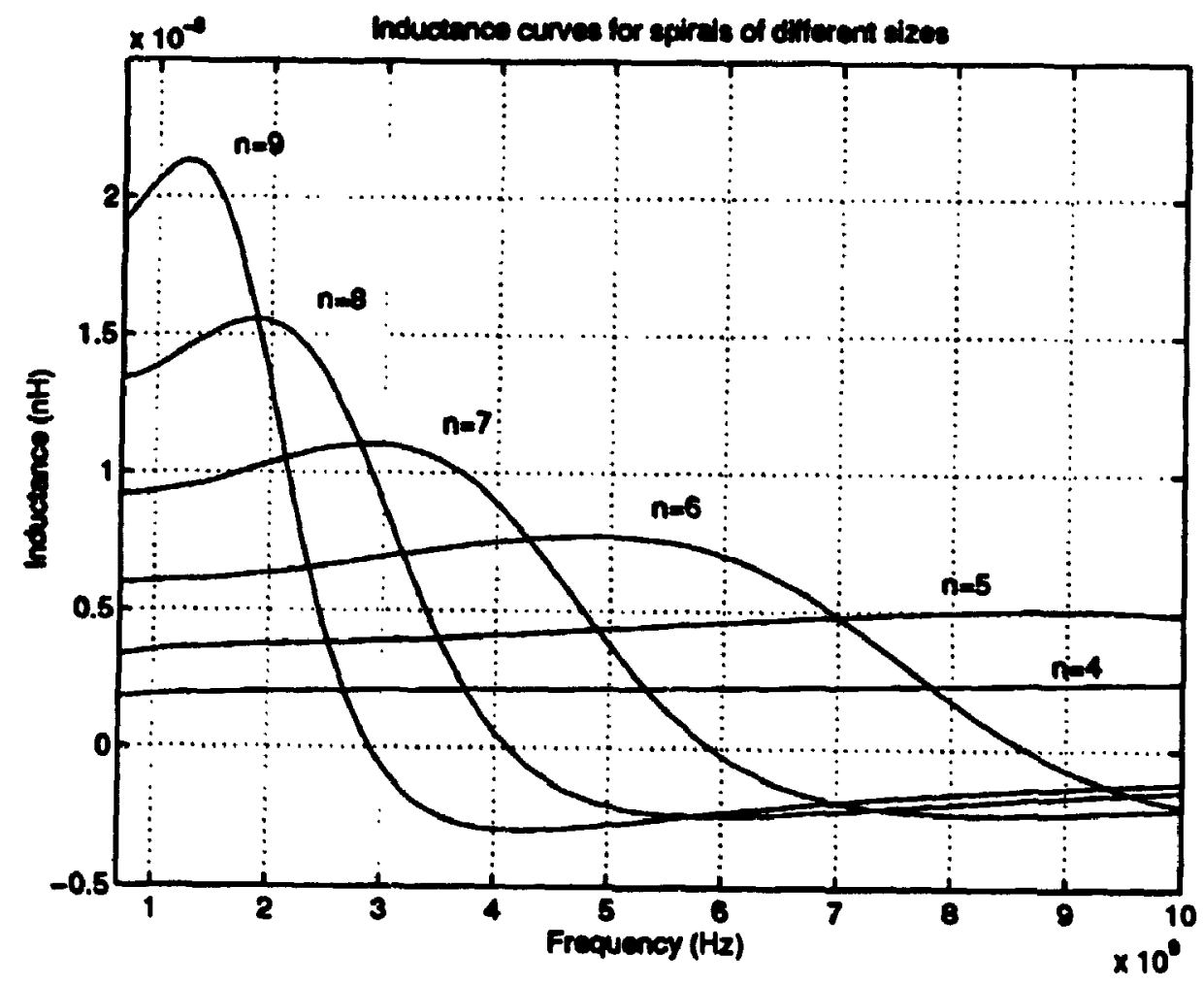

Figure 7.4: Inductance as a function of frequency for spirals of different sizes 
turns of the structure fixed at nine. For a low-frequency inductance of $15 \mathrm{nH}$, nine spirals of different dimensions were found. For an increase in the conductor width and outside dimensions of the inductor, both of which tend to increase the surface area of the spiral, there is a consistent lowering of the self-resonant frequency. From this it can be concluded that for a given inductance, the self-resonant frequency is maximized through optimization of the metal area which is used to define the inductor spiral.

Such information can only be obtained by performing exhaustive parameter sweeps, which would require very large computational resources if performed using traditional techniques.

\subsubsection{Q-Factor Analysis}

The fast computation allowed by the neural network model of the spiral inductor opens up new possibilities for component analysis and visua - zation of design data. Here the neural network is applied to the analysis of the inductor Q-factor as a demonstration of this capability.

The spiral inductor's quality factor can be expressed as

$$
\text { Q-factor }=\frac{w L}{R}
$$

A high value of $Q$-factor is needed for the best performance in filtering applications using spiral inductors.

A larger value of inductance might be expected to give a higher inductor $Q$-factor. However, the parasitic series resistance of the metal winding increases along with the inductance, and this tends to reduce the $Q$. As discussed in the previous section, large inductors consume more die area and therefore have a lower self-resonant frequency. 


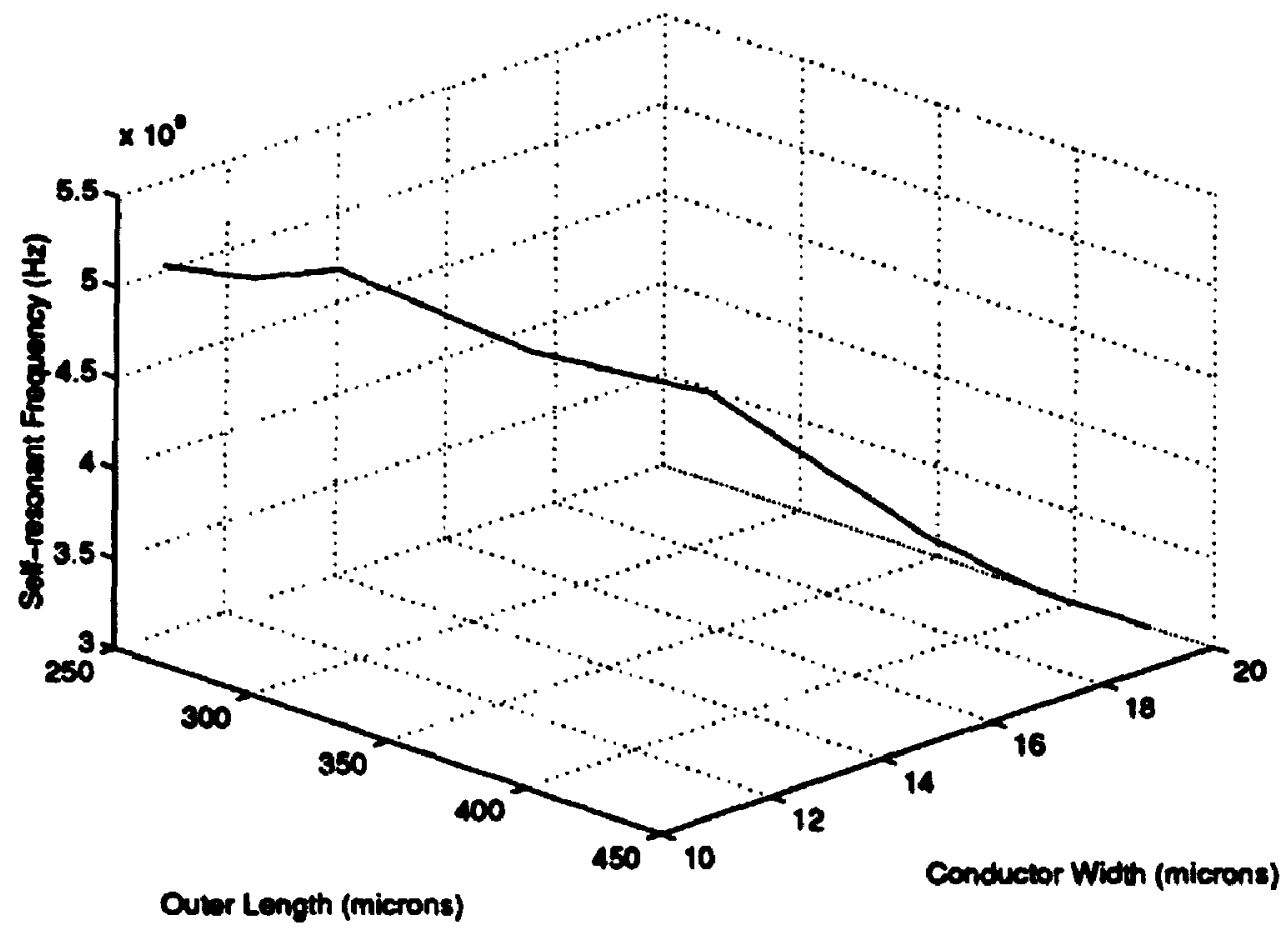

Figure 7.5: Self-resonant frequency versus physical dimensions for a fixed number of turns 
Thus, the Q-factor is also influenced by the inductor area, and a careful consideration of the number of turns, conductor width and spiral dimensions is required to optimize the inductor performance with respect to both Q-factor and self-resonant frequency.

The neural network moael of the spiral inductor is well-suited to an in-depth analysis of the relationships between design parameters. In Fig. 7.6- 7.8, the Q-factor of an inductor is plotted while varying the number of turns and the conductor width, the length varying proportionately with the other dimensions. The Q-factor surface is seen to evolve considerably as the frequency changes, with the peak $\mathbf{Q}$ obtainable shifting to a lower number of turns and a narrower conductor.

The graphical representation of the Q-factor is a valuable tool to the designer, which can be exploited to investigate the design space. It allows an IC designer the freedom to examine the relationships between the inductor parameters and the inductor performance. The relationships between parameters and performance can be visualized in a way that yields productive new insights into the optimization of spiral inductors in silicon technology. 


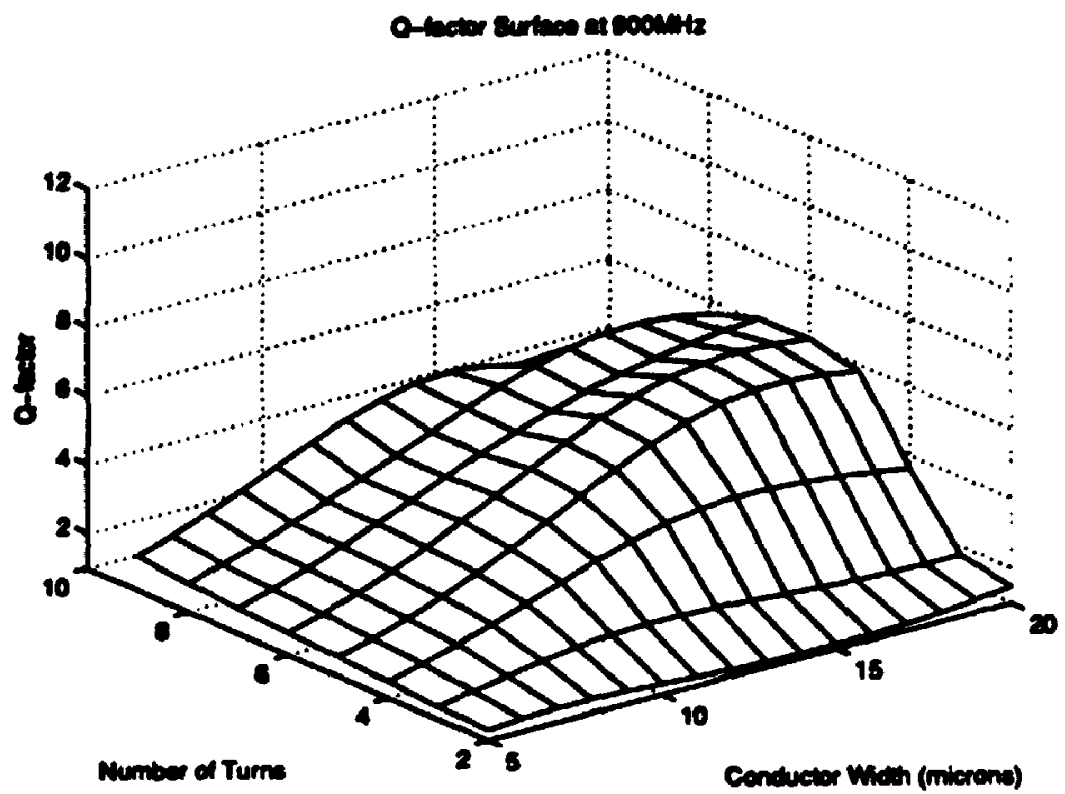

Figure 7.6: Q-factor surface at $900 \mathrm{MHz}$

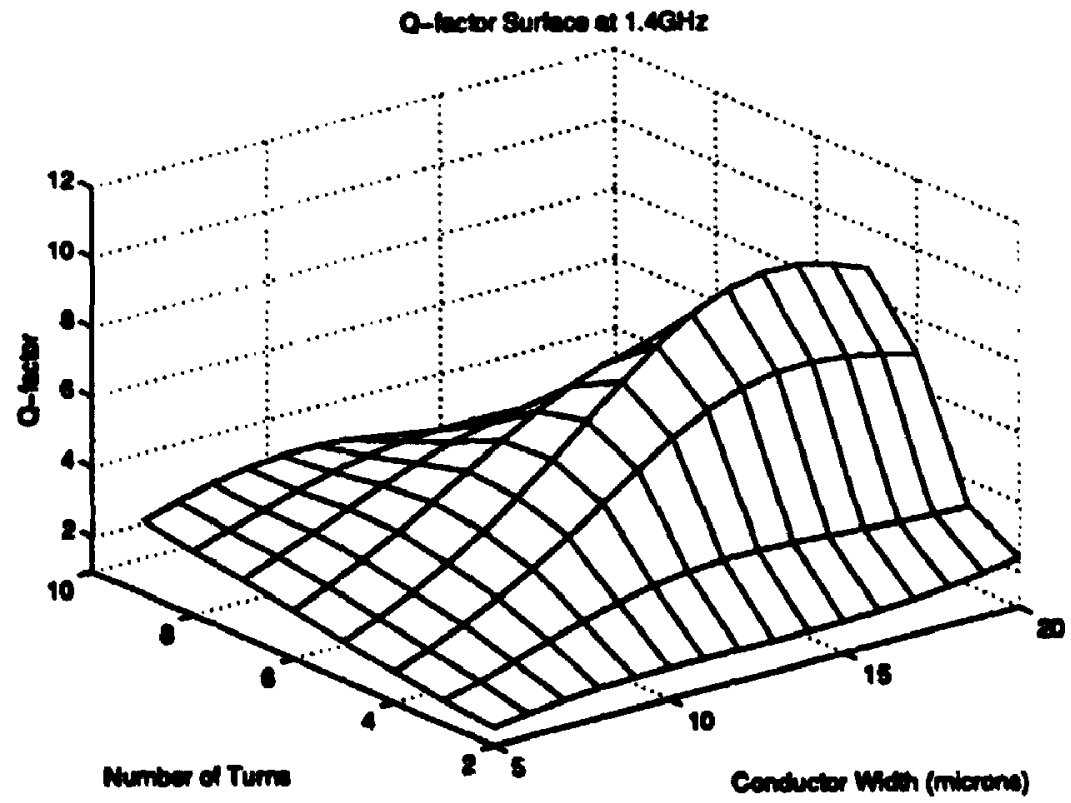

Figure 7.7: Q-factor surface at $1.4 \mathrm{GHz}$ 


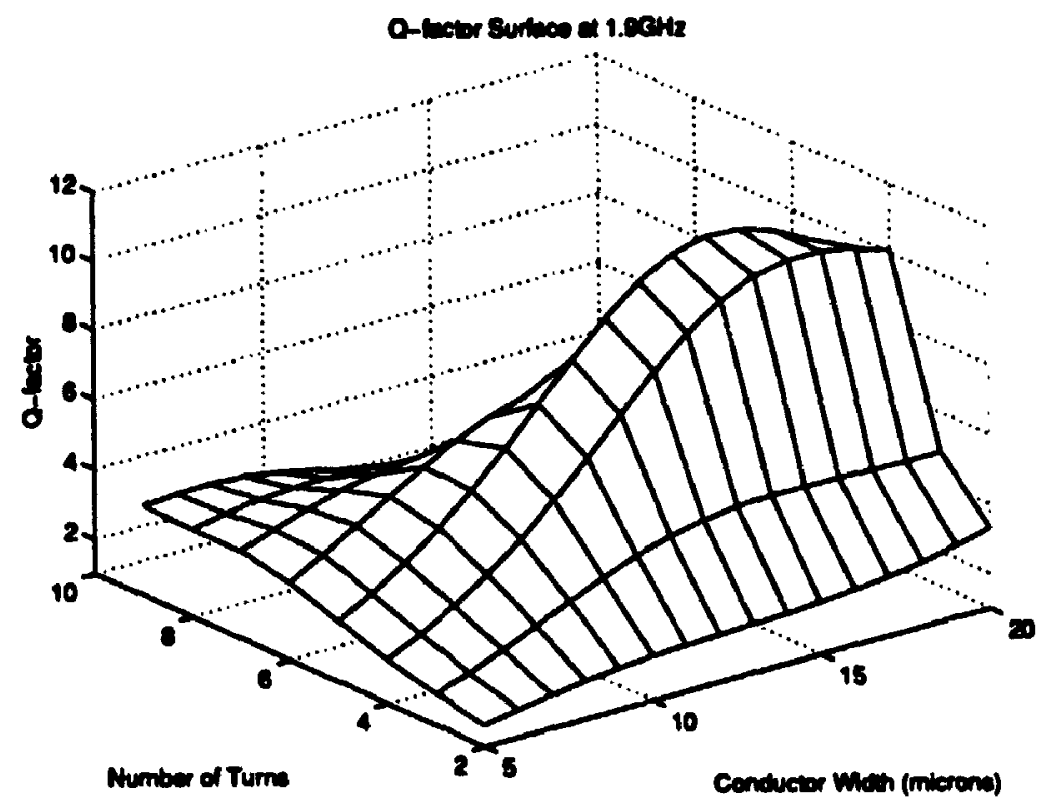

Figure 7.8: Q-factor surface at $1.9 \mathrm{GHz}$ 


\subsection{Summary}

In this section, neural network techniques were successfully extended to modeling the monolithic inductor. The on-line speed offered is very useful in studying the behaviour of the inductor and how altering the physical and geometric properties of the structure changes the design parameters. Upon comparison of the performance of the model with that of existing simulation techniques, it was found to be substantially faster and more compact in terms of memory requirements. 


\section{Chapter 8}

\section{Summary and Conclusions}

\subsection{Summary}

This thesis has aitempted to present an alternative approach to developing fast on-line models for passive structures for VLSI and RF circuit design, based on neural networks.

A review of recent literature and trends in CAD techniques for interconnects and passive microwave structures reveals that the complexity of the algorithms employed necessitate large CPU resource expenditure in analysis and accurate characterization of the structure. This is largely because they are based on numerical techniques which solve first-principle electromagnetic equations.

There is a large number of components in a typical high-speed system, and most currently employed CAD and optimization techniques are highly iterative in nature. Thus there is a an acute need for fast simulation techniques which require minimal on-line CPU resources so that CAD and optimization routines can converge in a minimal time frame. This need has spawned a prolific amount of research in modeling structures on the basis of data obtained either by off-line simulation or experimental 
measurement, in the forms of empirical relationships, table look-up techniques or simple lumped-element equivalents.

Neural networks have emerged as a very important problem-solving tool in several disciplines of the physical sciences and engineering. The rapid growth in computing technology in recent years has made neural network implementation more feasible, and several neural network architectures have been realized. The essential idea of learning on the basis of previous experience is common to all types of neural networks, and this principle makes neural networks a popular choice for problems constrained by the lack of fast or efficient algorithms. The three-layered backpropagation network can be applied to developing models for VLSI/RF circuit design, an area which is characterized by this problem. The feed-forward architecture is easily implemented, inherently stable and trained using the backpropagation algorithm.

The neural network is capable of modeling coupled transmission lines for their frequency-dependent parameters RLCG parameters. On-line speed-up, and the capacity to handle a wide range of frequencies, are among the advantages of using a neural network approach to this problem.

Networks of interconnects in high-speed VLSI circuits can be simulated for signal integrity parameters such as propagation delay using the neural network approach. This necessitates the expression of network topology as an explicit variable, as layout problems involve the varying of topology. A speed-up in the range of two orders of magnitude can be achieved on-line measured against traditional EM field simulation techniques.

The application of neural networks to modeling the monolithic inductor in RF circuit design facilitates calculation of the S-parameters and other associated design parameters from its physical dimensions. Thus neural network based models can be 
used as a powerful design tool capable of optimization and design based on extensive parameter-sweep routines.

The following section evaluates the neural network approach to CAD at high frequencies.

\subsection{Neural Network Models in Perspective}

\subsubsection{Advantages}

There were several advantages seen in the neural network approach in the applications discussed in the previous three chapters. They are summarized below.

Computational Speed-up The on-line speed-up achieved by the neural network models in all the examples were in the range of two to three orders of magnitude. This implies that a circuit designer need not be too concerned with the CPU resources expended. Restrictions imposed on the number of signal paths chosen as being critical to design in the case of interconnects, or on the number of iterations allowable in optimization due to time budgets constraints can now be eased, resulting in improved design.

Low Memory Requirements The examples discussed in Chapter 5 show that even relatively large models with a large number of outputs do not have huge memory requirements. Similarly, in Chapter 7, the inductor model required much less memory than a look-up table. Interpolation was not allowed in this case either.

Capacity to Handle A Wide Range of Frequency Neural network models operate over the entire spectrum of frequency, or any other variable for that matter, so long as training data is available. This is not the case in several current 
techniques, which require different approaches at different frequencies.

Capacity to Handle Network Topology The capability of neural networks to handle relatively unconventional variables such as network topology is an indication of the freedom with which applications for neural network models can be construed. Confinement to traditional variables representing absolute quantities is not necessary in a neural network approach.

Availability of Derivatives The input/output relationship in a neural network as expressed by Equations 4.4-4.7 are differentiable and hence the first order derivatives can be obtained. Thus sensitivity analysis would be possible.

\subsubsection{Disadvantages}

The applications in the previous chapter have also revealed several disadvantages inherent in the neural network approach.

Large Training Resources Long training times and large amounts of data are required to produce an accurate model. If insufficient data is provided, a neural network cannot be trained to a high degree of accuracy.

Difficulty in deciding Neural Network Parameters No fixed algorithms exist to determine the correct neural network structure to be used (the number of hidden layers and neurons, etc.), and the optimum training parameters (learning rate, etc.).

No Relationship to Physical Nature of Problem The weights and biases in a neural network do not bear any relationship to the physical nature of the problem. Usually, in characterization using closed-form equations, the coefficients 
of the formulas have physical significance, or represent some aspect of the phenomenon being modeled. This is unfortunately not true for neural networks, which is a true black-box technique.

\subsection{Performance Evaluation Against Existing Tech- niques}

In this section, the neural network approach is evaluated individually against the modeling techniques presently used in a typical CAD environment, as outlined in Fig. 2.1. The advantages and disadvantages of the neural network technique, as well as those of existing techniques in the context of neural networks are examined.

\subsubsection{Electromagnetic-based Simulation}

- In comparison to electromagnetic simulation techniques, the low run-time requirements are the most significant advantage of using neural networks. The run-time comparisons made in all of the applications, as reported in Tables $5.6,6.7$, and 7.3 indicate this.

- Run-time memory requirements are also significantly lower when neural networks are used. Electromagnetic techniques which use methods such as FEM must allocate large amounts of memory during execution. For instance, a large nine-turn inductor having wide spacing and narrow conductor widths would require substantial dynamic memory resources to be simulated. When simulating such structures, EM Field solvers cannot run on machines which share resources with other programs. 
- Neural networks can make use of measured data to develop models. For complex structures and structures in which several of the variables are either unknown, or unquantifiable using classical techniques, it is necessary to rely on experimentally measured data for modeling. This is especially true for structures such as the monolithic inductor, where measured data is often relied upon for characterization.

However, electromagnetic simulation techniques which use netiists as a basis of input have the advantage that the entire input space need not be known beforehand. Variables such as the network topology, or the number of metallic conductors running in parallel, which had to be carefully considered during neural network modeling, are implicitly defined in netlists, and hence are handled in an easier manner.

\subsubsection{Table Look-up Models}

- In comparison to look-up tables, the memory requirements of neural networks are significantly lower, as was $s^{\prime}$, own in Section 7.2.3. Also, the size of a neural network does not grow exponentially with the number of input variables, as a look-up table would.

- A significantly lower number of data points (from off-line simulation or measurement) are required to construct a neural network model, in comparison to a table, which requires a data point for every entry.

- Interpolation is possible with neural networks, and the derivatives at any point within :he in put space are available. Look-up tables often do not provide interpolation or derivatives at points between actual entries. 
However, look-up tables offer two significant advantages over the neural network approach,

- Any degree of non-linearity between the input and output can be handled by a neural network, as there is no sense of a relationship between the data stored (the outputs) in the table and their addressees (the input parameters). The neural network approach is confined to problems where a relationship exists to be mapped.

- Look-up tables are generally simpler to create and update than neural networks, as they do not require training.

\subsubsection{Closed-Form Equations and Curve-Fit Techniques}

There are several advantages of using neural networks over polynomial curve-fit techniques or closed-form equations. These include

- Neural networks are capable of handling a much larger number of input variables, as was demonstrated ir. Chapter 6.

- The number of output variables that a neural network can simultaneously handle is significantly higher thin that of curve-fit techniques. For example, in the eight-bit digital data bus example in Chapter 5, all thirty outputs were simultaneously handled, while if curve-fit techniques were employed, each of these would have to be done separately.

- The correct spline, and values of the coefficients of the polynomial equation must be determined by the designer. In the neural network approach, this 
process is replace by training, which is simpler and requires less effort from the designer.

Closed-form equations have a slight edge over neural networks due to the fact that they are closely related to the physical nature of the problem, and thus provide better understanding of the problem. As they are based on simplification of classical techniques and equations, the relationship between the input and output is apparent to the user, unlike the neural network which is essentially a black-box technique.

\subsubsection{Lumped-Element Equivalents}

Lumped-element models of complex high frequency structures have the advantage that they can be easily incorporated into existing simulators, and hence are a popular front-end simulation technique. However, the difficulties involved in using lumpedelement techniques are

- Lumped-element equivalents of complex structures are normally highly restrictive on the valid range of frequencies at which they can be used. Thus different circuits must be developed for different ranges of frequency.

- It is often difficult to decide the correct circuit configuration to optimally model a structure, and to assign optimum values to the different elements. This is analogous to the difficulties that arise in determining the correct degree and values of coefficients of a polynomial in curve-fit techniques.

Many optimizers and simulators used nowadays allow for incorporation of external subroutines and programs for models. Hence it is as easy to use neural network-based models as it is to use lumped-element techniques. 


\subsection{Applying the Neural Network Technique}

As was discussed in Chapter 6, an important consideration while developing neural network models for large problems is the off-line investment called for in terms of CPU time. According to Equation 6.5, the larger the problem in terms of complexity and dimensionality, the greater the investment. This is, in general, true of numerical techniques, that the greater the flexibility of the problem in terms of design variables, or the greater the complexity of the problem, the more effort is required. A few general guidelines are offered below to give a better understanding of where neural networks would be best suited.

1. Multi-dimensional problems where the inputs are known to be related the the outputs through smooth continuous curves can be modeled using neural networks.

2. Neural networks can be applied to problems for which a memory look-up table is currently employed, where a mildly non-linear relationship exists between the input and output. In such cases, no time is expended in generation of data, as the data in the look-up table can be used for training and testing the neural network model.

3. Complex relationships for which analytical expressions or numerical algorithms do not exist, where field measurement data is largely relied upon for design can be modeled using neural networks.

4. Neural networks can be employed in modeling relationships which one would traditionally attempt to curve-fit or find closed-form equations. The idea of 
interpolating measured data is common to both approaches, but determining the relationship is much easier with neural networks.

5. Frequently used models, even if they are extremely complex and high in dimension, can be modeled using neural networks, as the the off-line CPU time would be justified.

\subsection{Suggestions for Further Research}

The technique of neural network modeling can be extended to several other CAD applications.

1. Modeling of cells and larger blocks of components used in semi-custom layout can be done using neural networks.

2. The modeling of other microwave components, such as overlay capacitors, is generally highly CPU intensive, and would be a good application for neural networks.

3. The monolithic inductor model can be expanded to be able to handle different production technologies, such as Gallium Arsenide. This can be done by adding substrate resistivity as a variable to the input vector. This variable would assume a very high value in the case of Gallium Arsenide.

Furthermore, the network simulation problem of Chapter 6 can be extended to high dimensions such as eight or nine interconnects, but with a limit on the number of topologies allowed. For example, the output of a clock might be fed serially to nine elements, which would constitute a nine interconnect network, but only in series. If 
127

only the series case is modeled, there would be a significant reduction in the size of the input space and the amount of training/test data required for the problem.

A study of the relationship between the error in the output of the neural network, i.e. $\varepsilon$, and the input vector $x_{k}$ ) could indicate areas which would give higher error than others in a given neural network. This would greatly help in defining a level of confidence in the output of the neural network for a given input.

The application of recent advances in training and neural network configuration such as pruning, sparse techniques, etc. can be applied to the general method deviloped in Chapter 4, to help produce faster and more efficiently trained models. 


\section{Bibliography}

[1] R. Griffith, "Mixed Frequency/Time Domain Analysis of High Speed Interconnects", Master of Engineeri $g$ Thesis, Carleton University, Ottawa, July 1993.

[2] M.S. Nakhla and Q.J. Zhang, Editors, Mod ling and Simulation of High Speed VSLI Interconnects, Boston: Kluwer, 1994.

[3] R. Sainati and T. Moravec, "Estimating High Speed Circuit Interconnect Performance", IEEE Trans. Circuits Syst., vol. 36, no. 4, pp. 533-541, April 1989.

[4] D.S. Gao, A.T. Yang and S.M. Kang, "Modeling and Simulation of Interconnection Delays and Crosstalk in High-Speed Integrated Circuits", IEEE Trans. Circuits Syst., vol. 37, no. 1, pp. 1-9, January 1990.

[5] W.W.M. Dai (Guest Editor), "Special Issue on Simulation, Modeling and Electrical Design of High-speed and High-density Interconnects", IEEE Trans. Circuits Syst., vol. 39, no. 11, pp. 857-982, November 1992.

[6] J. Williamson, K. Kalaichelvan, M.S. Nakhla, Q.J. Zhang and P. Van der Puije, "Ground Noise Estimation and Minimization in Integrated Circuit Packages", Proc. IEEE Intl. Symp. EMC, pp. 425-428, Dallas, USA, August 1993.

[7] J. Vlach and K. Singhal, Computer Methods for Circuit Analysis and Design, New York: Van Nostrand Reinhold, 1983. 
[8] J.W. Bandler and S.H. Chen, "Circuit Optimization: The State of the Art", IEEE Trans. Microwave Theory \& Tech., vol. 36, no. 2, pp. 424-443, February 1988.

[9] K.K. Mihan, B.J. Stacey, M.S. Nakhla and Q.J. Zhang, "Concurrent Thermal and Electrical Optimization of High-Speed Packages and Systems", International Intersociety Electronic Packaging Conference, pp. 221-227, Kaanapali Beach, USA, March 1995.

[10] M.P. Veechi and S. Kirkpatrick, "Global Wiring by Simulated Annealing", IEEE Trans. Computer-Aided Design, vol. 2, no. 4, pp. 215-222, October 1983.

[11] S. Nahar, S. Sahni and E. Shragowitz, "Simulated Annealing and Combinatorial Optimization", Proc. IEEE Design Automation Conference, pp. 293-299, Las Vegas, USA, June 1986.

[12] OSA90 Version 2.0 Reference Manual, Optimization Systems Associates Inc., Dundas, Ontario.

[13] J. Rubinstein, P. Penfield and M.A. Horowitz, "Signal Delay in RC Tree Networks", IEEE Trans. Computer-Aided Design, vol. 2, no. 3, pp. 202-211, March 1983.

[14] W.C. Elmore, "The Transient Response of Damped Linear Networks with particular regard to Wideband Amplifiers", Jl. Applied Physics, vol. 19, no. 1, pp. 55-63, January 1948.

(15) D. Zhou, S. Su, F. Tsui, D.S. Gao and J.S. Cong, "A Simplified Synthesis of Transmission Lines with a Tree Structure", Intl. Journal of Analog Integrated Circuits and Signal Processing, vol. 5, no. 1, pp. 19-30, January 1994. 
[16] R. Griffith, E. Chiprout, Q.J. Zhang and M.S. Nakhla, "A CAD Framework for Simulation and Optimization of High-Speed VLSI Interconnections", IEEE Trans. Circuits \& Systems, vol. 39, no. 11, pp. 893-906, Novembrr 1992.

[17] A.R. Djordjevic, R.F. Harrington and T.K. Sarkar, Matrix Parameters of Multiconductor Transmission Lines, Boston, MA: Artech House, 1989.

[18] E. Chiprout, "Moment-Matching Analysis of High Speed VLSI Interconnect Models", Doctoral Thesis, Carleton University, Ottawa, September 1994.

[19] R. Griffith and M.S. Nakhla, "Time-Domain Analysis of Lossy Coupled Transmission Lines", IEEE Trans. Microwave Theory \& Tech., vol. 38, no. 10, pp. 1480-1487, October 1990.

[20] L.T. Pillage and R.A. Rohrer, "Asymptotic Waveform Evaluation for Timing Analysis", IEEE Trans. Computer-Aided Design, vol. 9, no. 4, pp. 352-366, April 1990.

[21] J.E. Bracken, V. Raghavan and R.A. Rohrer, "Interconnect Simulation with Asymptotic Waveform Evaluation (AWE)", IEEE Trans. Circuits Systems-I, vol. 39, no. 11, pp. 869-878, November 1992.

[22] D. Xie and M.S. Nakhla, "Delay and Crosstalk Simulation of High Speed VLSI Interconnects with Nonlinear Terminations", IEEE Trans. Computer-Aided Design, vol. 12, no. 11, pp. 1798-1811, November 1993.

[23] E. Chiprout and M.S. Nakhla, "Analysis of Interconnect Networks using Complex Frequency Hopping", IEEE Trans. Computer-Aided Design, vol. 14, no. 2, pp. 186-200, February 1995. 
[24] R. Khazaka, E. Chiprout, M. Nakhla and Q.J. Zhang, "Analysis of High Speed Interconnects with Frequency Dependent Parameters", Proc. Intl. Symposium on Electromagnetic Compatibility, pp. 203-208, Zurich, Switzerland, March 1995.

[25] R. Achar, M.S. Nakhla and Q.J. Zhang, "Addressing High Frequency Issues in VLSI Interconnects with a Full-Wave Model and Complex Frequency Hopping", Proc. IEEE-ICCAD, San Jose, USA, November 1995.

[26] C.R. Paul, Analysis of Multiconductor Transmission Lines, John Wiley \& Sons, New York, USA, 1994.

[27] G.I. Costache, "Finite Element Method Applied to Skin-Effect Problems in Strip Transmission Lines", IEEE Trans. Microwave Theory and Tech., vol. 35, no. 11, pp. 1009-1013, November 1987.

[28] J. Poltz, “Optimizing VLSI Interconnect Model for SPICE Simulation", Jourual of Analog Integrated Circuits and Signal Processing, vol. 5, pp. 87-94, 1994.

[29] OptEM VLSI, version 2.0 Users' Manual, OptEM Engineering Inc., Calgary, Alberta, 1992.

[30] R.C. Booton, Computational Methods for Electromagnetics and Microwaves, John Wiley \& Sons, 1992.

[31] M.M. Ney, "Method of Moments as Applied to Electromagnetic Problems", IEEE Trans. Microwave Theory \& Tech., vol. 33, no. 10, pp. 972-980, October 1985.

[32] R.M. Biernacki, J.W. Bandler, J. Song and Q.J. Zhang, "Efficient Quadratic, Approximation for Statistical Design", IEEE Trans. Circuits Syst., vol. 36, no. 11, pp. 1449-1454, November 1989. 
[33] A.R. Djordjevic and T.K. Sarkar, "Closed-form Formulas for Frequency Dependent Resistance and Capacitance per unit length of Microstrip and Strip Transmission Lines", IEEE Trans. Microwave Theory Tech., vol. 42, no. 2, pp. 241-248, February 1994.

[34] S. Mitra, R.A. Rutenbar, L.R. Carley and D.J. Allstot, "A Methodology for Rapid Estimation of Substrate Coupled Switching Noise", Proc. Custom Integrated Circuits Conference, pp. 129-132, Santa Clara, USA, May 1995.

[35] L. Roy, S. Labonté and M. Li, "Microstrip Discontinuity Modeling for HighSpeed Interconnects", Proc. Canadian Conf. on Elec. \& Computer Engg., pp. 374-376, Montreal, Quebec, September 1995.

[36] M. Gilligan and S. Gupta, "A Methodology for Estimating Interconnect Capacitance for Signal Propagation Delay in VLSIs", Microelectronics Journal, vol. 26, no. 4, pp. 327-336, May 1995.

[37] U. Choudh'ry and A. Sangiovanni-Vincentelli, "Automatic Generation of Analytical Models for Interconnect Capacitances", IEEE Trans. Computer-Aided Design, vol. 14, no. 4, pp. 470-480, April 1995.

[38] R.A. Pucel, "Design considerations for monolithic microwave circuits", IEEE Trans. Microwave Theory \& Tech., vol. 29, no. 6, pp. 513-534, June 1981.

[39] R.H. Jansen, R.G. Arnold and I.G. Eddison, "A Comprehensive CAD Approach to the Design of MMICs up to MM-Wave Frequencies", IEEE Trans. Microwave Theory \&i Tech., vol. 36, no. 2, pp. 208-219, February 1988. 
[40] K.C. Gupta and T. Itoh (Guest Editors), "Special Issue on Computer-Aided Design", IEEE Trans. Microwave Theory \& Tech., vol. 36, no. 2, February 1988.

[41] S. D'Agostino and C. Paoloni, "Non-linear Analysis and Optimization of Monolithic Microwave Integrated Circuits", IEEE Trans. Microwave Theory \& Tech., vol. 43, no. 10, pp. 2504-2507, October 1995.

[42] S.J. Haefner, "Alternating Current of Rectangular Conductors", Proc. IRE, vol. 25, pp. 434-447, 1937.

[43] N.M. Nguyen and R.G. Meyer, "Si IC-compatible inductors and LC passive filters", IEEE Journal of Solid-State Circuits, vol. 27, no. 10, pp. 1028-1031, August 1990.

[44] P. Gray and R. Meyer, "Future Directions in Silicon ICs for RF Personal Communications", Proc. IEEE Custom Integrated Circuits Conference, pp. 83-90, Santa Clara, USA, May 1995.

[45] L.M. Burns, "Applications for GaAs and Silicon Integrated Circuits in Next Generation Wireless Communication Systems", IEEE Journal of Solid-State Circuits, vol. 30, no. 10, pp. 1088-1095, October 1995.

[46] M.W. Green, G.J. Green, R.G. Arnold, J.A. Jenkins and R.H. Jansen, “Miniature Multilayer Spiral Inductors for GaAs MMICs", Proc. GaAs IC Symposio -n pp. 303-306, 1989.

[47] E. Frlan, "Miniature Hybrid Microwave Integrated Circuit Passive Compnnent Analysis using Computer-Aided Design Techniques", Master of Engineering Thesis, Carleton University, Ottawa, August 1989. 
[48] E. Frlan, S. Meszaros, M. Cuhaci and J.S. Wight, "Computer-Aided Design of Square Spiral Transformers and Inductors", Proc. IEEE MTT.S Digest, pp. 661$664,1989$.

[49] D.Mi. Krafscik and D.E. Dawson, "A Closed-Form Expression Representing the Distributed Nature of the Spiral Inductor", Proc. IEEE, pp. 87-92, 1986.

[50] J.R. Long and M.A. Copeland, "Modeling of monolithic inductors and transformers for silicon RFIC design”, Proc. IEEE MTT-S Int. Symposium on Tech. for Wireless Applications, Vancouver, BC, pp. 140-141, February 1995.

[51] S. Chaki, S. Aono, N. Andoh, N. Tanino and O. Ishihara, "Experimental Study on Spiral Inductors”, IEEE MTT-S Digest, pp. 753-756, 1995.

[52] K.B. Ashby, W.C. Finley, J.J. Bastek, S. Moinian and I.A. Koullias, "High Q Inductors for Wireless Applications in a Complementary Silicon Bipolar Process", Proc. Bipolar \& BiCMOS Circuits \& Technology Meeting, pp. 179-182, Minneapolis, USA, 1994.

[53] E. Pettenpaul, H. Kapusta, A. Weisgerber, H. Mampe, J. Luginsland and I.

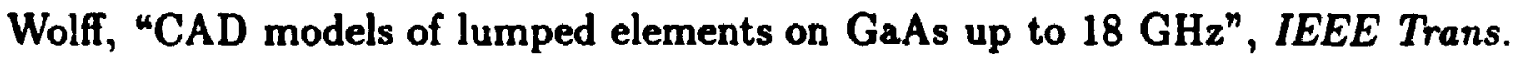
Microwave Theory \& Tech., vol. 36, no. 2, pp. 294-304, February 1988.

[54] HBT Design Manual version 1.00, Bell-Northern Research Ltd., Ottawa, Ontario, 1992.

[55] Microwave Spice Users' Guide, EESof Inc., 1988.

[56] W. McCulloch and W. Pitts, "A Logical Calculus of the Ideas Imminent in Nervous Activity", Bulletin of Mathematical Biophysics, 5:115-133, 1943. 
[57] B. Widrow and M Hoff, "Generalization and Information Storage in Networks of Adaline Neurons", in Self-Organizing Systems, M.C. Yovitz, G.T. Jacobi and G. Goldstein (editors), Spartan Books, Washington DC, USA, 1962.

[58] B.Widrow, "Adaline and Madaline - 1963", IEEE ICNN, 1963.

[59] M. Minksy and S. Papert, Perceptrons: An Introduction to Computational Geometry, The MIT Press, Cambridge, USA, 1969.

[60] P.J.W. Melsa, “Neural Networks: A Conceptual Overview”, Tellabs Research Center, Mishawaka, IN, USA TRC-89-08, August 1989.

[61] S. Haykin, Neural Networks: A Comprehensive Foundation, New York: IEEE Press, 1994.

[62] J.A. Freeman and D.M. Skapura, Neural Networks: Algorithms, Applicalions and Programming Techniques, Addison Wesley Publishing Company, Inc., 1991.

[63] P.J. Alsina and N.S. Gehlot, "Current Control of Induction Motors based on Input Voltage Output Current Models using Neural Networks", Proc. IECON, vol. 1, pp. 219-223, Maui, USA, November 1993.

[64] T.M. Martinetz, H.J. Ritter and K.J. Schulten, “Three dimensional Neural Net for learning visuometer coordination of a robot arm", IEEE Trans. Neural Networks, vol. 1, pp. 131-136 1989.

[65] T. Shibata and T. Fukuda, "Hierarchical Intelligent Control for Robotic Motion", IEEE Trans. Neural Networks, vol. 5, no. 5, pp. 823-832, September 1994. 
[66] D.Y. Tsai and N. Tanahashi, "Neural-Network based Boundary Detection of Liver Structure in CT Images for 3-D Visualization", Proc. IEEE Int. Conference on Neural Networks, pp. 3484-3489, Orlando, USA, June 1994.

[67] K.H. Lee, "The Difference Method: A Way of Detecting Abnormalities in MRI Images", Proc. Intl. Joint Conference on Neural Networks, vol. 2, pp. 1227-1230, Nagoya, Japan, October 1993.

[68] T. Conde, "Automatic Neural Detection of Anomalous Electrocardiogram (ECG) Signals", Proc. IEEE Int. Conference on Neural Networks, pp. 3552-3558, Orlando, USA, June 1994.

[69] K. Kondo, H. Kamata and Y. Ishida, "Speaker Independent Spoken Digits Recognition using LVQ", Proc. IEEE Int. Conference on Neural Networks, pp. 44484451, Orlando, USA, June 1994.

[70] S.H. Huang and H.C. Zhang, "Artificial Neural Networks in Manufacturing: Concepts, Applications and Perspectives", IEEE Trans. Comp., Pack., \& Manufacturing Tech., Part A, vol. 17, no. 2, pp. 212-228, June 1994.

[71] R. Uhrig, L. Tsoukalas and A. Ikonomopoulos, "Application of Neural Networks and Fuzzy Systems to Power Plants", Proc. IEEE Int. Conference on Neural Networks, pp. 3703-3718, Orlando, USA, June 1994.

[72] Y. Li, R.L. Mahajan and J. Tong, "Design Factors and their Effect on PCB Assembly, Yield-Statistical and Neural network Predictive Models", IEEE Trans. Comp., Pack. and Manufacturing Tech., Part A, vol. 17, no.2, Pp. 183-191, June 1994. 
[73] B. Kim and G.S. May, "Modeling Reactive Ion Etching of Silicon Dioxide Films using Neural Networks", Proc. 4th Electronic Comp. \& Tech. Conference, pp. 273-278, 1994.

[74] M.D. Baker, C.D. Himmel and G.S. May, "Time Series Modeling of Reactive Ion Etching Using Neural Networks", IEEE Trans. Semiconductor Manufacturing, vol. 8, no. 1, pp. 62-71, February 1995.

[75] B. Dolenko, H. Card, M. Neumann and E. Shwedyk, "Classifying Cereal Grains using Backpropagation and Cascade Correlation Networks", Canadian Journal of Elec. \& Comp. Engg., vol. 20, no. 2, pp. 91-95, April 1995.

[76] C.L. Wilson, "Self-Organizing Neural Network System for Trading Common Stocks", Proc. IEEE Int. Conference on Neural Networks, pp. 3651-3654, Orlando, USA, June 1994.

[77] T. Falas, A. Charitou and C. Caralambous, "The Application of Artificial Neural Networks in the Prediction of Earnings", Proc. IEEE Int. Conference on Neural Networks, pp. 3629-3633, Orlando, USA, June 1994.

[78] B.A. Telfer, H.H. Szu, P. Rennert and C. Rumpel, "Neural Network Prediction of Mortality", Proc. Intl. Joint Conference on Neural Networks, vol. 1, pp. 963-966, Nagoya, Japan, October 1993.

[79] S.S. Rangwala and G.L. Goodwin, "An Application of Neural Networks to Prediction of SL Laser Package Coupling Stability Characteristics", Proc. 49rd Elcctronic Comp. \& Tech. Conference, pp. 169-174, Orlando, USA, June 1993. 
[80] B.G. Hoskins and M.R. Haskard, "Artificial Neural Network Techniques for the Estimation of Thick film Resistors", Microelectronics Journal, vol. 26, no. 1, pp. 9-16, January 1995.

[81] S.S. Han, M. Ceiler, S.A. Bidstrup and P. Kohli, "Modeling the Properties of PECVD Silicon Dioxide Films using Optimized Backpropagation Neural Networks", IEEE Trans. Comp., Pack. and Manufacturing Tech., Part A, vol. 17, no.2, pp. 174-182, June 1994.

[82] R.S. Collica, J.P. Card and W. Martin, "SRAM Bitmap Shape Recognition using Neural Networks", IEEE Trans. Semiconductor Manufacturing, vol. 8, no. 3, pp. 326-332, August 1995.

[83] J.D. Kant and J.L. Drezen, "Electromagnetic Field Parallel Computation with Hopfield Neural Network", IEEE Transactions on Magnetics, vol. 31, no. 3, pp. 1968-1971, May 1995.

[84] M. Vai, S. Prasad and H. Wong, "A Smith Chart represented by a Neural Network and its Applications", IEEE MTT-S Intl. Microwave Symp. Digest, pp. 1565-1568, Albequerque, USA, August 1992.

[85] M. Vai and S. Prasad, "Automatic Impedance Matching with a Neural Network", IEEE Microwere \& Guided Wave Letters, vol. 3, no. 10, pp. 353-354, October 1993.

[86] S. Ratnajeevan, H. Hoole and M.K. Haldar, "Optimization of Electromagnetic Devices: Circuit Models, Neural Networks and Gradient Methods in Concert”, IEEE Transactions on Magnetics, vol. 31, no. 3, pp. 2016-2019, May 1995. 
[87] Q.J. Zhang and M.S. Nakhla, "Signal Integrity Analysis and Optimization of VLSI Interconnects Using Neural Network Models", Proc. IEEE International Symp. Circuits \& Systems, pp. 459-462, London, UK, May 1994.

[88] A. Veluswami, Q.J. Zhang and M.S. Nakhla, "A Neural Network Model for Propagation Delays in Systems with High Speed VLSI Interconnect Networks", Proc. Custom Integrated Circuits Conference, pp. 387-390, Santa Clara, USA, May 1995.

[89] A. Veluswami, A.H. Zaabab, Q.J. Zhang and M.S. Nakhla, "The Application of Neural Networks to High Speed Interconnect Simulation", Proc. Canadian Conference on Electrical and Computer Engineering, pp. 381-384, Montreal, Quebec, September 1995.

[90] A. H. Zaabab, Q.J. Zhang and M.S. Nakhla, "A Neural Network Approach to Circuit Optimization And Statistical Design", IEEE Trans. Microwave 6 Theory Tech., vol. 43, no. 6, pp. 1349-1358, June 1995.

[91] G. Kothapalli, "Artificial Neural Networks as Aids in Circuit Design", Microelectronics Journal, vol. 26, no. 6, pp. 569-578, September 1995.

[92] P. Ojala, J. Saarinen, P. Elo and K. Kaski, "Novel Technology Independent Neural Network Approach on Device Modeling Interface”, IEE Proceedings - Circuits, Devices \& Systems, vol. 142, no. 1, pp. 74-82, February 1995.

[93] SALI: Structure Analysis for Lossy Interconnect, Northern Telecom Ltd., Ottawa, Ontario, 1995. 
[94] F. Halary, Graph Theory, Shanghai Science and Technology Publishing Company, 1980.

[95] Matlab Version 4.2, The Mathworks, Inc., Natick, USA, 1994.

[96] I. Bahl and P. Bhartia, Microwave Solid State Circuit Design, New York, USA: Wiley, 1988. 


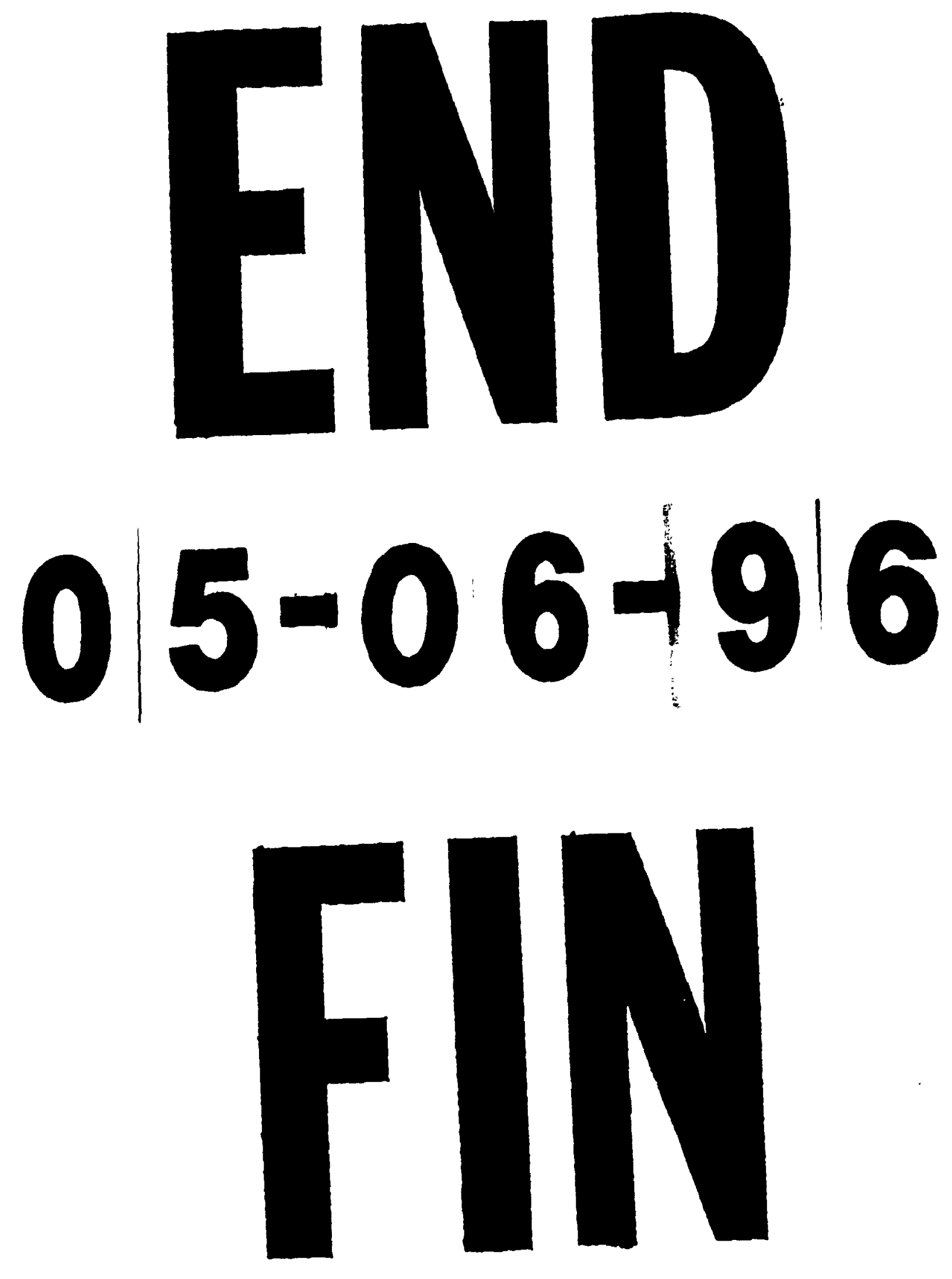

